\title{
WestVirginiaUniversity
}

THE RESEARCH REPOSITORY @ WVU

Graduate Theses, Dissertations, and Problem Reports

2004

\section{Multispectral segmentation of whole-brain MRI}

Nitya Krishnan

West Virginia University

Follow this and additional works at: https://researchrepository.wvu.edu/etd

\section{Recommended Citation}

Krishnan, Nitya, "Multispectral segmentation of whole-brain MRI" (2004). Graduate Theses, Dissertations, and Problem Reports. 1548.

https://researchrepository.wvu.edu/etd/1548

This Thesis is protected by copyright and/or related rights. It has been brought to you by the The Research Repository @ WVU with permission from the rights-holder(s). You are free to use this Thesis in any way that is permitted by the copyright and related rights legislation that applies to your use. For other uses you must obtain permission from the rights-holder(s) directly, unless additional rights are indicated by a Creative Commons license in the record and/ or on the work itself. This Thesis has been accepted for inclusion in WVU Graduate Theses, Dissertations, and Problem Reports collection by an authorized administrator of The Research Repository @ WVU. For more information, please contact researchrepository@mail.wvu.edu. 
Multispectral Segmentation of Whole Brain MRI

\title{
Nitya Krishnan
}

Thesis submitted to the

College of Engineering and Mineral Resources

at West Virginia University

in partial fulfillment of the requirements

for the degree of

\section{Master of Science}

In

Electrical Engineering

\author{
Dr. Susan K Lemieux, Ph.D., Co-Chair \\ Dr. Mark Jerabek, Ph.D., Co-Chair \\ Dr Wils Cooley, Ph.D. \\ Dr. Aina Puce, Ph.D.
}

Dr. Donald Adjeroh, Ph.D.

Lane Department of Computer Science and Electrical Engineering

Center for Advanced Imaging, WVU School of Medicine

\section{Morgantown, West Virginia}

2004

Keywords: MRI, Fuzzy c-means clustering, image segmentation, tissue types 


\section{ABSTRACT \\ Multispectral Segmentation of Whole Brain MRI}

Nitya Krishnan

Magnetic Resonance Imaging (MRI) is a widely used medical technology for diagnosis and detection of various tissue abnormalities, tumor detection, and in evaluation of either residual or recurrent tumors. This thesis work exploits MRI information acquired on brain tumor structure and physiological properties and uses a novel image segmentation technique to better delineate tissue differences.

MR image segmentation will be important in distinguishing between boundaries of different tissues in the brain. A segmentation software tool was developed that combines the different types of clinical MR images and presents them as a single colored image. This technique is based on the fuzzy c - means (FCM) clustering algorithm. The MR data sets are used to form five-dimensional feature vectors. These vectors are segmented by FCM into six tissue classes for normal brains and nine tissue classes for human brains with tumors. The segmented images are then compared with segmentation performed using Statistical Parametric Mapping (SPM2)-software that is commonly used for brain tissue segmentation. The results from segmenting the whole volume MRI using FCM show better distinction between tumor tissues than SPM2. 


\section{ACKNOWLEDGEMENTS}

I would like to wholeheartedly thank my advisor Dr. Susan Lemieux for her continued support, guidance and encouragement during the course of this research work. Special thanks to Dr. Aina Puce for her help and valuable thoughts during my research work.

I also wish to thank Dr. Mark Jerabek, Dr. Wils Cooley, Dr. Donald Adjeroh my committee members, for their advice and support. Also special thanks to Dr. Raymond Raylman for his constant encouragement and support in carrying out the experiments.

I would also like to thank Dr. Mark Haut, Dr. Gary Marano, and Dr. Stephen Bloomfield who helped in recruiting subjects for this research work. Thanks to Dr. Michael Parsons, Kamal Sheikh, Dr. Jim Thompson, and Dr. Svenja Lowitzsch for their help in data analysis.

And finally I would like to thank the members of Center for Advanced Imaging / Department of Radiology for all their help and guidance. 


\section{Dedications}

To my parents and my brother for their support and guidance throughout my life and their help to fulfill my dreams in pursuing my masters.

To Susan, my research professor who has been like my mother in this country and continuously encouraged and guided me both professionally and personally.

And above all, to my beloved husband Bharath, who is my soul-mate, inspiration and friend. 


\section{Table of Contents}

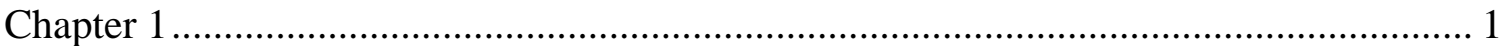

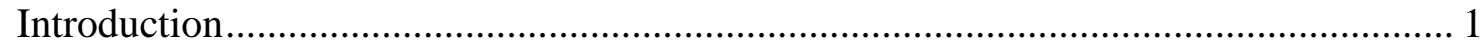

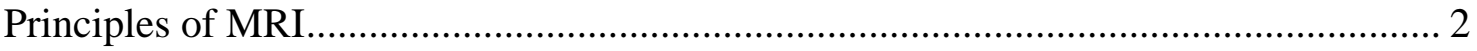

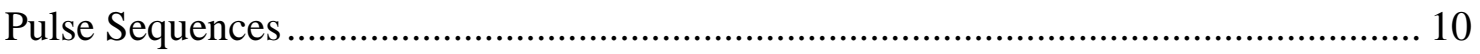

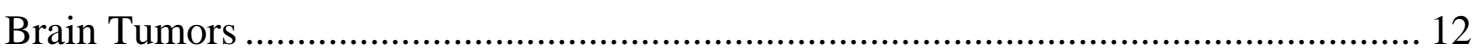

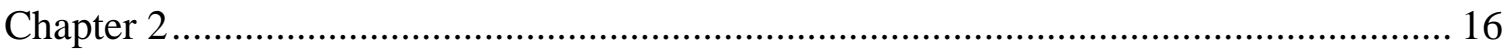

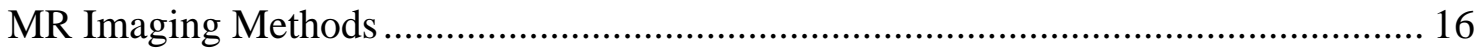

Fast Fluid - Attenuated Inversion-Recovery (FLAIR)…………………………...... 16

Diffusion - Weighted Imaging (DWI).................................................................. 17

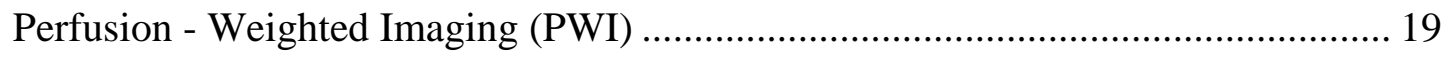

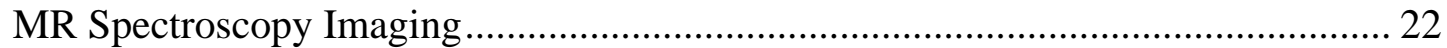

Segmentation Using Fuzzy C - Means Clustering Algorithm ....................................... 24

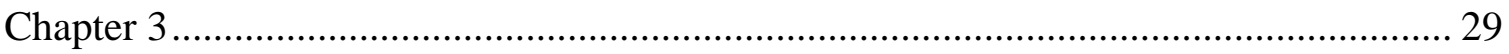

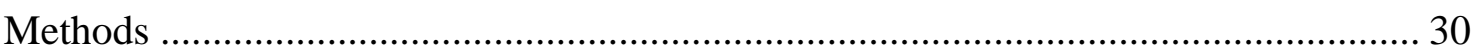

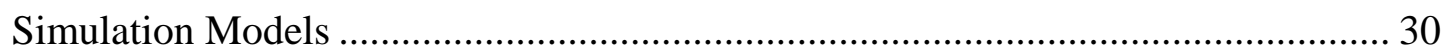

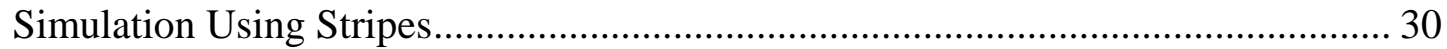

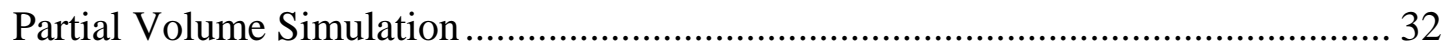

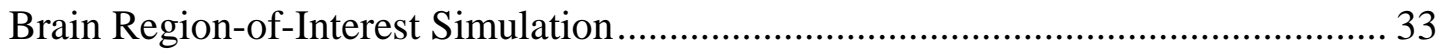

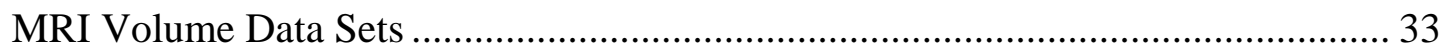

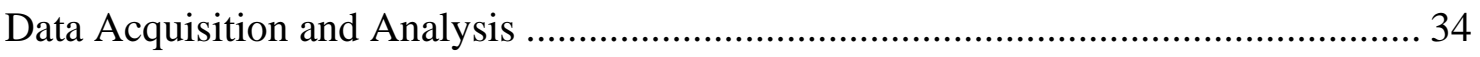

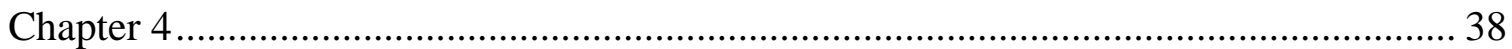

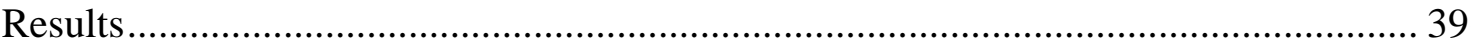

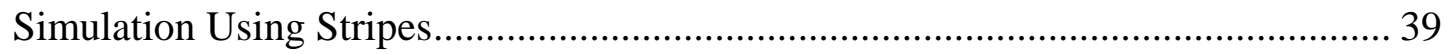

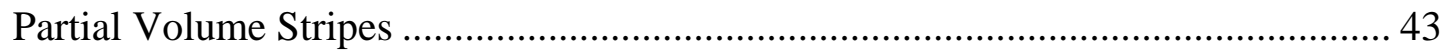

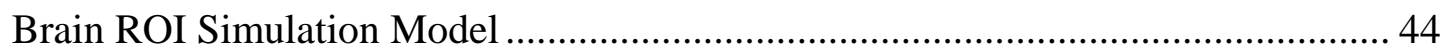

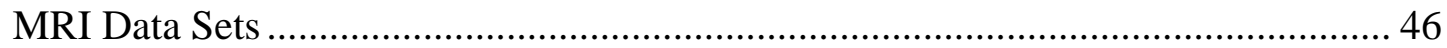

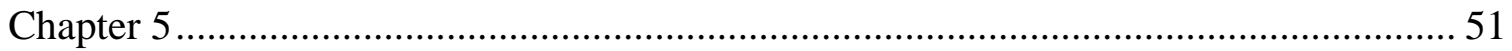

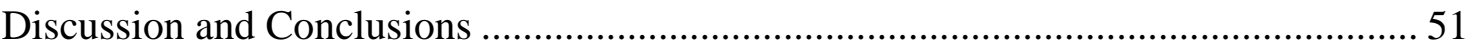

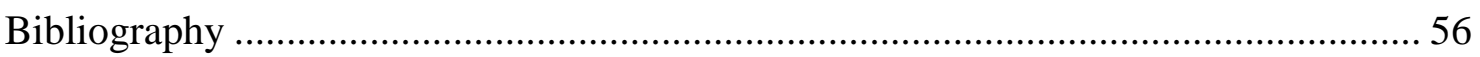

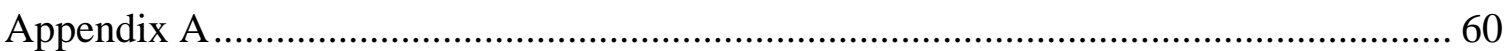




\section{Table of Figures}

Figure 1.1 Proton alignment in the absence of magnetic field. ....................................... 2

Figure 1.2 Protons in a magnetic field aligned along external magnetic field. .................. 3

Figure 1.3 Interaction of a proton with magnetic field................................................. 5

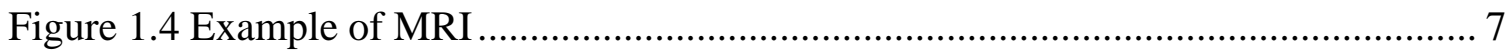

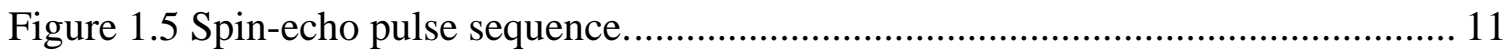

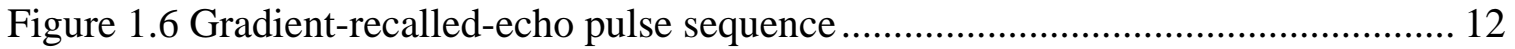

Figure 2.1 Diffusion-weighted imaging pulse sequence. .............................................. 18

Figure 2.2 Methodology for perfusion imaging.......................................................... 21

Figure 2.3 Spectroscopic image of tumor patient with voxel at normal tissue................. 24

Figure 2.4 Spectroscopic image of tumor patient with voxel at tumor tissue ................. 24

Figure 2.5 3D Cluster center plot with four classes of a simulation dataset. .................. 28

Figure 2.6 Membership functions with respect to $\mathrm{m}$.................................................. 29

Figure 2.7 Membership function indicating threshold value ...................................... 29

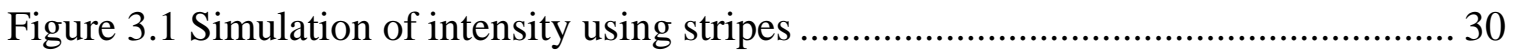

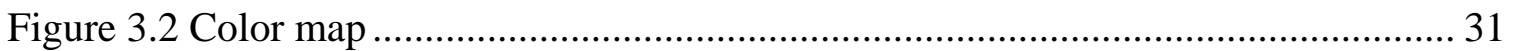

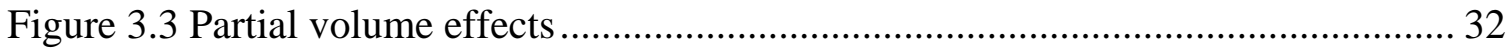

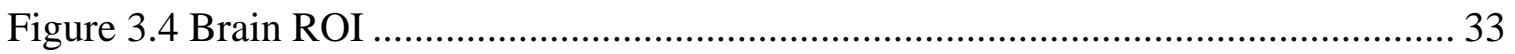

Figure 3.5 Sketch of the central portion of the midline of the brain showing anatomical

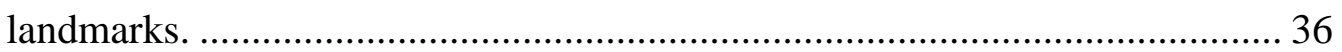

Figure 4.1 Simulation stripes of seven tissues with three types of images..................... 39

Figure 4.2 Color map used for normal subjects ...................................................... 39

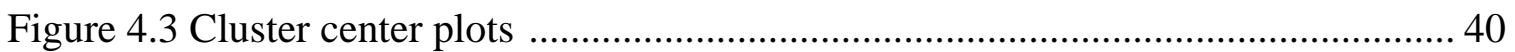

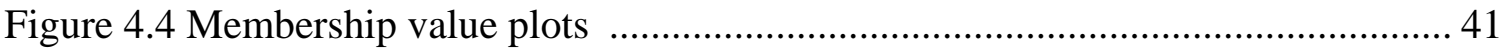

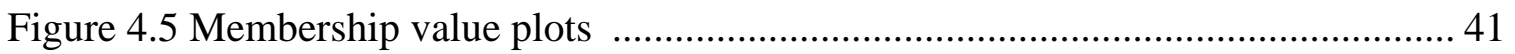

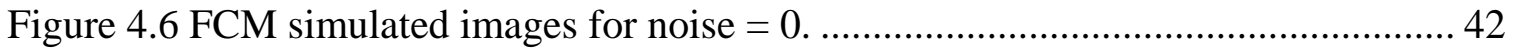

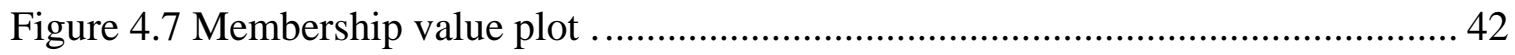

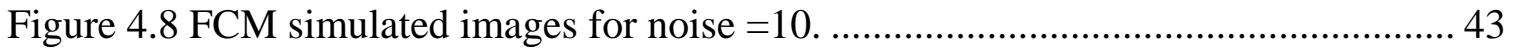

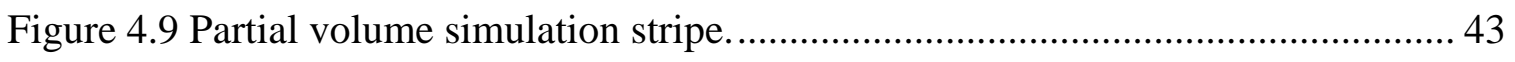

Figure 4.10 FCM segmented images for partial volume simulation. ............................. 44

Figure 4.11 Brain ROI of three image vectors......................................................... 45

Figure 4.12 FCM segmented Brain ROI.................................................................... 45

Figure 4.13 FCM segmentation of normal subject. ....................................................... 46

Figure 4.14 Difference maps of normal subject...................................................... 47

Figure 4.15 FCM segmentation of tumor subject .................................................... 48

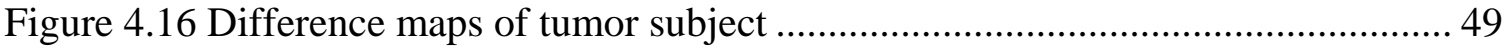




\section{List of Tables}

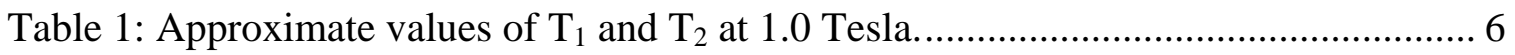

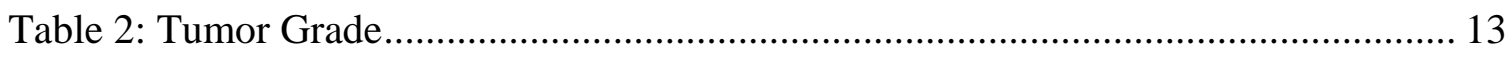

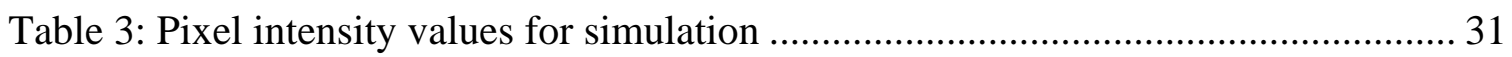

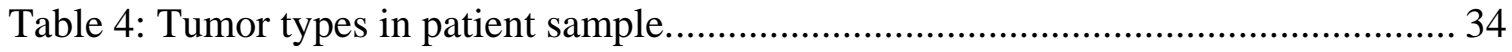

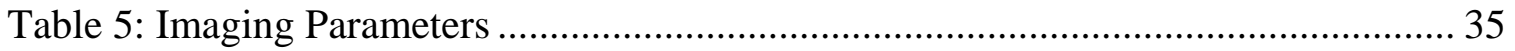

Table 6: Quantitative Difference in GM, WM, CSF maps using four feature vectors..... 50

Table 7: Quantitative Difference in GM, WM, CSF maps using five feature vectors. .... 50 


\section{Chapter 1}

\section{Introduction}

Magnetic resonance imaging (MRI) is used as a medical diagnostic tool for studying the human anatomy. MRI is based on the principles of nuclear magnetic resonance (NMR), a technique that provides information about properties of materials. NMR was developed by Bloch and Purcell in the 1940’s. In the 1970's Paul Lauterbur, Ray Damadian, and Peter Mansfield began to use the principles of NMR in MRI as an imaging modality in the head, spine and body. MRI produces images of high spatial resolution (mm) with good soft tissue contrast that has made it useful for detection of diseases. In 2003, Paul Lauterbur and Peter Mansfield were awarded the Nobel Prize in Physiology or Medicine for their simultaneous pioneering research using magnetic resonance to image the human body.

The focus of this thesis is the use of MRI in brain tumors utilizing their physiological properties and using image segmentation techniques to obtain better delineation. MRI is a commonly used modality for detection of brain tumor, their spread and in evaluating residual or recurrent brain tumors. The growth in technology allows for improved tumor detection and biological characterization of the tumor. Brain tumor detection is an important application which continues to be of keen interest to clinicians. MR imaging is utilized for tumor biopsy planning, treatment monitoring, and long term monitoring for reoccurrence.

Current clinical methods that are used to differentiate tumors from normal tissues, even after the injection of a contrast medium, may not detect all tumors and sometimes tumors may not enhance well. Hence, there is a need to combine the various MRI data sets to classify brain tissues and differentiate them from abnormal tumor tissues. In particular, current brain segmentation methods like Statistical Parametric Mapping (SPM2) fail for brain images of patients with brain lesions. This is due to the fact that SPM uses a priori

probability images for segmentation and in the case of patient data there is no valid $a$ 
priori model. In this thesis, a software tool in MATLAB has been developed for segmenting whole volume MRI data sets using the Fuzzy c-means (FCM) clustering algorithm.

\section{Principles of MRI}

Nuclei with odd number of protons and neutrons have a non-zero nuclear magnetic moment. The hydrogen nucleus, composed of a single proton, has the largest magnetic moment and is abundantly found in the body tissues (since the human body mostly consists of water, $1 \mathrm{~mm}^{3}$ of water $=6.7 \times 10^{19}$ hydrogen protons). Hence, the hydrogen proton is the primary nucleus used for MRI because it produces the strongest signal. The proton precessing in the absence of an external magnetic field may be oriented along any direction as shown in Figure 1.1.

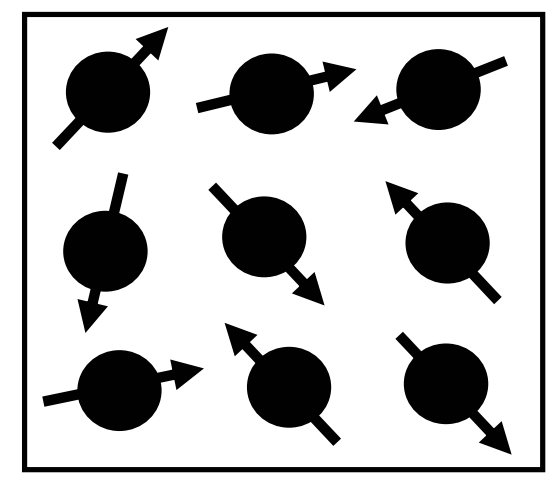

Figure 1.1 Proton alignment in the absence of magnetic field ${ }^{1}$.

The net magnetic moment per unit volume known as the magnetization vector is given by:

$$
\mathbf{M}=\mathbf{M}_{\mathrm{x}} \mathbf{i}+\mathbf{M}_{\mathrm{y}} \mathbf{j}+\mathbf{M}_{\mathrm{z}} \mathbf{k}
$$

Where $\mathbf{M}$ - magnetization vector in the $\mathrm{x}, \mathrm{y}, \mathrm{z}$ direction, $\mathbf{i}, \mathbf{j}, \mathbf{k}$ are unit vectors.

In the absence of an external magnetic field, the net magnetization vector $\mathbf{M}$ will be zero. When placed in a strong external magnetic field $\mathrm{B}_{0}$, the magnetic moments of the proton align (orient) themselves along the magnetic flux lines and precess about the flux lines. The magnetic moments of the protons precess along the z-direction (in the direction of $\mathrm{B}_{0}$ ) as shown in Figure 1.2. 


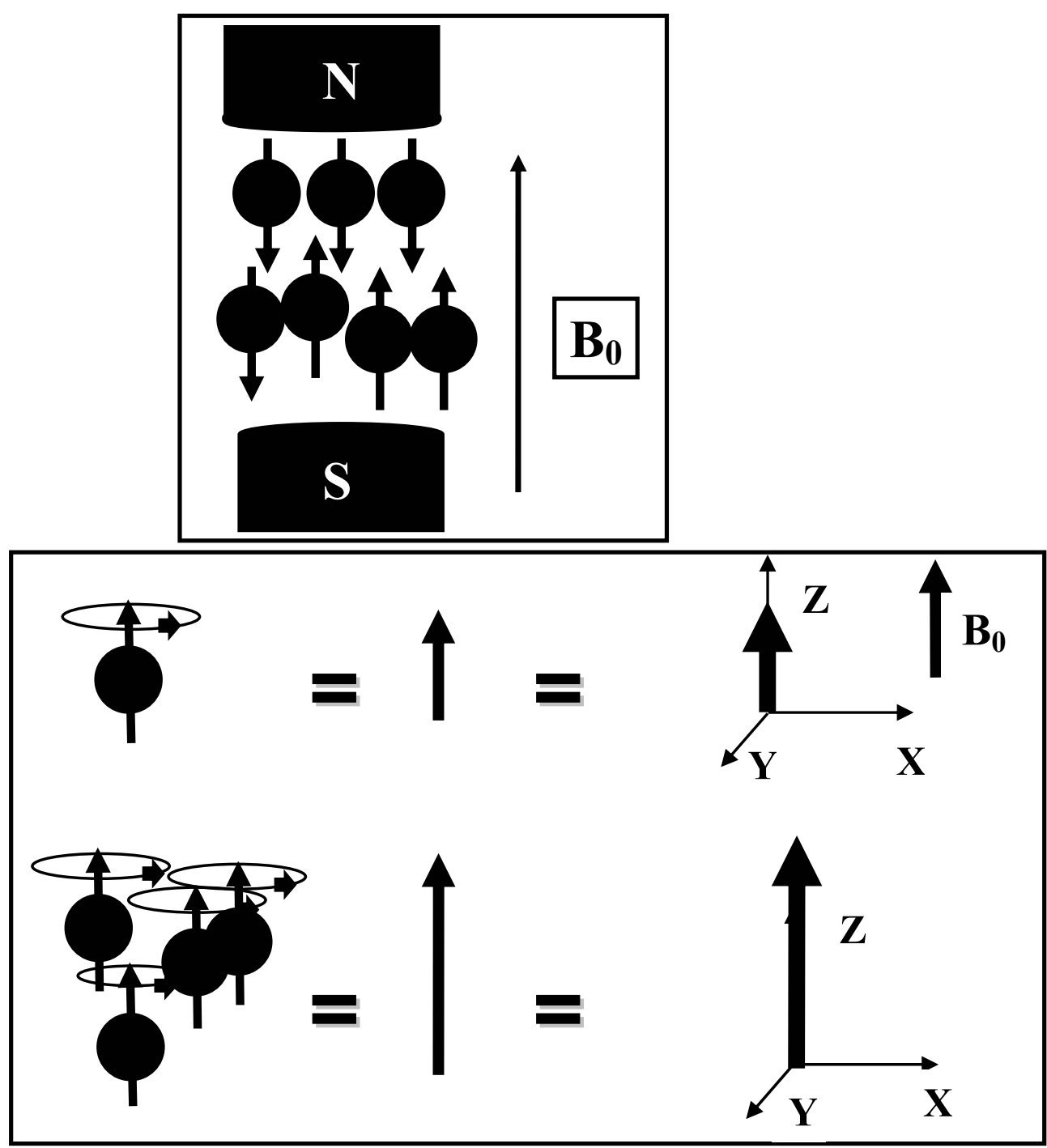

Figure 1.2 Protons in a magnetic field aligned along external magnetic field $\mathrm{B}_{0}{ }^{1}$.

The equilibrium value of the magnitude of proton magnetization $\mathrm{M}_{0}$, (average of all individual nuclear magnetic dipole moments), in the presence of magnetic field $\mathrm{B}_{0}$ is given as:

$$
\mathrm{M}_{0}=\frac{\mathrm{N} \gamma^{2} \mathrm{~h}^{2} \mathrm{I}(\mathrm{I}+1) \mathrm{B}_{0}}{3 \mathrm{kT} \mathrm{T}_{\mathrm{s}}}
$$

Where,

$\mathrm{B}_{0}$ - Static magnetic field,

$\mathrm{N}$ - Number of proton spins per unit volume, 
$\gamma$ - Gyromagnetic ratio, a constant unique for each nucleus,

h - Planck's constant,

I - Proton spin,

$\mathrm{T}_{\mathrm{s}}$ - Absolute sample temperature in Kelvin,

k - Boltzmann's constant.

Thus magnetization $\mathrm{M}_{0}$ is proportional to external magnetic field $\mathrm{B}_{0}$. The magnetic moments exhibit the property of precessing around the field $\mathrm{B}_{0}$. The frequency of precession is given by the Larmor frequency:

$$
\mathrm{f}_{\mathrm{L}}=\frac{\gamma \mathrm{B}_{0}}{2 \pi}
$$

For the proton, $\frac{\gamma}{2 \pi}=42.58 \mathrm{MHz} /$ Tesla, thus the Larmor frequency will be in radiofrequency region (40-50 MHz). The Larmor frequency is proportional to the magnetic field strength $\mathrm{B}_{0}$ from Equation 1.3.

To obtain an MR signal, radiofrequency (RF) pulses of electromagnetic radiation $\mathrm{B}_{1}$ are applied at the Larmor frequency $\mathrm{f}_{\mathrm{L}}$ perpendicular to the main magnetic field, $\mathrm{B}_{0}$ disturbing the magnetic moments of the protons from their equilibrium position ${ }^{2}$. The protons are aligned along the z-axis in equilibrium with the longitudinal magnetization $\left(\mathbf{M}_{z}\right)$ of magnitude $\mathbf{M}_{0}$. This alignment, when disturbed by a 90 degree RF pulse displaces $\mathbf{M}_{\mathrm{z}}$ to $\mathbf{M}_{\mathrm{xy}}$. The total displacement is proportional to the RF-pulse energy and also the Larmor frequency. If the energy of the RF pulse is sufficient to tip the magnetization vector by 90 degrees (tip angle), then $\mathbf{M}_{\mathrm{z}}$ is tipped into the transverse plane $(\mathrm{x}, \mathrm{y})$. The magnetization vector continues to precess about $\mathrm{B}_{0}$ in the transverse plane. This time-varying magnetization induces flux changes which are detected in a RF coil. The interaction of a proton with the magnetic field is shown in Figure 1.3. 


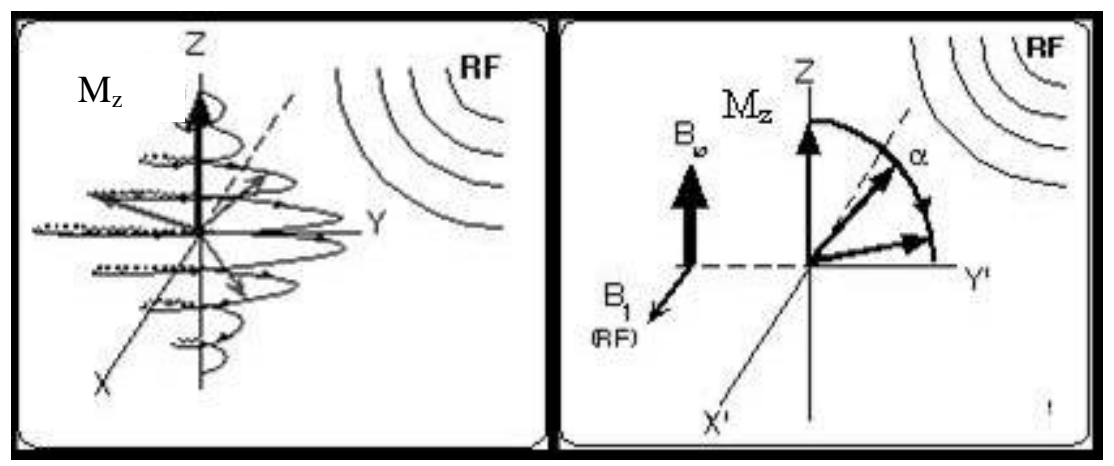

Figure 1.3 Interaction of a proton with magnetic field - a) An RF-pulse creates the opposed field $\mathrm{B}_{1}$, displacing $\mathbf{M}_{\mathrm{z}}$ and causing the precession about the z-axis. b) A large RF-pulse displaces $\mathbf{M}_{\mathrm{z}}$ into the transverse plane (z-component is zero; $\mathrm{x}, \mathrm{y}$ components are maximum) ${ }^{3}$.

The protons when returning to their equilibrium position along the z-axis undergo relaxation processes which can be described in two ways: The relaxation component of the net magnetization vector along the z-axis is called longitudinal or spin-lattice relaxation and this time constant is called $T_{1}$. The relaxation component of net magnetization vector in the xy plane is called transverse or spin-spin relaxation and this time constant is called $\mathrm{T}_{2}$.

\section{Relaxation constants}

After a 90 degree RF pulse excitation, the return of the longitudinal component of magnetization to its equilibrium state along the z-direction is termed the longitudinal or spin-lattice relaxation. The $T_{1}$ relaxation is due to the loss of energy by the protons to the surrounding nuclei as they return to their equilibrium state. The $\mathrm{T}_{1}$ relaxation is approximately an exponential process and is specified in milliseconds. $\mathrm{T}_{2}$ relaxation is the decay of transverse magnetization, also an exponential process, represents the overall loss of phase coherence due to dephasing of the spins. Each proton has unique $T_{1}$ and $T_{2}$ time constants depending on the surrounding environment. The $T_{2}$ time constant is typically about 10-1000 milliseconds long. The $\mathrm{T}_{2}$ spin dephasing can also be caused by tissue inhomogeneities, field inhomogeneities, or tissue interfaces. These inhomogeneities further reduce the relaxation constant and $T_{2}$ is called the effective $T_{2}$ or 
$\mathrm{T}_{2}{ }^{*}$ time constant. The $\mathrm{T}_{2}{ }^{*}$ time constant is much shorter than $\mathrm{T}_{2}$. The relaxation constants are important parameters of MR and are different for various brain tissues as shown in Table 1.

Table 1: Approximate values of $\mathrm{T}_{1}$ and $\mathrm{T}_{2}$ at $1.0 \mathrm{Tesla}^{4}$.

\begin{tabular}{|c|c|c|}
\hline Tissue & $\mathbf{T}_{\mathbf{1}}(\mathbf{m s e c})$ & $\mathbf{T}_{\mathbf{2}}(\mathbf{m s e c})$ \\
\hline White matter & 390 & 90 \\
\hline Gray matter & 520 & 100 \\
\hline Cerebrospinal Fluid (CSF) & 2000 & 300 \\
\hline Muscle & 600 & 40 \\
\hline Fat & 180 & 90 \\
\hline Liver & 270 & 50 \\
\hline Blood & 800 & 180 \\
\hline
\end{tabular}

\section{Image Contrast}

In MR images, the magnitude of the MR signal is represented by the intensity of each pixel in the image. Contrast in the images depends on the pulse sequences used, and also on the tissue characteristics. The type of image produced depends on the TR and TE values. TR is the repetition time of the 90 degree pulse and TE is the echo time, the amount of time between the RF excitation and the measurement of the echo. The relaxation constants $T_{1}$ and $T_{2}$ provide good sources of contrast and by varying TR and TE the desired contrast can be obtained as shown in Figure 1.4. Varying these parameters along with different sequences allow different tissue types and pathologies to be highlighted. Radiologists, trained to interpret MRI scans, use these varied contrast images to detect abnormalities. A $\mathrm{T}_{1}$-weighted image is characterized by short $\mathrm{TR}$ and short TE values. A $\mathrm{T}_{2}$-weighted image has a long TR and a long TE value. A proton density image has a long TR and a short TE value. Image contrast can also be changed by the exogenous contrast agents that have paramagnetic properties like gadoliniumdiethylene triamine penta-acetic acid dimeglumine (Gd-DTPA). Shown in Figure 1.4 are $\mathrm{T}_{1}$-weighted and $\mathrm{T}_{2}$-weighted images taken by a $3 \mathrm{~T}$ scanner. 

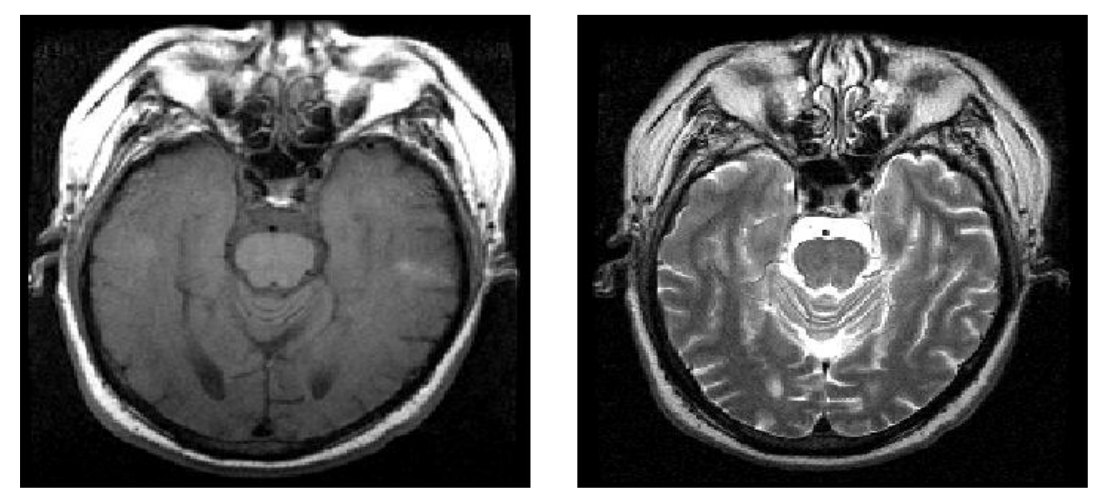

Figure 1.4 Example of MRI a) $\mathrm{T}_{1}$-weighted image b) $\mathrm{T}_{2}$-weighted image

\section{Bloch Equation}

During relaxation, the protons trace a spiral motion which can be described by the Bloch equation $^{5}$. The equation of motion of magnetization around the total magnetic field present is given by the Bloch equation as:

$$
\frac{d \mathbf{M}}{d t}=\gamma \mathbf{M} \times \mathbf{B}
$$

Where:

B - Total magnetic field

M - Magnetization vector

$\gamma$ - Gyromagnetic ratio

The Bloch equation states that the time rate of change of $\mathbf{M}$ is proportional to the cross product of $\mathbf{M}$ with $\gamma \mathbf{B}$. Hence if $\mathbf{B}$ is static, this motion corresponds to a precession of $\mathbf{M}$ about $\mathbf{B}$ at an angular frequency of $\gamma\|\mathbf{B}\|$. If $\mathbf{B}$ is time varying, then the precessional frequency also becomes time varying. The Bloch equation can be written to include the relaxation constants $T_{1}$ and $T_{2}$ and is given by:

$$
\frac{d \mathbf{M}}{d t}=\mathbf{M} \times \gamma \mathbf{B}-\frac{\left(\mathbf{M}_{\mathbf{x}} \mathbf{i}+\mathbf{M}_{\mathbf{y}} \mathbf{j}\right)}{\mathrm{T}_{2}}-\frac{\left(\mathbf{M}_{\mathbf{z}}-\mathbf{M}_{0}\right) \mathbf{k}}{\mathrm{T}_{1}}
$$

Where:

B - Total magnetic field

M - Magnetization vector 
$\gamma$ - Gyromagnetic ratio

$\mathrm{T}_{1}$ - spin lattice (longitudinal $-\mathrm{z}$ ) relaxation time constant

$\mathrm{T}_{2}$ - spin-spin (transverse-xy) relaxation time constant

$\mathrm{M}_{0}$ - equilibrium magnetization due to $\mathrm{B}_{0}$

$\mathbf{M}_{\mathbf{x}}, \mathbf{M}_{\mathbf{y}}, \mathbf{M}_{\mathbf{z}}$ - magnetization in the x, y, z plane respectively

$\mathbf{i}, \mathbf{j}, \mathbf{k}$ - unit vectors in $\mathrm{x}, \mathrm{y}, \mathrm{z}$ directions respectively.

The solution to the modified Bloch equation is shown below:

$$
\begin{aligned}
& \mathbf{M}_{\mathbf{z}}(\mathrm{t})=\mathrm{M}_{0}\left(1-\mathrm{e}^{-\mathrm{t} / \mathrm{T}_{1}}\right) \\
& \mathbf{M}_{\mathbf{x}}(\mathrm{t})=\mathbf{M}_{\mathbf{x y}} \cos \omega_{0} \mathrm{t}\left(\mathrm{e}^{-\mathrm{t} / \mathrm{T}_{2}}\right) \\
& \mathbf{M}_{\mathbf{y}}(\mathrm{t})=-\mathbf{M}_{\mathbf{x y}} \sin \omega_{0} \mathrm{t}\left(\mathrm{e}^{-\mathrm{t} / \mathrm{T}_{2}}\right)
\end{aligned}
$$

\section{Signal Measurement}

After the application of the RF pulses, emissions by the protons of RF radiation by the relaxation process at the resonant frequency take place. Flux changes are detected in the receiver coil and the precessing magnetization in the transverse direction gives rise to an electromotive force $\in$ which is given as the rate of change of flux $(\varphi)$ in the coil.

$$
\in=-\frac{\partial \varphi}{\partial \mathrm{t}}
$$

If $\mathbf{B}_{\mathbf{1}}(\mathrm{x}, \mathrm{y}, \mathrm{z})$ is the magnetic field at a point $(\mathrm{x}, \mathrm{y}, \mathrm{z})$, the EMF in the coil is given as:

$$
\mathrm{d} \in=-\frac{\partial}{\partial \mathrm{t}}\left\{\mathbf{B}_{\mathbf{1}}(\mathrm{x}, \mathrm{y}, \mathrm{z}) \bullet \mathbf{M}(\mathrm{x}, \mathrm{y}, \mathrm{z}, \mathrm{t})\right\} \mathrm{d} \mathbf{r}
$$

Thus the total EMF signal $\mathrm{s}(\mathrm{t})$ is given as:

$$
\int_{\text {vol }} \mathrm{d} \in=\mathrm{s}(\mathrm{t})=-\int_{\text {vol }} \frac{\partial}{\partial \mathrm{t}}\left\{\mathbf{B}_{\mathbf{1}}(\mathrm{x}, \mathrm{y}, \mathrm{z}) \bullet \mathbf{M}(\mathrm{x}, \mathrm{y}, \mathrm{z}, \mathrm{t})\right\} \mathrm{d} \mathbf{r}
$$

The voltage is then amplified and demodulated to provide the MR signal. 


\section{Signal Localization using Gradients}

Within the magnet, gradient coils are placed so that they superimpose their fields on the main magnetic field $\mathrm{B}_{0}$. A set of gradient coils provide varying magnetic field strengths along the $x\left(G_{x}\right)$, y $\left(G_{y}\right)$ and $z\left(G_{z}\right)$ direction. Gradients change only the spatial variation of the amplitude of $\mathrm{B}_{0}$ and not the direction of $\mathrm{B}_{0}$. Thus the gradient coils in the three directions permit spatial information to be obtained. Switching between the three gradients allows for image data signals to be obtained. Simplifying from Equation 1.8 and adding gradient fields $\mathrm{G}(\mathrm{t})$ in $\mathrm{x}$ and $\mathrm{y}$ direction, the received signal from an excited slice can be written as:

$$
s(t)=\iint_{x y} m(x, y) e^{-i 2 \pi k_{x}(t) x} e^{-i 2 \pi k_{y}(t) y} d x d y
$$

Thus the intensity of the signal is proportional to the magnitude of the magnetization.

The above equation can be compared with 2-D Fourier transform of m(x,y) given as:

$$
M\left(k_{x}, k_{y}\right)=\iint_{x} m(x, y) e^{-i 2 \pi\left(k_{x} x+k_{y} y\right)} d x d y
$$

From Equation 1.10 and 1.11 we obtain the signal equation as:

$$
\mathrm{s}(\mathrm{t})=\mathrm{M}\left(\frac{\gamma}{2 \pi} \int_{0}^{\mathrm{t}} \mathrm{G}_{\mathrm{x}}(\tau) \mathrm{d} \tau, \frac{\gamma}{2 \pi} \int_{0}^{\mathrm{t}} \mathrm{G}_{\mathrm{y}}(\tau) \mathrm{d} \tau=\mathrm{M}\left(\mathrm{k}_{\mathrm{x}}, \mathrm{k}_{\mathrm{y}}\right)\right.
$$

Thus at any given time, the signal $\mathrm{s}(\mathrm{t})$, equals the value of the 2D Fourier transform of $\mathrm{m}(\mathrm{x}, \mathrm{y})$. Thus the total signal obtained $\mathrm{s}(\mathrm{t})$, is mapped to a trajectory through Fourier space. In MRI, raw data is stored in 2D Fourier space called " $\mathrm{k}$ " space, where $\mathrm{k}$ represents the spatial frequency variable. In order for the signal to be reconstructed, the trajectories of $\mathrm{s}(\mathrm{t})$ need to cover a sufficient amount of $\mathrm{k}$ space. The $\mathrm{k}$ space data is then 2-D Fourier transformed to obtain the MR image. 


\section{Pulse Sequences}

A pulse sequence is a term used to describe the ensemble of RF pulses (and gradient magnetic field pulses) of defined time and amplitude repeated a specified number of times, resulting in the accumulation of MR signal which is then Fourier transformed to form the MR image ${ }^{6}$. The most common types of pulse sequences used are: spin-echo (SE) and gradient-recalled-echo (GRE). The type of pulse sequence chosen also determines the contrast observed in the MR image.

\section{Spin-Echo Pulse Sequence (SE):}

The spin-echo pulse sequence commences with a 90 degree RF pulse which shifts the magnetization vector into the transverse plane yielding a free induction decay (FID) signal. A slice select gradient is applied during this time in order to selectively excite the protons in the desired slice. Next, a phase encoding gradient is turned on for specified time period and switched off. This gradient is used to distribute the phase of the spins according to their spatial locations. The amplitude of the gradient is varied as seen in Figure 1.5 in order to spatially encode the spins within the slice. At a time of TE/2 after the 90 degree pulse, a 180 degree RF pulse is applied to the slice protons to achieve spin rephasing of the FID signal. "Echoes" (transverse magnetization reappears and forms an echo) are obtained at time TE after the initial 90 degree pulse. It can be seen from Figure 1.5 that the slice select gradient is turned on again during the 180 degree RF pulse. This gradient is particularly useful for multislice spin-echo imaging. Further echoes can be obtained by applying additional 180 degree pulses and this is called fast spin-echo imaging. The final step of spin-echo imaging is the readout of the echo signal. The readout gradient is applied while data acquisition takes place. During the read out gradient, frequency encoding of the spins along the readout direction takes place. The echo-to-echo amplitude decays as a function of $T_{2}$. Spin-echo sequences can be used to obtain $\mathrm{T}_{1}$-weighted images, $\mathrm{T}_{2}$-weighted or proton density weighted images (PD). A spin-echo pulse sequence is shown below: 


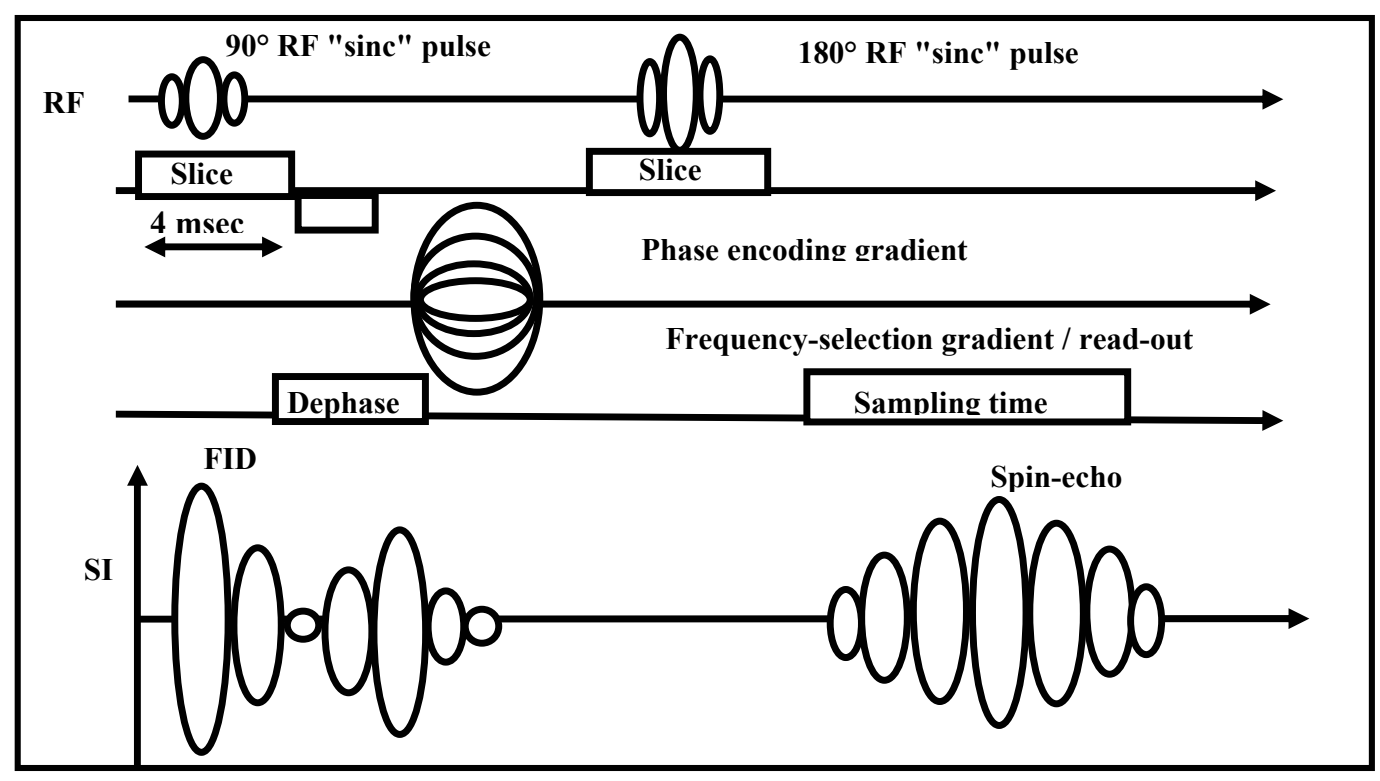

Figure 1.5 Spin-echo pulse sequence ${ }^{1}$.

\section{Gradient-Recalled-Echo Pulse Sequence (GRE):}

Gradient-recalled-echo imaging reverses the polarity of the gradient field rather than using a 180 degree RF pulse to invert the spins to generate an echo as seen in Figure 1.6. A gradient reversal in the readout direction is used. Hence the signal intensity for a given TE is a function of the effective $T_{2}$, called $T_{2}{ }^{*}$, rather than $T_{2}$ as in spin-echo method. The advantage of using a gradient-echo method is that it is a faster imaging method with sensitivity to $\mathrm{T}_{2}{ }^{*}$ contrast and also uses reduced flip angle and gradient reversal instead of a 180 degree RF pulse. It uses a shorter echo time (TE) and without the rephasing RF pulse, dephasing occurs more rapidly in gradient-echo imaging. The gradient-recalledecho method is particularly sensitive to signal loss at boundaries of bone and tissue or air and tissue. In spin-echo sequence, the use of gradient pulses increases the length of the echo time (TE) and uses long TRs which increase the scan time. A gradient-recalled-echo sequence is shown in Figure 1.6. 


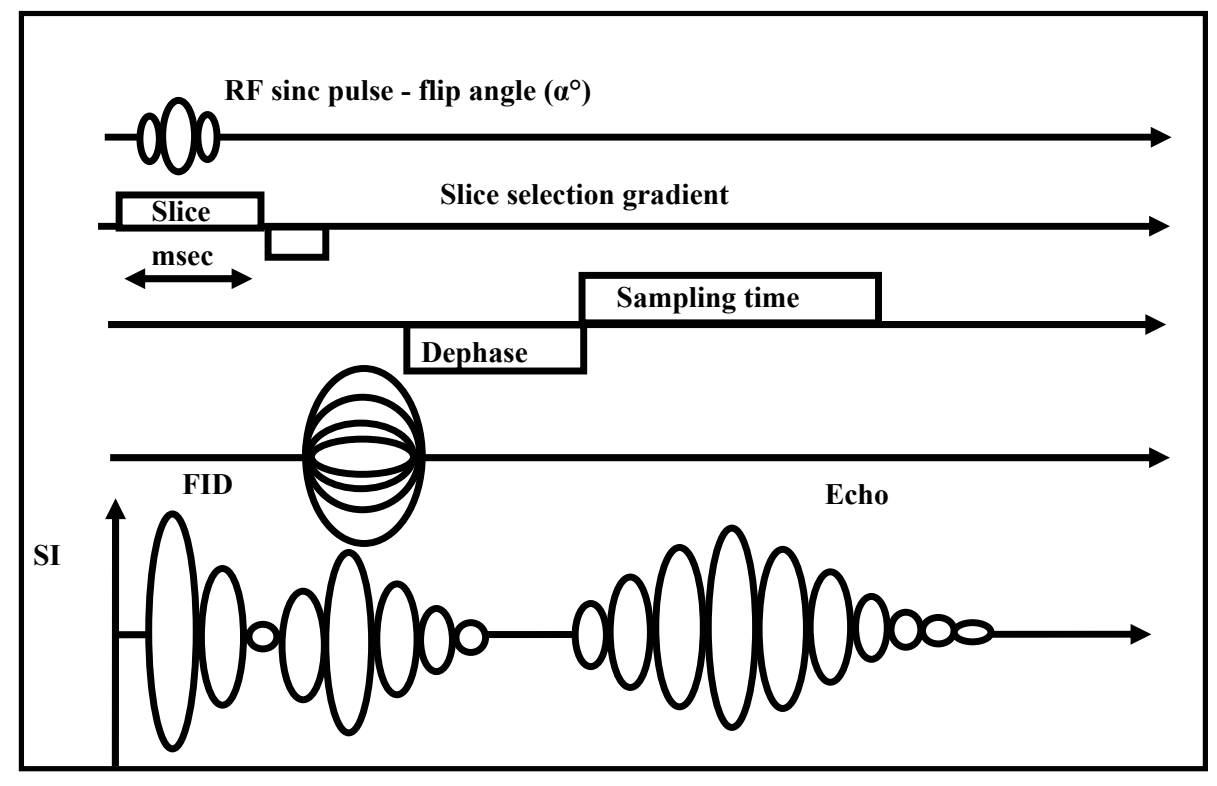

Figure 1.6 Gradient-recalled-echo pulse sequence ${ }^{1}$.

In this thesis the segmentation algorithm is applied to whole brain MR images with tumors. In order to understand the clinically important characteristics to appropriately segment the tumor tissues, it is necessary to understand the tumor types, physiology and metabolism of tumors.

\section{Brain Tumors}

A tumor is a growth of abnormal cells reproducing themselves in an uncontrolled manner. Brain tumors occur either in the brain (as primary brain tumors) or can spread from other parts of the human body to the brain (metastatic brain tumors). There are two types of brain tumor according to the National Cancer Institute ${ }^{7}$ :

- Benign brain tumor consists of abnormal cells and such tumors have distinct boundaries. The cells are slow growing and do not spread or infiltrate and can be removed if they are not located in sensitive areas of the brain.

- Malignant brain tumor consists of cells that have no distinct separation from healthy cells. These cells destroy the surrounding tissues and then spread to other parts of the brain in an uncontrollable manner. 
Most brain tumors are named after the cells from which they arise. Some of the primary brain tumors are: astrocytomas, glioblastoma, oligodendroglioma, and ependymoma. The World Health Organization (WHO) has developed a scheme for classifying and grading tumors. The grading is based on factors such as location, cell types, growth of blood vessels (vascular proliferation), dividing cells (mitotic activity), pathological variation in the appearance of the cells (nuclear atypia) and amount of dead cells or tissues (necrosis) $^{8}$. The different types of brain tumors according to their grade are shown in Table 2.

Table 2: Tumor Grade ${ }^{8}$

\begin{tabular}{|l|l|}
\hline \multicolumn{1}{|c|}{ Grading } & \multicolumn{1}{c|}{ Histological criteria } \\
\hline Grade I \& II & Exhibit nuclear atypia \\
\hline Grade III & High cellular density, nuclear atypia, mitotic activity \\
\hline Grade IV & $\begin{array}{l}\text { Nuclear atypia, mitotic activity, proliferation \&/or } \\
\text { necrosis. }\end{array}$ \\
\hline
\end{tabular}

Low grade tumors (Grade I) of the brain are generally not well encapsulated unlike other tumor types but exhibit slow growth. Since they are slow growing and are relatively noninvasive they are considered to be relatively benign.

Grade II tumors are also considered to be slow growing but they are said to have more poorly defined margins. Surgical treatments for these tumors are difficult as their boundaries are poorly defined.

Grade III tumors have a faster growth pattern and they are called “anaplastic”. These tumors exhibit histological signs of nuclear atypia and mitotic activity. Many low grade tumors develop slowly into anaplastic tumors. These tumors grow their own blood vessels (angiogenesis) and invade the surrounding tissues. 
Grade IV tumors are said to be highly malignant and exhibit very rapid growth. Grade IV tumors also exhibit histological signs such as mitotic activity, proliferation, and/or necrosis. The existing knowledge about each tumor helps the clinicians in determining the course of treatment at each step.

\section{Types of Brain Tumors}

About 50 percent of the primary brain tumors in adults are astrocytomas. These arise from the astrocyte cells that are fibrillary and attach to the blood vessels. Astrocytomas occur primarily in the cerebral hemispheres. Most of the low grade astrocytomas also known as pilocytic astrocytoma are usually non-infiltrating tumors. They are usually considered benign and surgery can remove most of this cyst-like tumor. Grade II astrocytomas are infiltrating although they grow slowly. These astrocytomas are grouped according to their appearance and behavior. Some examples of grade II astrocytoma are: fibrillary, gemistocytic, and protoplasmic. Even after removal of this type of tumors, they might recur as high grade tumor. About 33 percent of the infiltrating astrocytomas mutate and progress from low grade astrocytoma to anaplastic astrocytoma (malignant). The anaplastic astrocytomas have projections like tentacles that grow into the surrounding tissues making it more difficult to completely remove them. About 20 percent of all the primary brain tumors are the grade IV glioblastoma multiforme (GBM). They are also known as grade IV astrocytomas. The glioblastoma tumors are capable of very rapid growth and usually contain necrotic cells and blood vessels.

Gliomas are cancerous cells originating from glial cells. Malignant gliomas show prominent proliferation of microvasculature and have the ability to form blood vessels (angiogenesis). An oligodendroglioma tumor arises from an oligodendrocyte cell and an ependymoma arises from ependymal cells of the brain.

Tumor metabolism is influenced by many factors of which tumor vasculature is one important factor ${ }^{9}$. Once tumor starts to grow rapidly, the tumor cells induce vascular 
growth called tumor angiogenesis, a process of formation of new blood vessels from existing normal blood vessels. The smallest vessels, such as the capillaries, arterioles, and venules known as microvasculature assists the growth of tumor cells by supplying nutrients and oxygen.

Vascularization largely varies the physiology and metabolism of tumors resulting in hypoxia, substrate deprivation, and $\mathrm{pH}$ variations. Tumor vasculature is more chaotic; leaky and not enervated ${ }^{10}$ and hence there is an insufficient supply of oxygen and nutrients to the tumors. The amount of oxygen in the tumor can be of significant importance as the amount of oxygen supply to tumor cells can affect the therapy treatment since hypoxic tumor cells are more resistant to therapy than well-oxygenated tumors. This is due to the fact that cellular damage caused by ionization radiation largely depends on the concentration of oxygen in tissues ${ }^{11}$. Hence it becomes important to estimate the oxygen content in tumor cells. Imaging techniques help to analyze the entire lesion for further treatment. In addition, determining the amount of necrotic (dead) tissue has implications for understanding the efficacy of the treatment and the vascular supply to the tumor.

MR based imaging techniques are used to characterize brain tumors according to their anatomy and physiology, particularly clinicians are interested in determining tumor location, extent, amount of necrosis, vascular supply, and associated edema. There are different imaging techniques that are useful. The specific aim of these techniques is to provide a relevant differential diagnosis. The various techniques used today for imaging brain tumors are listed below and is explained in detail in Chapter 2 .

$>$ Fast fluid-attenuated inversion-recovery (FLAIR) imaging.

$>$ Diffusion-weighted imaging (DWI).

$>$ Perfusion imaging (PWI).

$>$ Gd-DTPA enhanced contrast imaging

MR spectroscopy imaging. 


\section{Chapter 2}

\section{MR Imaging Methods}

MRI, known for its soft tissue contrast, is very useful in evaluating brain tumors. MRI based functional techniques and spectroscopic techniques provide more physiological information than the standard $\mathrm{T}_{1}$-weighted and $\mathrm{T}_{2}$-weighted imaging. Functional imaging provides information about vasculature and tissue perfusion, and optimizes tumor characterization. Following radiotherapy treatments, some tumors may not enhance even while imaging with contrast medium or the enhancement may not indicate malignancy. The concentration curves (rCBV) using perfusion imaging help in quantifying the regional blood flow and volume of a given tumor region. These concentration curves and quantifications will help to determine the remaining tumor region or reoccurrences. MR spectroscopy and spectroscopic imaging allows for measurement of the metabolites of the tumor tissue. These improved techniques give insight to the tumor vasculature, its metabolism, permeability and microcirculation. Different methods of imaging are applied in the clinical environment according to the tumor type and diagnostic requirements. Some of the methods used in this thesis work are described in detail below.

\section{Fast Fluid - Attenuated Inversion-Recovery (FLAIR)}

Fast fluid - attenuated inversion - recovery is a pulse sequence that produces $\mathrm{T}_{1}$-weighted or $\mathrm{T}_{2}$-weighted images with the cerebrospinal fluid (CSF) nulled. This is done by applying an inversion RF pulse before the regular 90 degree pulse sequence. Since the CSF signal is removed, lesions may appear hyperintense in these images. FLAIR is very useful in differentiating and detecting brain tumors. FLAIR is superior to $\mathrm{T}_{2}$-weighted images in distinguishing tumor and edema even though it has the drawback that periventricular tumors may go undetected ${ }^{12}$. 


\section{Diffusion - Weighted Imaging (DWI)}

Diffusion - weighted imaging (DWI) is a MRI technique in which contrast within the image is based on the movement of water molecules. The notation diffusion represents the random movement of molecules also known as Brownian motion. In pure water, the diffusion of water molecules is completely random; however in a cellular environment diffusion is not completely random and is restricted by cellular boundaries and macromolecules.

Using MR imaging, the diffusion properties of water molecules can be measured ${ }^{13}$. In clinical imaging, the tissue diffusion coefficient is denoted by a quantity called the apparent diffusion coefficient (ADC). ADC values are calculated as a representation of relative diffusion restriction. Diffusion is the highest in the ventricles which contain the cerebrospinal fluid (CSF) in the human brain. Hence, in DWI of the normal brain, the ventricles appear less intense than brain parenchyma since diffusion is higher in ventricles compared to parenchyma ${ }^{14}$. Diffusion-weighted MRI sequences are formed by adding a pair of magnetic field gradients to the spin-echo or spin-echo echo-planar pulse sequences. The first gradient is applied after the initial 90 degree pulse as shown in Figure 2.1. This dephases the spins. After the 180 degree pulse, a second gradient is applied which opposes the effect caused by the first gradient. If there is movement of the water protons between the two gradients, refocusing of the moving spins is incomplete, which will result in reduced signal intensity (in areas of high diffusion). The amount of diffusion sensitivity to a particular sequence is denoted by a b-value and is defined in Equation 2.2. A higher b-value provides stronger diffusion weighting. The signal intensity at a particular voxel in a diffusion-weighted MR image can be represented by Equation 2.1 and is called the Stejskal-Tanner equation ${ }^{15}$.

$$
\begin{aligned}
& \mathrm{SI} \approx \exp \left[-\gamma^{2} \delta^{2} \mathrm{G}^{2}\left(\Delta-\frac{\delta}{3}\right) \mathrm{ADC}\right] \\
& \mathrm{b}=(\gamma \mathrm{G} \delta)^{2}(\Delta-\delta / 3)
\end{aligned}
$$


Where,

b - Amplitude and timing parameters of the gradient pulses,

G - Amplitude of the gradient pulses,

$\delta$ - Duration of the gradient pulse,

$\Delta$ - Time interval between gradient pulses,

$\gamma$ - Gyromagnetic ratio.

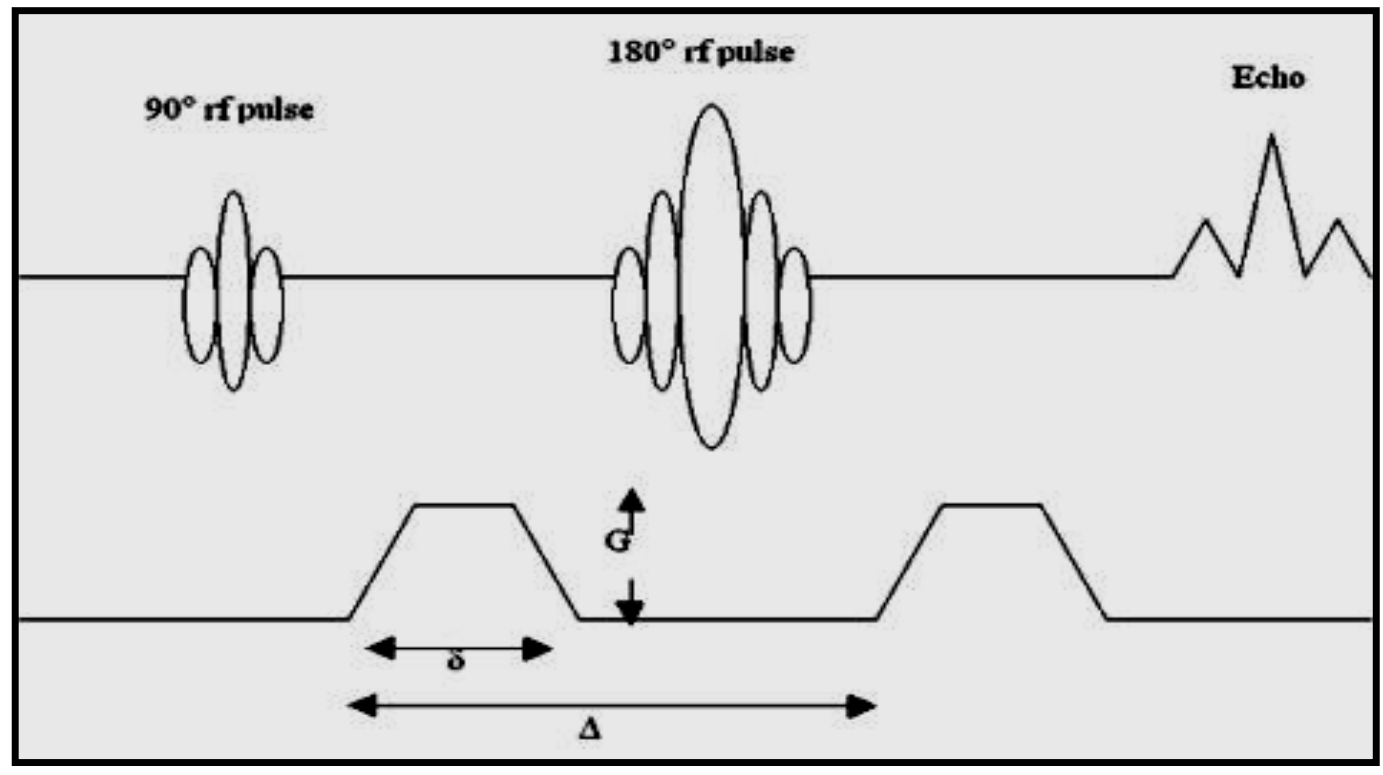

Figure 2.1 Diffusion - weighted imaging pulse sequence ${ }^{15}$.

The attenuation caused by diffusion is calculated by comparing the images between the two b-values (say $b=0$ and higher $b$ value). ADC maps are then calculated by fitting $a$ linear least-squares analysis on a set of diffusion-weighted images.

\section{Diffusion - Weighted Imaging of Tumors}

Diffusion-weighted imaging helps in tumor grading and also in differentiating tumors from edema. Previous studies have correlated the ADC values to tumor cellularity and signal intensity ${ }^{16}$. It has been shown that highly cellular gliomas (higher grade) display lower ADC values than low grade gliomas due to restricted movement of water molecules. Thus the signal intensity in these regions is high. Areas of necrosis display higher ADC values than tumor regions. This is due to the fact that necrosis contains dead 
tissue and hence may offer less impedance to water movement than viable tumor. The ADC values may help in delineating tumor tissue types. However, ADC values alone cannot be used to differentiate tumors from edema but may be used to make a differential diagnosis along with other clinical imaging techniques.

\section{Perfusion - Weighted Imaging (PWI)}

Perfusion imaging measures the rate of delivery of a given quantity of blood to a particular region of interest. Perfusion imaging involves the measurement of microvascular parameters like Cerebral Blood Flow (CBF), Cerebral Blood Volume (CBV) and Mean Transit Time (MTT). This technique is particularly useful in the noninvasive assessment of brain tumors. Imaging is performed using either exogenous or endogenous contrast agents. By measuring the concentration of a tracer agent in the region of interest and measuring the arterial input function, tissue perfusion can be determined. Gadolinium Diethylene-Triamine Pentaacetic Acid (Gd-DTPA) is the most commonly used intravenous exogenous contrast agent in MRI. Gadolinium (Gd) is a metal ion with seven unpaired electrons which produce a large magnetic moment. The unpaired electrons cause faster relaxation of magnetization. Since the $T_{1}$ of the local water protons reduces as gadolinium is injected into the human body, the signal in $\mathrm{T}_{1}$ weighted images is increased ${ }^{17}$. The contrast agent Gd-DTPA cannot cross the blood brain barrier in normal brain and hence there is little relaxation effect and in the first pass it behaves as an intravascular agent. The change in the $T_{1}$ relaxation rate is much larger than that of $\mathrm{T}_{2}$ and hence they are commonly known as $\mathrm{T}_{1}$ agents. When a GRE sequence is used, a $\mathrm{T}_{2}{ }^{*}$ signal loss occurs. This is due to the fact that the high magnetic moment of the gadolinium causes changes in the susceptibility of the blood as compared to the surrounding tissue ${ }^{18}$. The signal loss due to gadolinium is because the inhomogeneous field created additional dephasing of the spins, hence reducing the $\mathrm{T}_{2}{ }^{*}$ signal. Spin echo imaging can also be used, but it is more sensitive to the changes in the capillaries than the larger blood vessels. The signal changes can be used to quantify the local concentration of gadolinium by tracer kinetics principles. The application of tracer kinetics principles involves obtaining the concentration curve as the first passage of the bolus (Gd-DTPA) 
into the tissue takes place. Quantification can be achieved if the arterial input function is measured or modeled ${ }^{19}$.

\section{Imaging Using Perfusion Techniques}

In our institution, MR contrast agent (Gd-DTPA) is administered at a rate of $5 \mathrm{cc} / \mathrm{sec}$ for 20 seconds through a power injector. As soon as the contrast Gd-DTPA is administered, the tissue enhancement can be seen within 1 minute. The images obtained are then used to generate the relaxivity (the inverse of the relaxation time) versus time curves $\left(\Delta \mathrm{R}_{2}^{*}=\Delta 1 / \mathrm{T}_{2}^{*}\right)$.

$$
\Delta \mathrm{R}_{2}^{*}=-\ln \left[\mathrm{S}(\mathrm{t}) / \mathrm{S}_{0}\right] / \text { echo time }
$$

Where $\mathrm{S}(\mathrm{t}), \mathrm{S}_{0}$ : signal intensities at time $\mathrm{t}$ and baseline.

\section{Cerebral Blood Volume}

Cerebral blood volume is defined as the total volume of blood in given region of interest and is measured in units of milliliters of blood per 100 grams of tissue $(\mathrm{ml} / 100 \mathrm{~g})^{20}$. The relative cerebral blood volume ( $\mathrm{rCBV}$ ) maps are obtained by fitting a gamma variate function to the relaxivity $\Delta \mathrm{R}_{2}^{*}$ transit curve for each pixel. The rCBV is calculated as:

$$
\mathrm{rCBV}=\int_{0}^{\infty} \Delta \mathrm{R}_{2}(\mathrm{t}) \mathrm{dt}
$$

It is independent of the shape of the time course response. The rCBV has arbitrary units and is generally normalized to another tissue hence expressed as \% of the control region. Figure 2.2 shows schematically the procedure to obtain an rCBV map. 


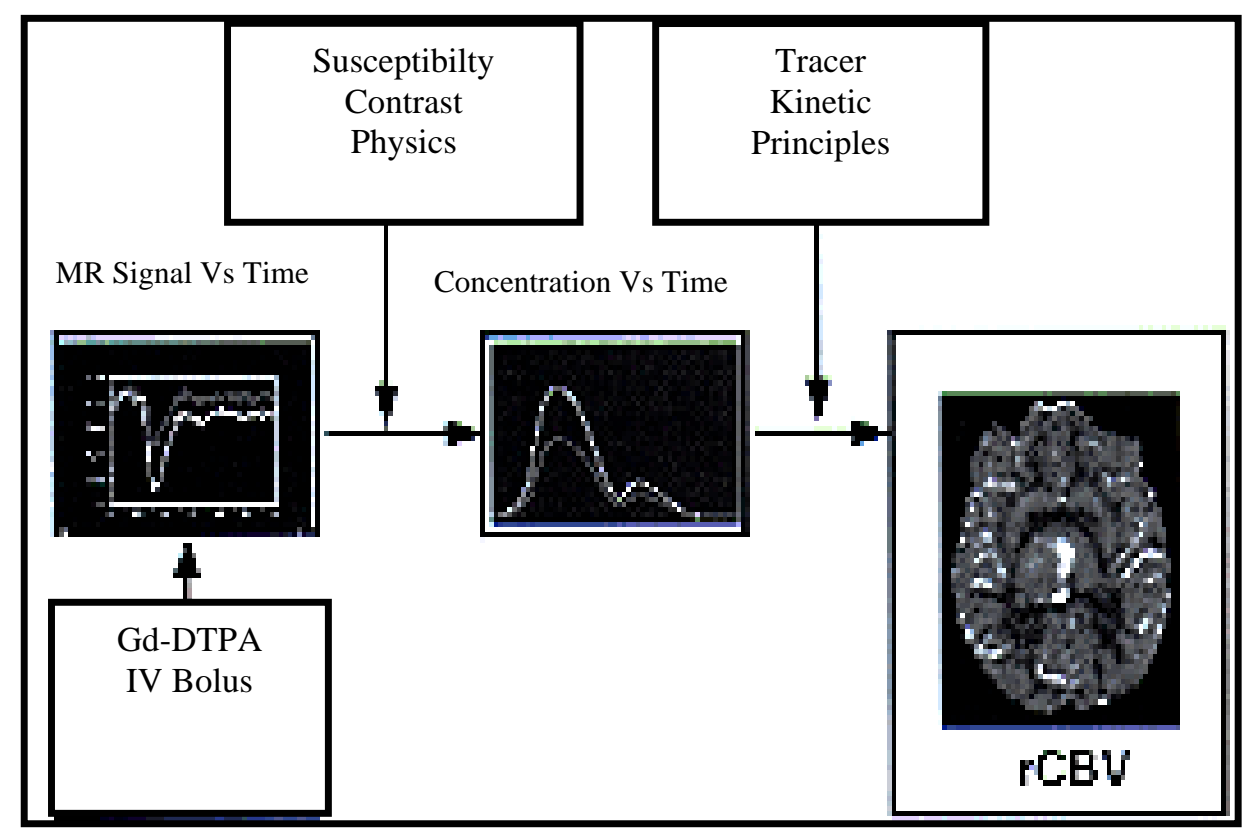

Figure 2.2 Methodology for perfusion imaging ${ }^{21}$.

\section{Mean Transit Time}

Mean transit time is defined as the average of the transit time of blood through a given brain region, integrated across the different paths. The transit time through brain tissues is usually about 4 seconds. Practically it is difficult to measure MTT because the bolus injection may not be instantaneous. The relative mean transit time (rMTT) is obtained as the normalized first moment of the gamma variate fitted curve, up to the peak of the curve expressed in units of seconds.

$$
\text { rMTT }=\frac{\int_{0}^{\text {tpeak }} \mathrm{t}^{*} \Delta \mathrm{R}_{2}(\mathrm{t}) \mathrm{dt}}{\int_{0}^{\text {tpeak }} \Delta \mathrm{R}_{2}(\mathrm{t}) \mathrm{dt}}
$$

\section{Cerebral Blood Flow}

Cerebral blood flow is defined as the volume of blood flowing through a given brain region per unit time. The CBF is measured in units of milliliters of blood per $100 \mathrm{~g}$ of brain tissue per minute (mL/100 g/min). Normally CBF is $60 \mathrm{ml} / 100 \mathrm{~g}$ brain tissue/min. As the Gd-DTPA contrast passes through larger blood vessels and diffuses into the 
surrounding tissues, the changes obtained can be used to calculate the relative CBF (rCBV) maps using the central volume theorem as:

$$
\mathrm{rCBF}=\mathrm{rCBV} / \mathrm{rMTT}
$$

rCBV is expressed in arbitrary units.

\section{Perfusion Imaging Of Brain Tumors}

Perfusion imaging can be an indicator of tumor angiogenesis which is associated with tumor growth. Perfusion imaging has been very useful in stereotactic biopsy guidance, differentiating necrosis from tumor. Relative CBV values only reflect the degree of vascularity and do not provide a direct measurement of blood volume. It has been shown that with histological confirmation, higher rCBV values indicate higher grade tumor and higher mitotic activity ${ }^{22}$. Low grade tumors which have low CBV demonstrated less contrast enhancement in tumor regions when compared to high grade tumors ${ }^{22}$.

\section{MR Spectroscopy Imaging}

\section{Chemical Shift}

The difference between the resonant frequency of a nucleus and a standard, relative to the standard is known as chemical shift. Both water and fat in the human body contain hydrogen. The chemical shift difference between these two types of hydrogen containing substances is approximately $3.5 \mathrm{ppm}$. The concentration of most metabolites present in the human body is typically orders of magnitude less than that of the water or fat signal in tissues.

\section{Spectroscopy Imaging}

Spectroscopic imaging provides information about the tissue metabolite concentrations in a region of interest. Also known as chemical shift imaging (CSI), this method produces an image from one chemical shift component in a region of interest after suppression of water and/or fat. Spectroscopic information over multiple regions is processed to create maps of the metabolite concentration. In order to obtain the spectroscopic information, suppression techniques are used to eliminate the larger water signal and a spectral 
dimension is added to the usual MR procedure. The gradients through their range of values are used to record the spectra from all points in the spatial-spatial domain. The spectra obtained are presented as a series of peaks based on the chemical shift relative to a standard (tetramethyl silanie). The peaks obtained in normal brain ${ }^{23}$ are: $\mathrm{N}$ - acetyl aspartate (NAA) at $2.0 \mathrm{ppm}$, choline at 3.2ppm, creatine at $3.0 \mathrm{ppm}$, and other minor peaks are obtained between 2.0-2.2 ppm. The NAA is an indicator of neuronal activity in the brain. Creatine is thought to be an indicator of energy, choline, a neurotransmitter, appears to be an indicator of membrane turnover ${ }^{23}$. Since the $T_{1}$ and $T_{2}$ are related to each peak, the height of the peaks obtained depend on the parameters chosen for TE and TR. Additional peaks are thus obtained at short echo times as seen in Figure 2.3. The concentration of the metabolites is proportional to the area under the peak for each metabolite after correction for $\mathrm{T}_{1}$ and $\mathrm{T}_{2}$ decay.

\section{Spectroscopy Imaging Of Brain Tumors}

Many studies have indicated the potential of spectroscopic imaging in diagnosing brain tumors $^{24}$. Comparison of relative peak heights may be used to indicate abnormalities in the brain. Studies have shown that brain tumors have elevated choline, decreased NAA, and decreased creatine peaks ${ }^{25}$. Decreased NAA levels in brain tumors indicate the lack of neurons (neuronal death) in glial neoplasm. Choline signal was found to be highly elevated in grade II and grade III tumors. High grade glioblastomas demonstrate a low creatine peak and in addition to these peaks, a lipid peak at 0.9-1.2ppm and a lactate peak at $1.3 \mathrm{ppm}$. One such set of spectra comparing normal tissue to tumor tissue is shown in Figure 2.3 and Figure 2.4. In Figure 2.3, left image shows the $T_{2}$-weighted image of a tumor patient and right image is the spectrum under the square voxel. The square voxel is chosen in a normal tissue region so the spectrum of metabolites obtained should have increased NAA, decreased choline, and increased creatine peak as seen below. When the voxel is chosen in the tumor tissue region in Figure 2.4, the spectrum of the metabolites is seen as described for tumors above. 


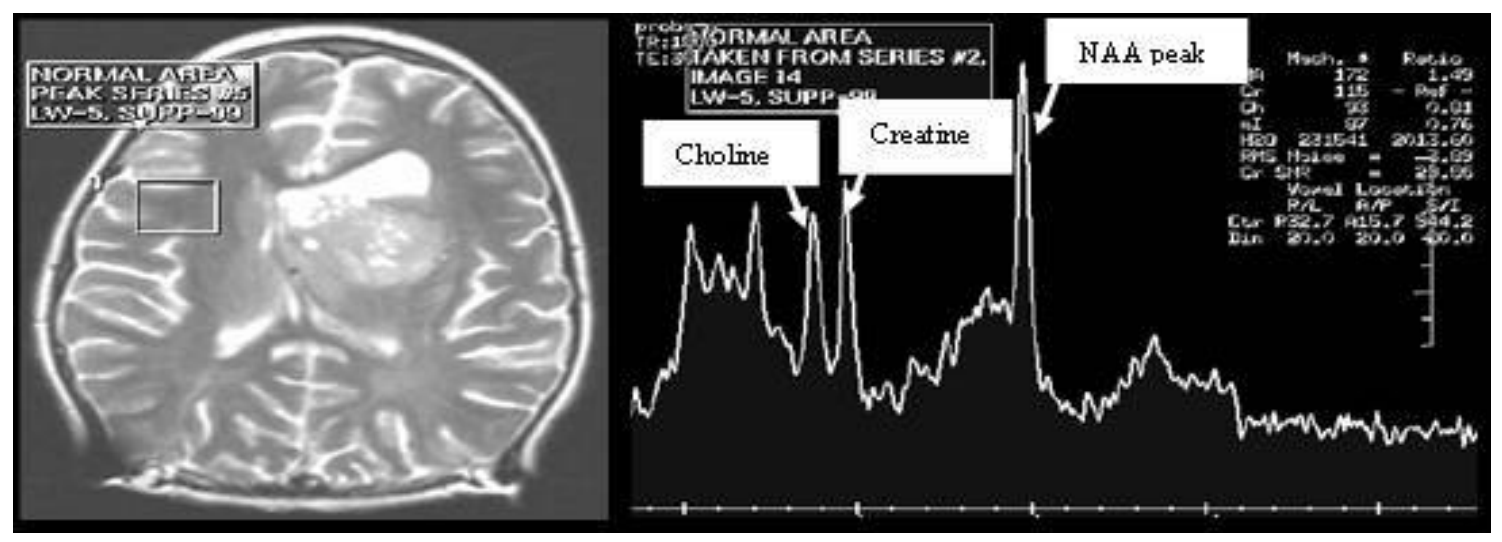

Figure 2.3 Spectroscopic image of tumor patient with voxel at normal tissue and its spectrum.

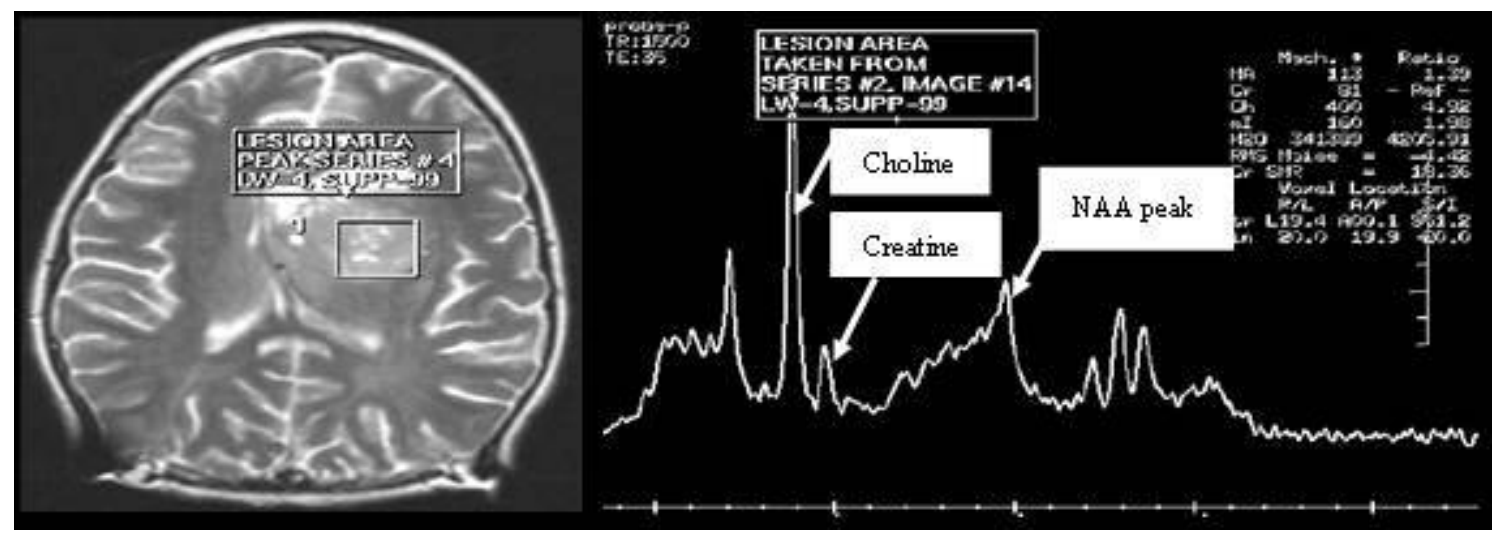

Figure 2.4 Spectroscopic image of tumor patient with voxel at tumor tissue and its spectrum.

\section{Segmentation Using Fuzzy C - Means Clustering Algorithm}

Segmentation is the process of dividing an image into meaningful regions. MR image segmentation of brain or regions of interest can be used to extract different tissues. Segmentation in MR images may help in providing better visualization and good separation between tissues especially the tumor regions. In segmentation techniques, both supervised and unsupervised classification algorithms have been applied to MRI ${ }^{26}$.

Supervised and seeding techniques require human intervention. An operator familiar with human anatomy is required to outline the regions containing gray matter, white matter, etc., and assign a physical label (color) to that region. Then the algorithm is trained and data is segmented. Since the anatomical structures differ for each subject this process is 
usually time consuming and difficult. The results produced by this method may vary with operators and hence may not be reliable and reproducible. Only trained operators may be able to use this method which is one of the disadvantages. But since the algorithm is trained and then data is segmented they may yield better results where operator intervention errors and time can be compromised.

In the unsupervised approaches, the operator is required to assign tissue class labels to the colored segmented image data sets and not the original MRI data sets. Unsupervised segmentation methods are usually fast and do not require operator intervention to segment images hence reducing inter-user variability. Unsupervised algorithms are usually based on clustering algorithms and can be easily extended to multidimensional data sets. The fuzzy c-means (FCM) algorithm is a type of clustering algorithm that can perform segmentation on MR images based on pixel classification method. This may yield better results and provide better distinction between anatomical tissues than other unsupervised methods.

In this thesis, a software tool is developed for applying unsupervised FCM clustering algorithm to a combination of whole volume MRI data sets taken on normal subjects and patients with tumors. The results are then compared with the available software SPM2 ${ }^{27}$. Most of the previous work using FCM segmentation on MRI images ${ }^{28-30}$ has not included any "physiological” data sets (DWI, perfusion, and gadolinium-enhanced images).

Clustering is an unsupervised image segmentation technique that divides the given data set into clusters (classes). This division puts similar data objects in the same cluster whereas dissimilar data objects belong to other clusters. That is, " $\mathrm{N}$ " data samples are classified into " $\mathrm{n}$ " clusters. An important component of a clustering algorithm is the distance measure between data points. A Euclidean distance metric is used to group similar or dissimilar data points. Cluster analysis contains a group of different algorithms for classifying the data. These algorithms are based on the similarity measure. 
Some of the clustering techniques are:

- Exclusive clustering - Each data point assigned to only one cluster.

- Overlapping clustering - Each data point can be assigned to one or more clusters. Fuzzy c- means algorithm is a type of overlapping clustering method.

- Hierarchical clustering - Each data point is partitioned in several steps into finer classes using a set of fuzzy rules.

- Probabilistic clustering - For each data point, a probability distribution over the clusters is determined from which the probability of belonging to a cluster is determined.

- Objective function based clustering - Most of the clustering algorithms except hierarchical, involves minimizing the performance index " $\mathrm{J}$ ". Using the sum of the squared distances, the optimum value is obtained through iteration. The sum of the squared distances signifies the performance index.

The fuzzy c-means (FCM) algorithm was established by J. Bezdek and Dunn in $1981^{31}$. This algorithm is widely used in variety of pattern recognition and medical image segmentation applications today ${ }^{32,33}$. FCM is a fuzzy serial, soft segmentation unsupervised pixel classification clustering algorithm. In this algorithm, each data point may belong to more than one class through partial memberships. A membership function specifies the degree to which a data point (pixel intensity) belongs to a cluster and the membership value is the value or the degree of similarity to a cluster. The application of fuzzy sets in classifying MRI data sets causes the class membership to become relative, since an object can belong to several classes at the same time but with different degrees. Previous studies have shown that as the dimension of feature space (multispectral) increases, MRI image segmentation improves ${ }^{34,35}$.

The FCM algorithm uses an iterative approach which minimizes the objective function, $\mathrm{J}$ the sum of weighted distances (Euclidean distance) between each data point, and the cluster center, and searches for compact clusters. All clusters calculated are approximately the same size and each of them is represented by their centers. 


$$
\mathrm{J}=\sum_{\mathrm{i}=1}^{\mathrm{N}} \sum_{\mathrm{j}=1}^{\mathrm{C}} \mathrm{u}^{\mathrm{m}}{ }_{\mathrm{ij}}\left\|\mathbf{x}_{\mathrm{i}}-\mathbf{c}_{\mathrm{j}}\right\|^{2} \quad 1 \leq \mathrm{m} \leq \infty
$$

Where,

$\mathrm{J}$ - Objective function,

$\mathrm{u}_{\mathrm{ij}}$ - Membership matrix - contains all the membership values for each data point $\mathrm{x}_{\mathrm{i}}$ in class j,

$\mathbf{c}_{j}-\mathbf{c}=\left(c_{1}, c_{2}, \ldots, c_{j}\right)-$ Vector of cluster centers where $c_{j}-$ cluster center of class $j$,

C - the number of classes for $1 \leq \mathrm{j} \leq \mathrm{C}$,

$\mathbf{x}_{\mathrm{i}}-\mathrm{i}^{\text {th }}$ of d-dimensional data,

m - Weighting exponent of each membership,

$\|*\|$ is any norm between any measured data and the center.

In every iteration step of the objective function $\mathrm{J}$, the membership $\mathrm{u}_{\mathrm{ij}}$ and cluster centers $\mathrm{c}_{\mathrm{j}}$ are updated by:

$$
\begin{aligned}
& \mathbf{u}_{\mathrm{ij}}=\left[\sum_{\mathrm{k}=1}^{\mathrm{C}}\left(\frac{\left\|\mathbf{x}_{\mathrm{i}}-\mathbf{c}_{\mathrm{j}}\right\|^{2}}{\left\|\mathbf{x}_{\mathrm{i}}-\mathbf{c}_{\mathrm{k}}\right\|^{2}}\right]^{2 /(\mathrm{m}-1)}\right]^{-1} \text {, for all i, } \mathrm{k} \\
& \mathbf{c}_{\mathrm{j}}=\frac{\sum_{\mathrm{i}=1}^{\mathrm{N}} \mathrm{u}_{\mathrm{ij}}^{\mathrm{m}} \cdot \mathbf{x}_{\mathrm{i}}}{\sum_{\mathrm{i}=1}^{\mathrm{N}} \mathrm{u}_{\mathrm{ij}}^{\mathrm{m}}}
\end{aligned}
$$

The iteration stops if $\mathrm{J}(\mathrm{i})-\mathrm{J}(\mathrm{i}-1)<\varepsilon$, where $\varepsilon>0$.

The membership matrix $\mathrm{u}_{\mathrm{ij}}$ contains the membership degree values between $[0,1]$ where 1 represents that the data point $\mathrm{x}_{\mathrm{i}}$ belongs to a cluster $\mathrm{j}$ and a value of 0 represents that the data point does not belong to the cluster $\mathrm{j}$. 
An optimal number of classes must be chosen in order to obtain good segmentation results. In this application, the number of classes represents the number of brain tissue types. The FCM algorithm clusters the data set into the number of classes specified. If more classes are specified it may result in over-segmentation in the case of normal tissues. But in case of abnormal tissues like tumor, more classes may be required to be specified. Hence it is important to know the number of tissue types to be classified. To understand our data set better, the FCM algorithm has been used with different number of classes and compared to find the best possible class number. A cluster center plot of the data set may be helpful in deciding the number of classes. Such a cluster center plot is shown in Figure 2.5.

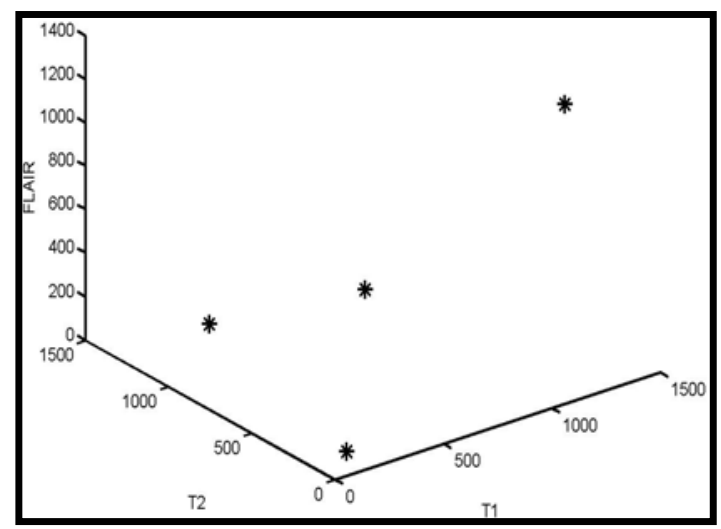

Figure 2.5 3D Cluster center plot with four classes of a simulation dataset.

Another important parameter is the exponent ' $m$ ' which is the degree of fuzziness. For each iteration of the calculation of the objective function, the degree of fuzziness ' $m$ ' needs to be specified. The parameter $\mathrm{m}$ controls the slope of $\mathrm{U}$ thus determining the quantity of class overlapping. The degree of fuzziness $m$ can be any value greater than 1 for the FCM algorithm. As m increases, the membership function $\mathrm{U}$ decreases as seen from Equation 2.5. It has been shown that the greater the $\mathrm{m}$, the fuzzier (greater the overlap) the results ${ }^{36}$. By decreasing the value of $m$ (closer to 1 ) we can obtain crisp membership indicator functions. The different types of membership indicator functions for different $m$ values are shown in Figure 2.6. 


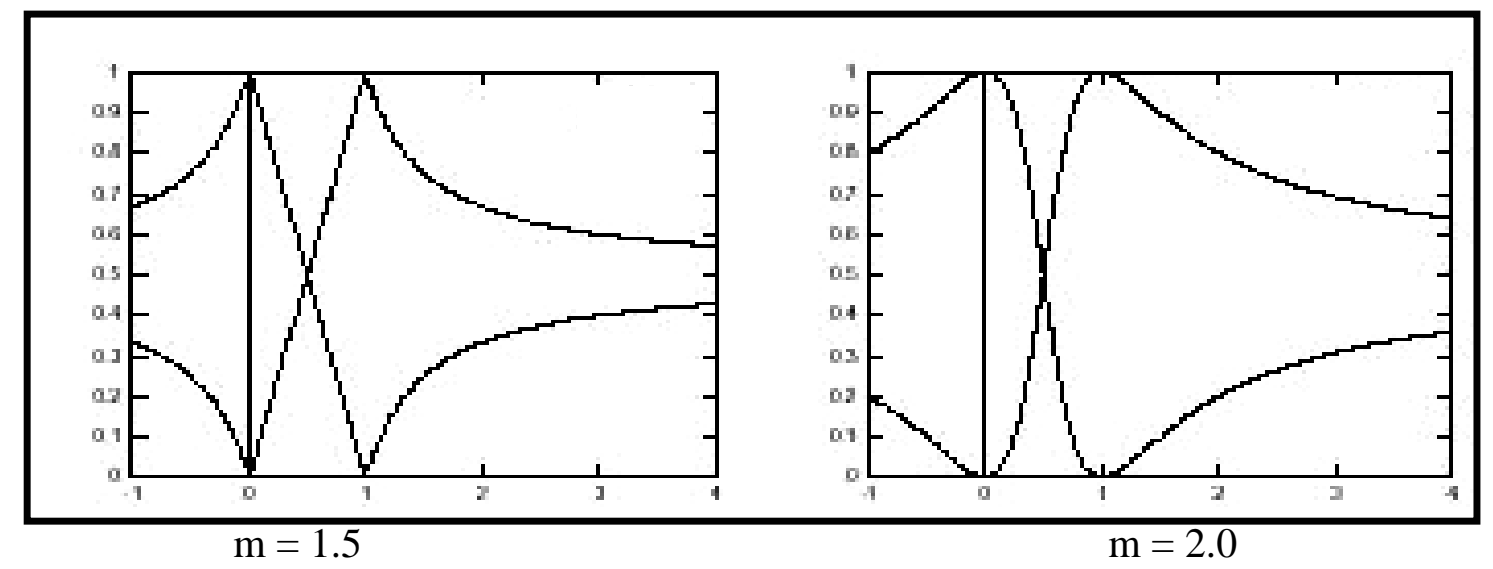

Figure 2.6 Membership functions with respect to $\mathrm{m}^{37}$.

Keeping in mind the above parameters, the ideal number of classes and degree of fuzziness $\mathrm{m}$ for both normal and abnormal subjects were chosen in our algorithm. The iterative process $\mathrm{J}$ is minimized when high membership values (uij) are assigned to pixels whose intensities are closer to the particular cluster centroid and low membership values are assigned to pixels whose intensities are far from the cluster centroid.

The segmented data are then labeled by class (tissue type). At this stage, the pixels are color-coded to differentiate between tissue types. The process called defuzzification uses the maximum membership rule. The maximum value of uij is chosen and if it is greater than a threshold value, the pixels containing those membership values are coded a color (segmented into a class). The plot in Figure 2.7 represents a membership matrix u with threshold of 0.55 for a one-dimensional data set.

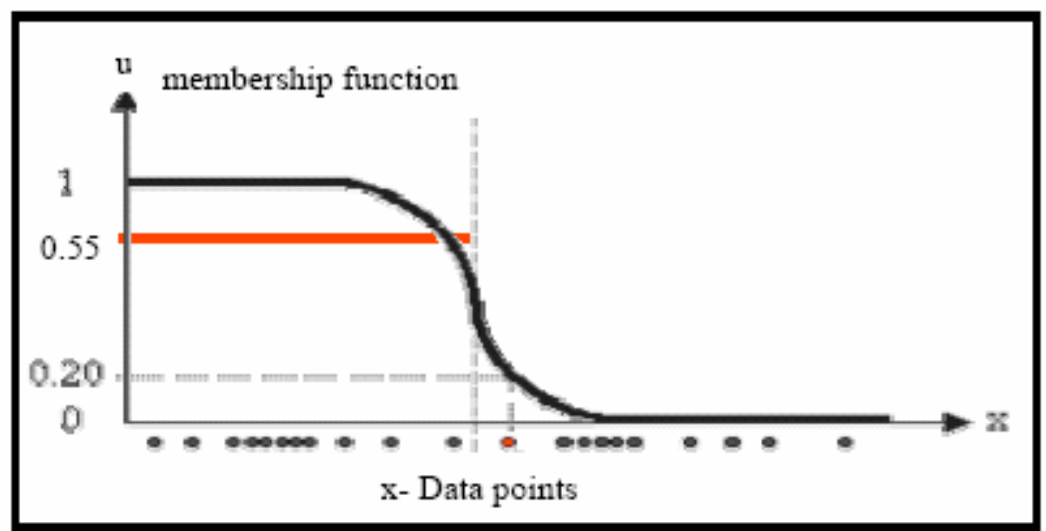

Figure 2.7 Membership function indicating threshold value of 0.55. 


\section{Chapter 3}

\section{Methods}

\section{Simulation Models}

Before pre-processing and segmenting the real MRI data sets, in order to understand our data three types of simulation models were created. They are:

- $\quad$ Simulation using stripes - 7 tissue types

- Partial volume simulation - 3 tissue types

- Brain Region-of-Interest (Brain ROI) - 3 tissue types.

\section{Simulation Using Stripes}

The intensity values of a normal brain of seven different tissue types were measured for $\mathrm{T}_{1}, \mathrm{~T}_{2}$ and FLAIR images. Using these values an 8 stripe matrix was created. This image contained eight tissue types and eight pixels per tissue stripe. Figure 3.1 shows a simulations stripe containing such $\mathrm{T}_{1}, \mathrm{~T}_{2}$, FLAIR image intensities.

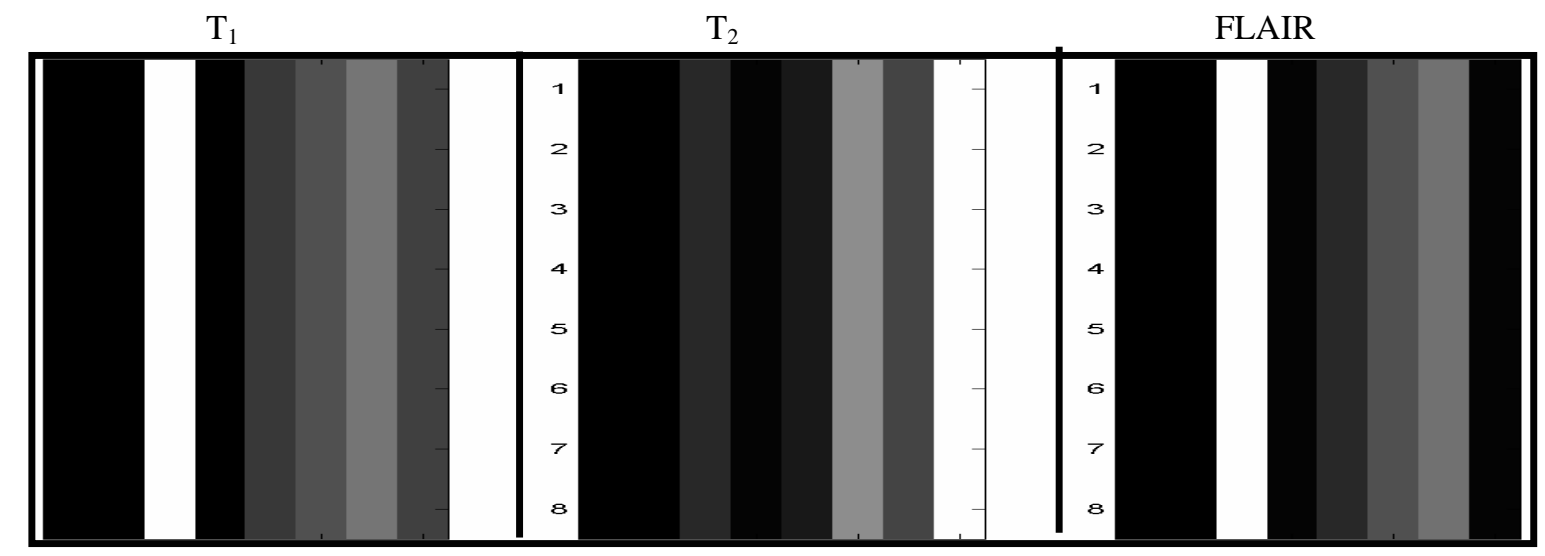

Air, Air, Scalp, Skull, Meninges, Gray Matter, White Matter, CSF

Figure 3.1 Simulation of intensity using stripes

Table 3 shows the values for each tissue type. 
Table 3: Pixel intensity values for simulation

\begin{tabular}{|c|c|c|c|}
\hline Tissue & $\mathbf{T}_{\mathbf{1}}$ & $\mathbf{T}_{\mathbf{2}}$ & FLAIR \\
\hline Air & 40 & 10 & 10 \\
\hline Scalp & 740 & 550 & 330 \\
\hline Skull & 80 & 40 & 20 \\
\hline Meninges & 380 & 220 & 130 \\
\hline Grey Matter & 370 & 480 & 140 \\
\hline White Matter & 570 & 400 & 90 \\
\hline CSF & 350 & 1530 & 50 \\
\hline
\end{tabular}

Using FCM, MATLAB ${ }^{38}$ scripts were written to segment these stripes. A standard color map was developed and each tissue type was assigned a color. The color map can accommodate up to 12 classes (tissues) presently and is shown in Figure 3.2. In this work only six classes (colors) for normal and nine classes (colors) for patients with tumors were used.
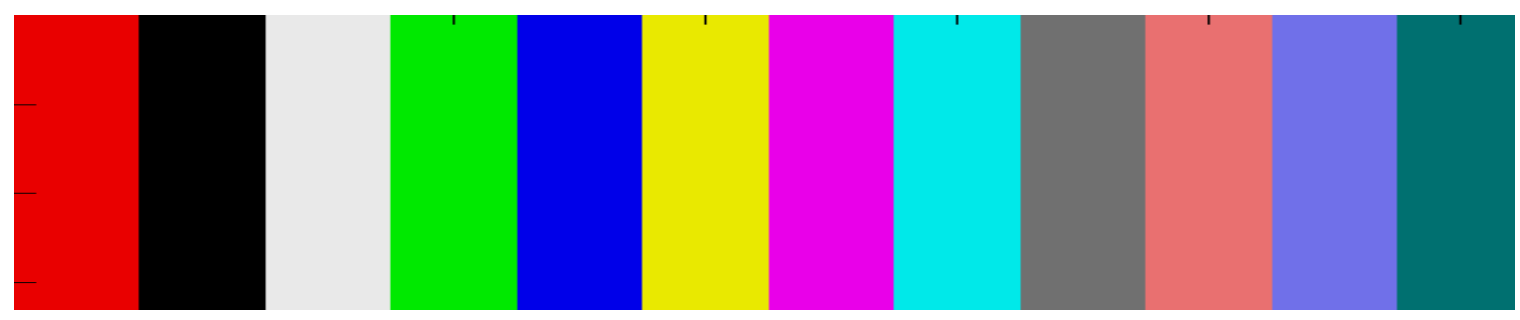

Unclassified Class 1 Class 2 Class 3 Class 4 Class 5 Class 6 Class 7 Class 8 Class 9 Class 10 Class 11

Figure 3.2 Color map

In MR images, thermal noise is the primary source of random noise and is characterized by a Rician noise but can be approximated by Gaussian distribution. Hence we add randomly generated white Gaussian noise to each intensity value in order to simulate noise in the MRI data. The stripes are then segmented using the FCM algorithm in MATLAB. Since each stripe is a tissue (class) perfect segmentation should result in stripes only. The simulation stripes were segmented with and without intensity normalization. In intensity normalization, the intensity values were scaled to the maximum value. The centers calculated from FCM and their membership values were 
plotted and examined for this optimization. The norms of the centers were also calculated and sorted to identify tissue types. The number of inconsistent classifications (incorrect) were calculated as percentage error for each run of FCM and plotted versus threshold for noise levels from 10 increasing to 40 . The simulation stripes are segmented with different number of classes, noise levels, exponent $m$ and thresholds for selecting of the optimal parameters for these types of images.

\section{Partial Volume Simulation}

A partial volume effect in a MR image means voxels may contain a mixture of tissue types. The partial volume effects can be decreased by decreasing the voxel size in a MR image. Shown below in Figure 3.3 a) is an image with $1 \times 1 \times 1 \mathrm{~mm}^{3}$ slice. This image when the resolution is increased to $2 \times 2 \times 2 \mathrm{~mm}^{3}$ as in Figure $3.3 \mathrm{~b}$ ) displays partial volume effects.

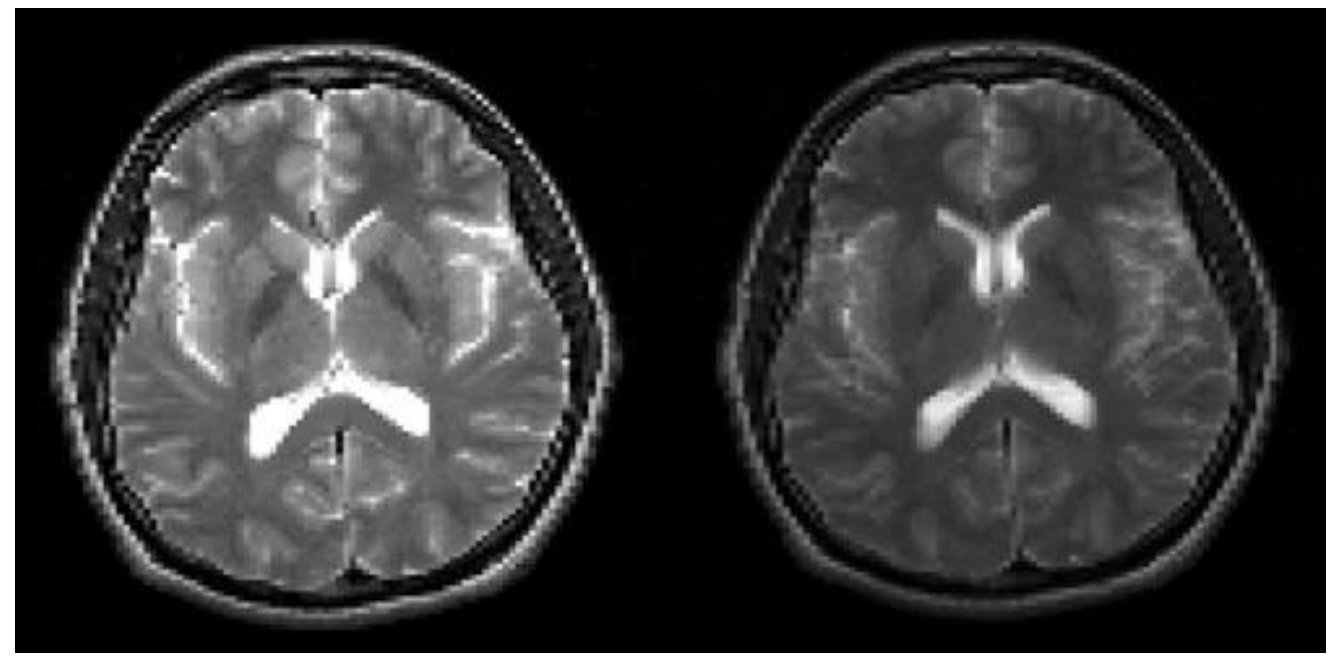

Figure 3.3 Partial volume effects - a) $1 \times 1 \times 1 \mathrm{~mm}^{3}$ image b) $2 \times 2 \times 2 \mathrm{~mm}^{3}$ image showing partial volume blurring.

In order to see how partial volume affects FCM segmentation, partial volume stripes were created for only three types of tissue (gray matter, white matter, CSF). The stripe model is shown below. 


\begin{tabular}{|c|c|c|c|c|c|c|c|c|c|c|c|c|c|c|}
\hline \multirow{2}{*}{ GM } & \multirow{2}{*}{ GM } & \multirow{2}{*}{ GM } & \multicolumn{2}{|c|}{ Avg. } & \multirow{2}{*}{ WM } & \multirow{2}{*}{ WM } & Avg. & \multirow{2}{*}{$\mathrm{CSF}$} & \multirow{2}{*}{$\mathrm{CSF}$} & \multicolumn{2}{|c|}{ Avg. } & \multirow{2}{*}{ GM } & \multirow{2}{*}{ GM } & \multirow{2}{*}{ GM } \\
\hline & & & GM & WM & & & WM $\overline{C S F}$ & & & CSF & GM & & & \\
\hline
\end{tabular}

The simulation model was run for different values of exponent $\mathrm{m}$, noise levels and thresholds. The number of classes was specified as only three since it contained only three tissue types.

\section{Brain Region-of-Interest Simulation}

Next, to check in a real data set how FCM segmentation works, a region of interest (ROI) was selected from the obtained MR images of normal subjects. The ROI was chosen in such a way that it contained only the GM, WM, CSF tissues from the middle of the MR brain image. Figure 3.4 shows a brain ROI of a normal subject.
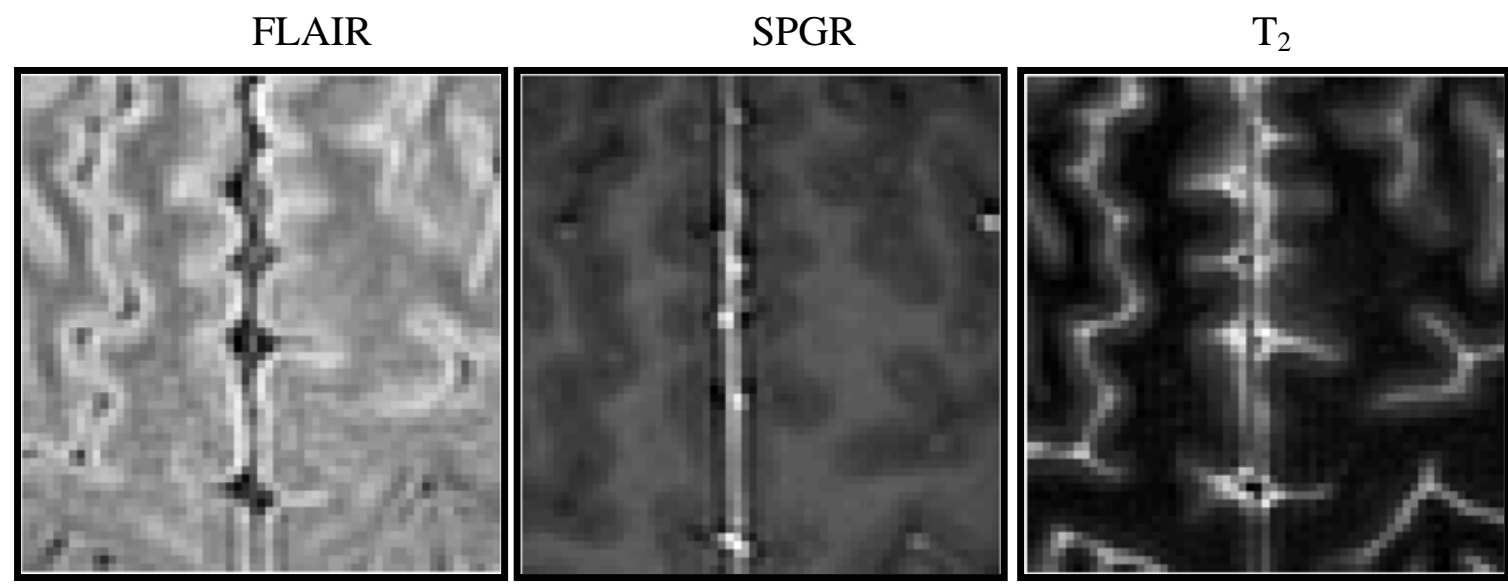

Figure 3.4 Brain ROI

The FCM was then run for this model to see the segmentation results in actual data. Here also the FCM was executed for different values of exponent $\mathrm{m}$ and thresholds. The number of class was fixed at three since there are only three tissue types. The optimized values of $\mathrm{m}$ and threshold from all these simulations were used to segment the actual data sets.

\section{MRI Volume Data Sets}

In this thesis, the FCM clustering approach was applied to multispectral MRI $\left(\mathrm{T}_{2}\right.$, FLAIR, SPGR-pre contrast, SPGR-post contrast, SPGR-post contrast (SPGRGd), DWI) 
image data sets which segmented them into different tissue types including abnormal tissues. So for each pixel $i$, the feature vector was comprised of $x_{i}=\left[T_{2 i}, F_{i}, D_{W} I_{i}, S_{p g r}\right.$, SpgrGd $\mathrm{d}_{\mathrm{i}}$. By adding more image types (multi spectral) the segmentation may improve and provide more information ${ }^{39}$. The feature vector $x_{i}$ is a five-dimensional vector. The feature vectors were then segmented into six classes for normal tissues and nine classes for abnormal tissues. The $\mathrm{m}$ value used on these data sets was set to $\mathrm{m}=1.5$. The optimized threshold value from the simulation data sets $=0.55$ was used for classifying into clusters (tissues). These values were chosen as the best values to include pixels while not mixing classes after inspecting the cluster vector plots and membership function plots. The tissue classes which look like white matter, gray matter and CSF were compared to white matter, gray matter and CSF segmented images from Statistical Parametric Mapping software $^{27}$ (SPM2).

\section{Data Acquisition and Analysis}

Using all the imaging procedures mentioned in Chapter 2, MRI scans were obtained in four normal subjects and five tumor patients. Table 4 below shows the type of tumor for each tumor patient. All tumor patients have undergone radiation therapy, chemotherapy and / or surgery.

Table 4: Tumor types in patient sample.

\begin{tabular}{|l|l|}
\hline Patient & \multicolumn{1}{|c|}{ Tumor type } \\
\hline Patient 1 & Grade IV Glioblastoma Multiforme \\
\hline Patient 2 & Grade II Gemistocytic Astrocytoma \\
\hline Patient 3 & Astrocytoma \\
\hline Patient 4 & Grade II Gemistocytic Astrocytoma \\
\hline Patient 5 & Low grade Astrocytoma \\
\hline
\end{tabular}

The types of MRI scan taken for each subject, and the parameters involved are summarized in Table 5. The total scan time was around 1 hour and 15 minutes.

- Anatomical scans were taken first (3-Plane Localizer, DWI, FLAIR, and $\mathrm{T}_{2}$ ).

- Next, pre-contrast high resolution images (3D SPGR) are obtained. 
- Intravenous contrast Gd-DTPA (20ml) is injected through a power injector. During the first passage of the bolus, perfusion scans are obtained.

- A high resolution (3D SPGRGD) post contrast scan is then obtained.

- Last, a $\mathrm{T}_{1}(\mathrm{GD})$ contrast enhanced image is obtained.

Table 5: Imaging Parameters

\begin{tabular}{|c|c|c|c|c|c|c|c|}
\hline $\begin{array}{l}\text { Series } \\
\text { Name } \\
\text { (view) }\end{array}$ & $\begin{array}{c}\text { Field-of- } \\
\text { view(FOV) } \\
(\mathrm{mm})\end{array}$ & $\begin{array}{c}\# \\
\text { Slices }\end{array}$ & $\begin{array}{c}\text { Slice } \\
\text { thickness } \\
(\mathrm{mm})\end{array}$ & $\begin{array}{l}\text { TR } \\
(\mathrm{ms})\end{array}$ & $\begin{array}{l}\text { TE } \\
(\mathrm{ms})\end{array}$ & $\begin{array}{c}\text { TI } \\
(\mathrm{ms})\end{array}$ & $\begin{array}{c}\text { Matrix } \\
\text { Freq*phase }^{*}\end{array}$ \\
\hline Loc(Sag) & 25 & 14 & 5 & 10,000 & 73 & - & $128 * 128$ \\
\hline DWI(Ax) & 25 & 14 & 5 & 10,000 & 73 & - & $128 * 128$ \\
\hline FLAIR(Ax) & 25 & 14 & 5 & 11,000 & 170 & 2250 & $256 * 192$ \\
\hline$T_{2}(A x)$ & 25 & 14 & 5 & 5,000 & 102 & - & $256 * 256$ \\
\hline $\begin{array}{c}\text { 3D } \\
\text { SPGR(Ax) }\end{array}$ & 25 & 14 & 5 & 7.5 & 7.5 & - & $256 * 192$ \\
\hline Perf(EPI) & 25 & 14 & 5 & 2000 & 20 & - & $128 * 128$ \\
\hline $\begin{array}{c}\text { 3D } \\
\text { SPGRGD(Ax) }\end{array}$ & 25 & 14 & 5 & 7.5 & 7.5 & - & $256 * 192$ \\
\hline $\mathrm{T}_{1} \mathrm{GD}(\mathrm{Ax})$ & 25 & 14 & 5 & 750 & 7.5 & - & $256 * 224$ \\
\hline
\end{tabular}

The obtained images were preprocessed before segmentation. The images were first converted into 3D datasets using Analyses of Functional Neuroimages (AFNI) software $^{40}$. Since structure of the human brain varies across subjects, the first step for each subject was to align individual MR image scans. The subjects head position may not be in the same location during each and every scan taken. Also, image distortion varies from pulse sequence to pulse sequence.

Most brain atlases are based on a detailed representation of anatomy in a standardized 3D coordinate system, or stereotaxic space. Anatomical points are chosen on high resolution $\left(2 \mathrm{~mm}^{3}\right)$ 3D anatomical data sets. Anatomical markers help in transforming the MR data 
sets to stereotaxic space. The stereotaxic coordinate system ${ }^{41}$ is defined as below:

The anatomical landmarks are:

- Anterior commissure (AC) - It is a thin white matter tract connecting each hemisphere near the olfactory areas of the brain.

- Posterior commissure (PC) - It is a C-shaped structure slightly rotated in a clock-wise direction located posterior and inferior to the AC also connects the hemispheres.

There are two nerve fibers connecting the cerebral hemispheres across the longitudinal fissure as shown in Figure 3.5.

- The anterior-to-posterior horizontal axis (y) is the tangent to the superior edge of the AC and to the inferior edge of the PC, at points crossing the longitudinal fissure.

- The vertical axis (z) is perpendicular to the AC-PC line, in the longitudinal fissure, and the tangent to the posterior edge of the AC.

- The remaining axis (right-to-left or $\mathrm{x}$ ) is perpendicular to the $\mathrm{y}$ and $\mathrm{z}$ axes.

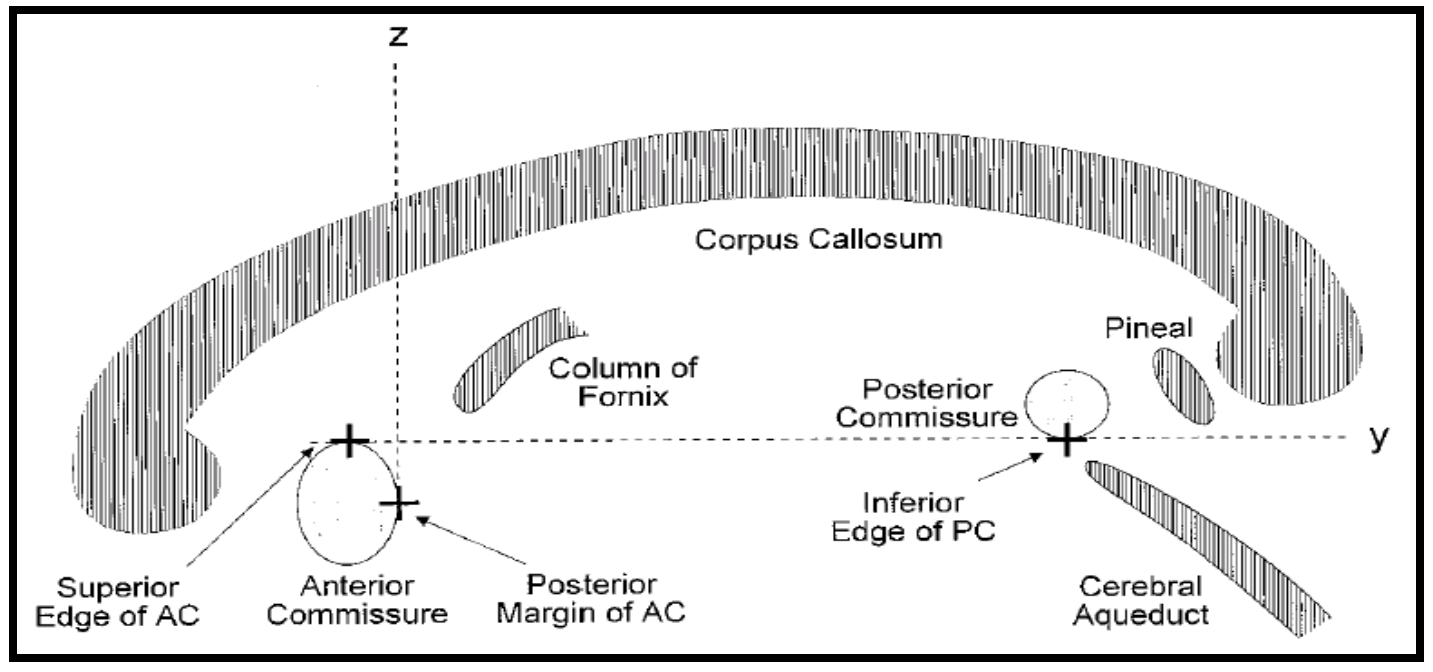

Figure 3.5 Sketch of the central portion of the midline of the brain showing anatomical landmarks used to specify the stereotaxic coordinate system.

The transformation to the AC-PC-aligned view was made by placing "markers" as shown in Figure 3.5 on:

- The superior and posterior edges of the AC.

- The inferior edge of the PC.

- Given the AC-PC line (the new y-axis), one point in the new yz plane. 
- Two points (separated by at least $20 \mathrm{~mm}$ ).

After choosing anatomical landmarks, the whole brain volume was rotated to align with the stereotaxic axes. This aligned, not scaled, coordinate system data set is called the AC-PC aligned view. The aligned datasets are then resampled into $1 \times 1 \times 1 \mathrm{~mm}^{3}$ resolution in the slice select direction between input slices using cubic interpolation. The aligned data sets were written out as ACPC aligned volumes along with a header file.

Another method for co-registering the data sets of the same subject is using rigid body transformation in SPM2 software ${ }^{42}$. SPM contains a list of subroutines based on MATLAB. Rigid body transformation is a subset of the affine transformations. Affine transformation is described by six parameters, three translational and three rotations along the orthogonal axes. For each point in an image $\left(\mathrm{x}_{1}, \mathrm{x}_{2}, \mathrm{x}_{3}\right)$, an affine mapping is defined into the co-ordinates of another space $\left(\mathrm{y}_{1}, \mathrm{y}_{2}, \mathrm{y}_{3}\right)$. The mapping is described as a simple matrix multiplication:

$$
\mathrm{y}=\mathrm{Mx}
$$

Where, $\mathrm{x}$ - matrix containing values of an image, $\mathrm{y}$ - matrix containing co-ordinates of another space for the corresponding values of $\mathrm{x}$, $\mathrm{M}$ - matrix containing mapping values.

Using SPM2 software to co-register the images, an affine transformation was applied in which one of the images was considered as a reference image (the target image usually SPGR image). Another image was transformed (mapped) to match the co-ordinates of the target image. The images are then resampled based on the transformations by trilinear interpolation method. The voxel dimensions are resampled to the SPGR image voxel size. Hence, each image type ( $T_{1}, T_{2}$, FLAIR etc.) are mapped to the SPGR image using an affine transformation. The images are then spatially normalized where each subject's image is mapped to a standard space. This allows for averaging of data and comparison between subjects. The normalization is usually done by spatially transforming the images to a template image in standard space. The sum of the squared differences between the template image and the image is minimized or the correlation between the images is maximized. Spatial normalization can also be carried out using affine transformation. 
This transformation uses a 12 parameter rigid body nonlinear transformation to map the image to a template. The intensity of the images may be scaled and smoothed to a certain extent in this method.

Next, the high resolution SPGR images are segmented into gray matter (GM), white matter (WM) and Cerebrospinal fluid (CSF). The segmentation using SPM is based on the maximum likelihood mixture model clustering algorithm. It utilizes a priori probability images of GM, WM, and CSF ${ }^{43}$. This model assumes that MR images contain a certain number of tissue types from which every voxel has been drawn. Through spatial transformation each voxel from the high resolution images was mapped to the corresponding location in the a priori probability images. The mapping was achieved by least squares matching the template images in the same standard space. Estimating which voxel belongs to which tissue type requires an iteration process. The iterative process ends when the change in log-likelihood function is negligible. The algorithm is also known as the expectation maximation (EM) algorithm ${ }^{43}$. The E-step denotes the calculation of probabilities of belonging to a cluster and the M-step computes the clusters and non-uniformity correction parameters. For the calculation of probabilities belonging to a cluster, Bayes rule ${ }^{44}$ is used. For non-uniformity correction parameters, discrete cosine transform is utilized. SPM fails when patient data are analyzed as there are no prior probability images that can be used as a template image.

In order for the segmentation to start, the AFNI data sets were first converted to ANALYZE format for compatible use in SPM2 where by the AFNI data sets were converted to ".img" format along with a header file. All the images were co-registered, spatially normalized and resampled to $1 \mathrm{x} 1 \mathrm{x} 1 \mathrm{~mm}^{3}$ resolution. The ".img” files were then read into a MATLAB script for performing fuzzy c-means segmentation of tissue types. Similarly, using SPM2 software, the high resolution SPGR images alone were segmented into the three tissue types (GM, WM, and CSF). The segmentation results using fuzzy cmeans clustering were then compared to the segmentation results using SPM2. 


\section{Chapter 4}

\section{Results}

\section{Simulation Using Stripes}

The FCM MATLAB code was executed for the simulation of stripes which contained seven tissue types. The stripe model shown below contains $\mathrm{T}_{1}, \mathrm{~T}_{2}$, FLAIR intensity values with no noise.

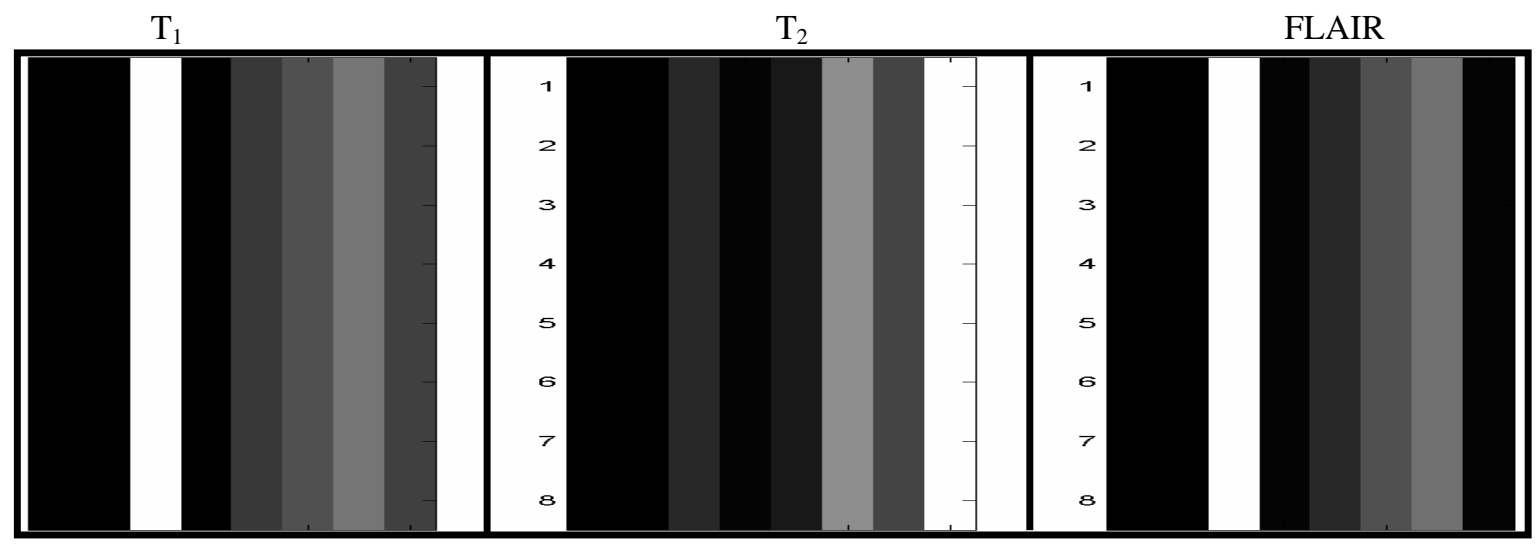

Figure 4.1 Simulation stripes of seven tissues with three types of images.

The eight tissues in Figure 4.1 are: air, air, scalp, skull, meninges, gray matter, white matter, CSF. The air tissue is repeated twice in order to form an 8 x 8 stripe. The number of classes was chosen as seven for FCM segmentation and they were executed for $\mathrm{m}$ values of 1.05, 1.25, 1.5, 1.75, 2.0, noise levels of 0, 10, 20 (arbitrary units) and threshold values of $0.55,0.75,0.95$. Since the number of classes was seven, each class (tissue) is assigned a color from the color map. The color map designed is shown below:

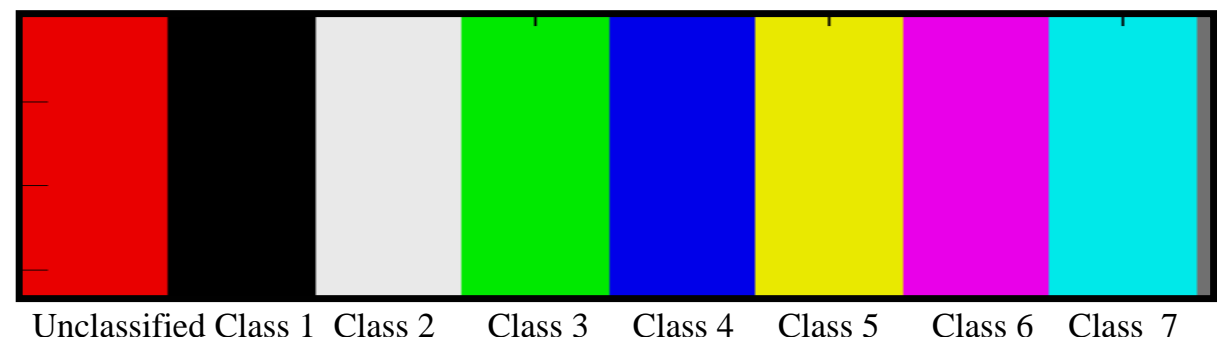

Figure 4.2 Color map used for normal subjects. 


\section{Cluster center plots:}

The segmentation was carried out for feature vectors composed of pixels from $T_{1}, T_{2}$ and FLAIR images. The cluster centers in 3-D space of the respective classes are plotted for each feature vector. The plots help in determining the number of classes (tissues) to be used. Shown below is a cluster center plot for $\mathrm{m}=2.0$, threshold $=0.55$.

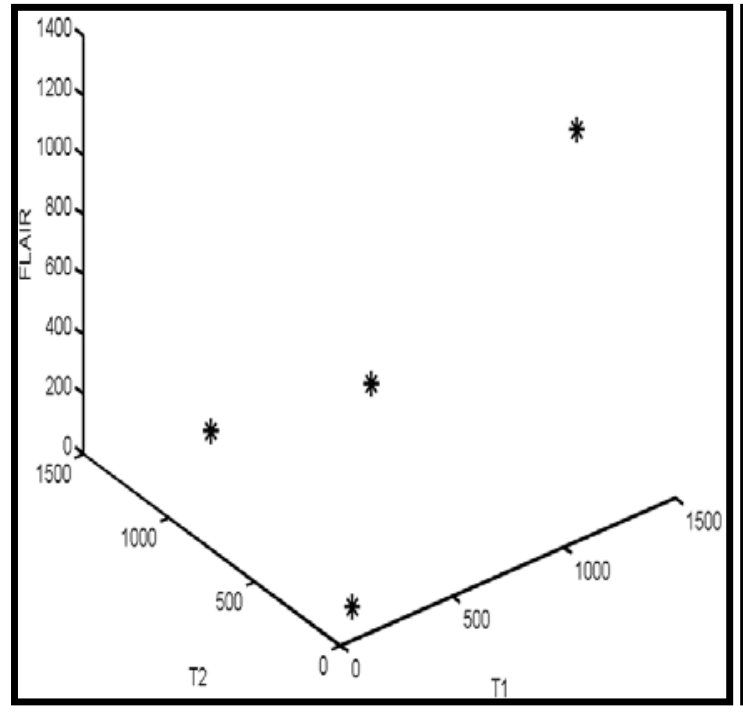

Figure 4.3 Cluster center plots a) Clusters $=4$

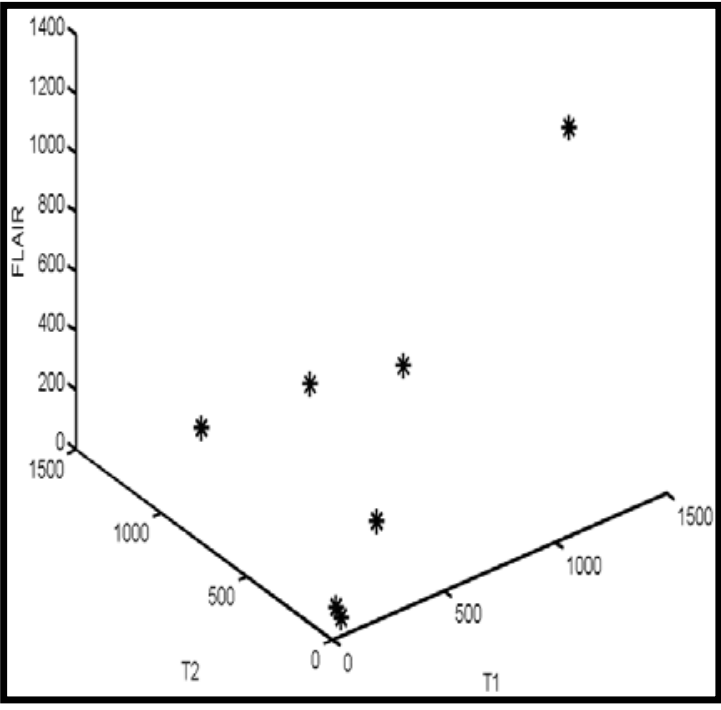

b) Cluster $=7$

Figure 4.3 shows the difference in cluster centers when we segment the data into cluster $=4$ and cluster $=7$. Note that when we group them into a larger number of clusters the centers are closer to each other thereby segmenting the data differently. Also, since the intensity values of air and scalp are close to each other, their respective cluster centers in Figure $4.3 \mathrm{~b}$ close to the origin can be seen to be redundant.

\section{Membership degree plots:}

During the iteration of the algorithm, the membership value are determined and updated. The membership values for each class were plotted. These plots help in determining the $\mathrm{m}$ (fuzziness) for the data set and also in setting the threshold. The membership values for $\mathrm{m}=1.25$ for two different classes are shown in Figure 4.4. 


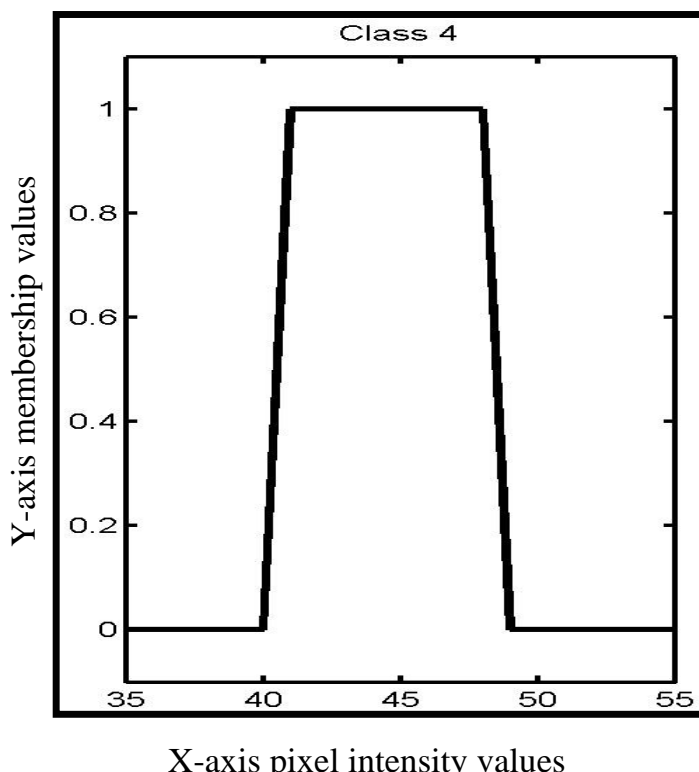

Figure 4.4 Membership value plot

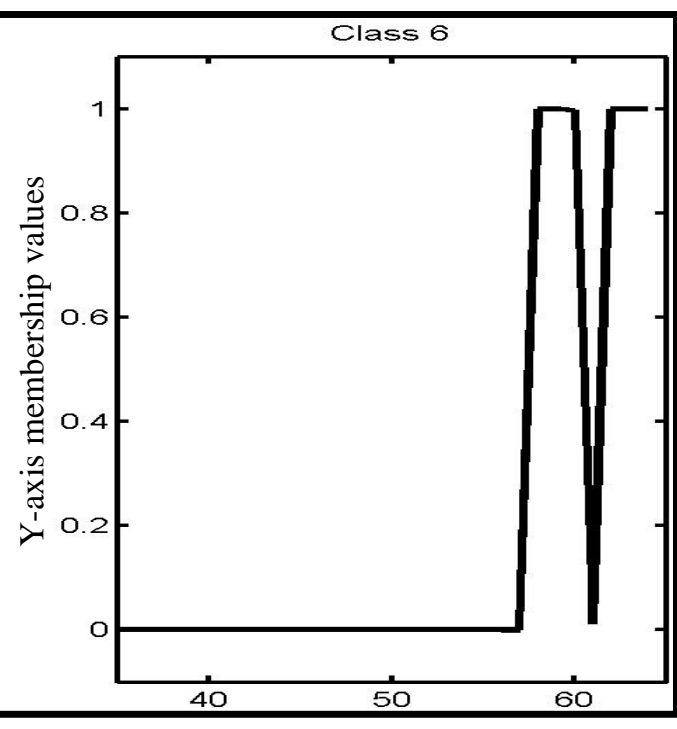

$\mathrm{X}$-axis pixel intensity values
a) class - 4
b) class - 6

Figure 4.4a shows that the membership degrees range between 0 and 1 for the pixel intensities from 35 - 55. Figure 4.4b shows that in the range of 35-55 pixel intensities the membership degree is zero. This indicates that pixel values in that range do not belong to cluster 4 . The membership value plots for $m=2.0$ is shown below.

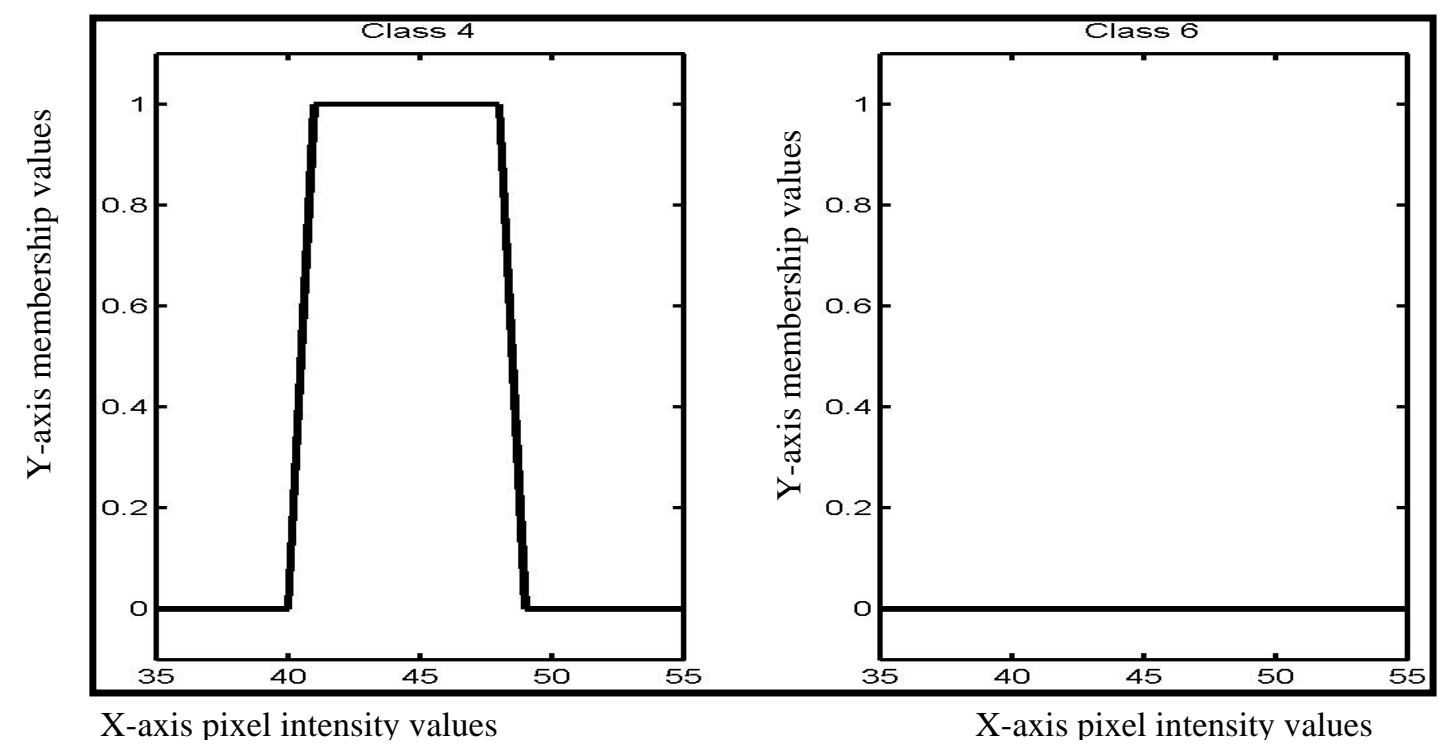

Figure 4.5 Membership value plots a) class - $4 \quad$ b) class - 6 
The membership values for the same pixel range as in Figure 4.4 for the class 6 is zero in Figure 4.5. Using these plots the $\mathrm{m}$ value was fixed at 1.5 with number of classes $=7$.

\section{Threshold value:}

The threshold value determines which pixels belong to a particular (class) based on its maximum membership value. Since each stripe is a tissue, perfect segmentation should result in each color stripe only. The tissue class containing a mixture of colors may represent that the tissue class may contain two or more tissues. The simulated segmented images of $\mathrm{T}_{1}, \mathrm{~T} 2$, and FLAIR for noise $=0, \mathrm{~m}=1.5$, threshold $=0.55$ are shown below in Figure 4.6.

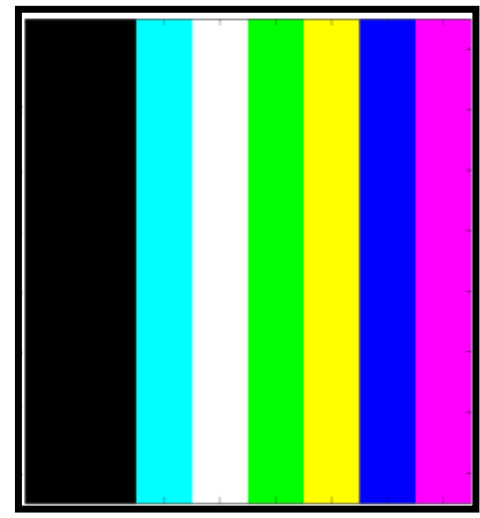

a) Threshold value $=0.55$

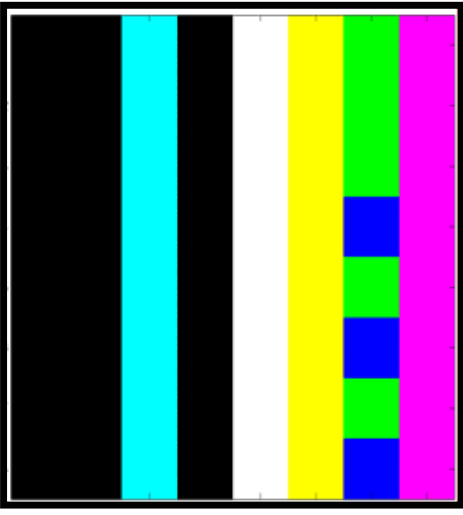

b) Threshold value $=0.75$

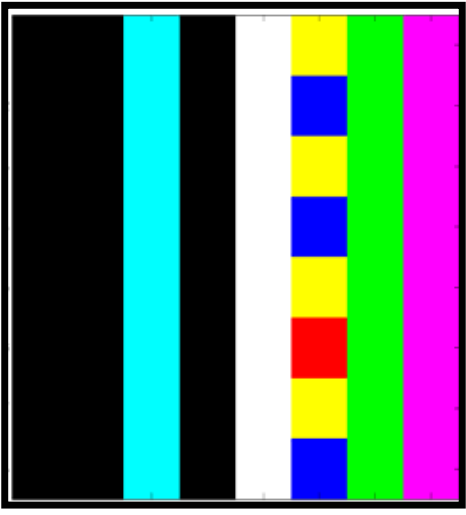

c) Threshold value $=0.95$

Figure 4.6 FCM simulated images for noise $=0$.

The differences in threshold values for a fully segmented and mixed segmentation class are shown below in Figure 4.7.

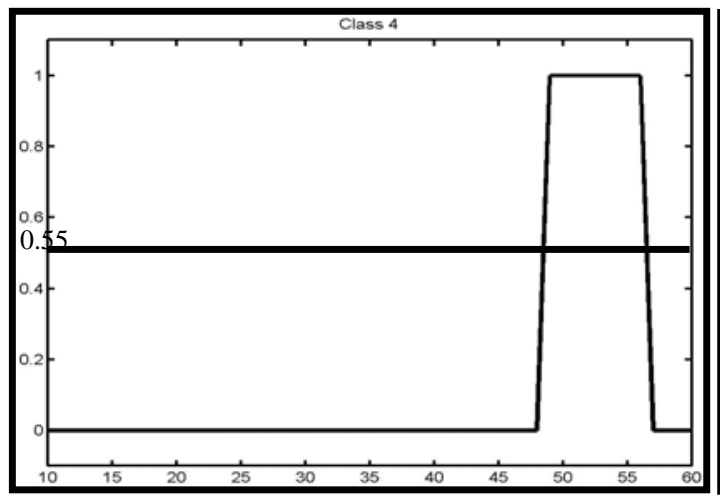

a) Pure segmentation class 4

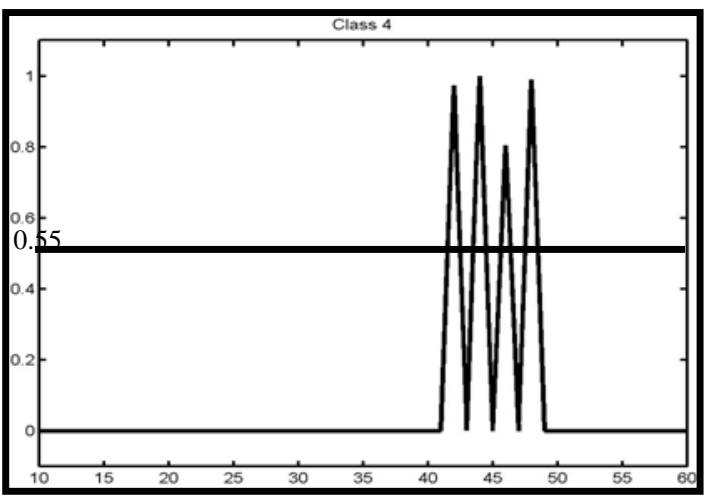

b) mixed segmentation class 4

Figure 4.7 Membership value plot showing threshold $=0.55$. 
From Figure 4.6 and 4.7 it can be seen that as the values of threshold increases mixed segmentation results. In the case where threshold value is less than 0.55 , again mixed segmentation was obtained. Hence for our data set an optimized threshold value of 0.55 was chosen. The simulated segmented images of $T_{1}, T 2$, and FLAIR for noise $=10$, $\mathrm{m}=1.5$, threshold $=0.55$ are shown in Figure 4.8.

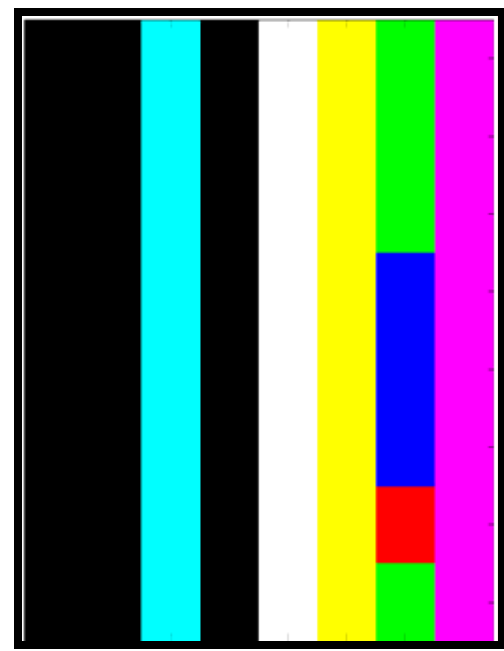

a) Threshold value $=0.55$

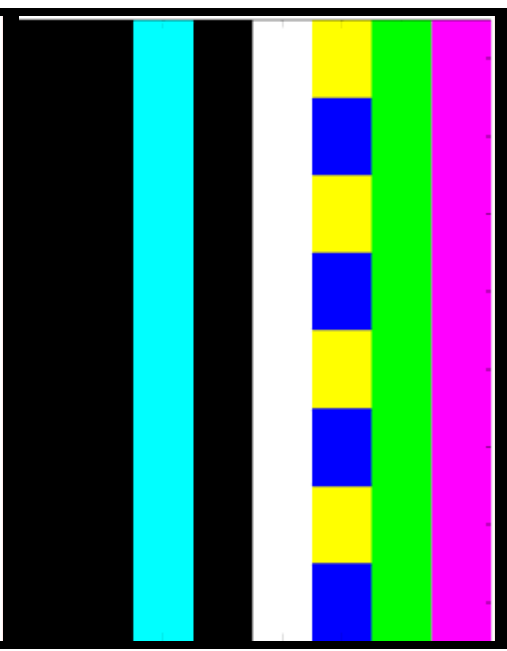

b) Threshold value $=0.75$

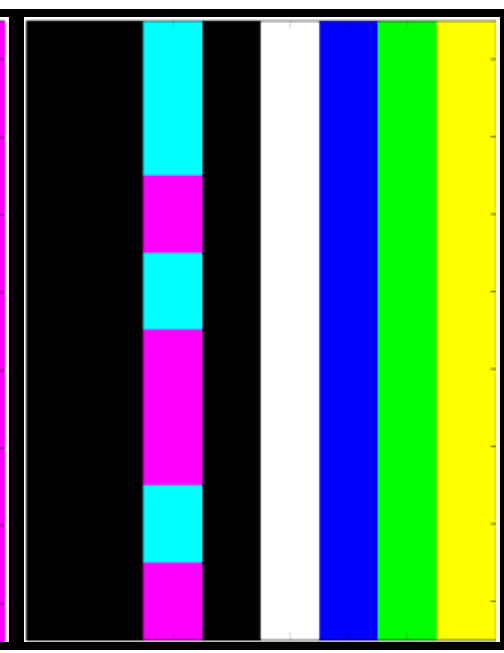

c) Threshold value $=0.95$

Figure 4.8 FCM simulated images for noise $=10$.

\section{Partial Volume Stripes}

As described in chapter 3, a partial volume stripe is created for the three feature vector ( $\mathrm{T}_{1}, \mathrm{~T}_{2}$, and FLAIR) as shown in Figure 4.9.

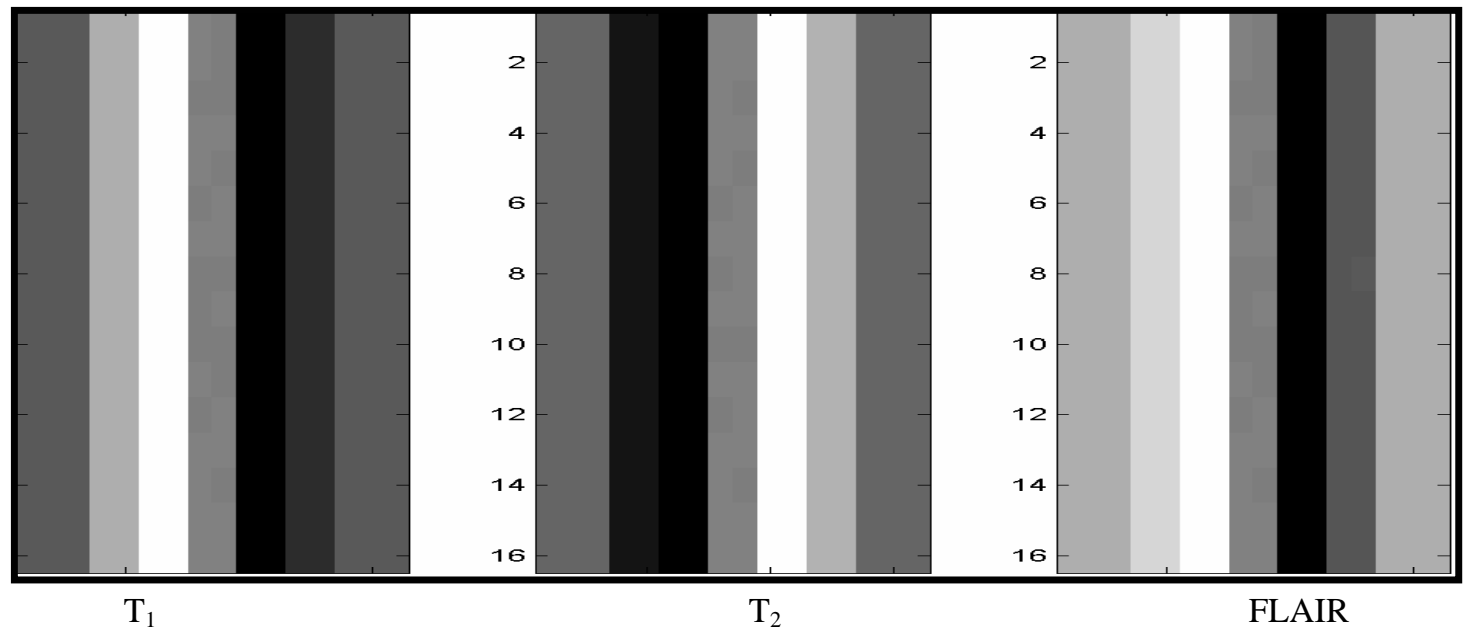

Figure 4.9 Partial volume simulation stripe. 
These simulation stripes were executed for $m$ values in the range of ima and a threshold value of $0.55,0.75$ and 0.95 with different noise levels. Figure 4.10 is a FCM segmented simulation stripe with different threshold values for an $\mathrm{m}=1.5$.

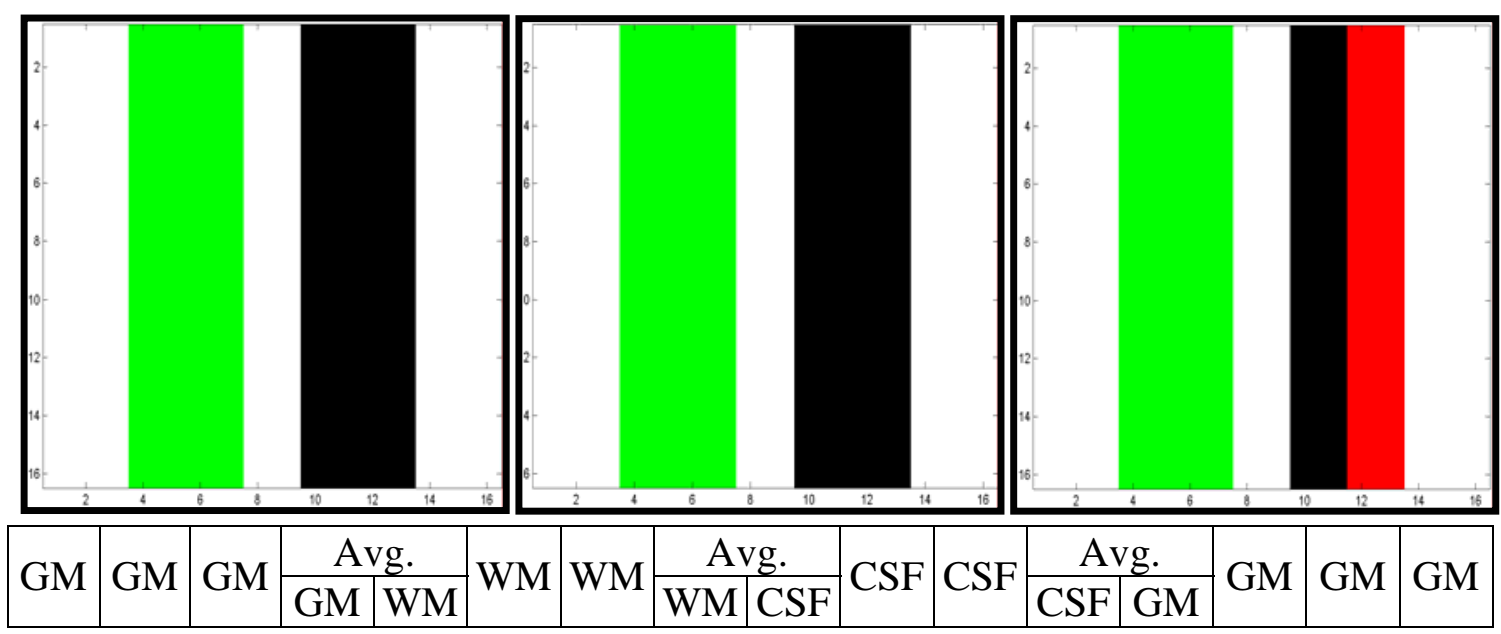

Figure 4.10 FCM segmented images for partial volume simulation.

The red pixels in Figure 4.10 c) indicate unclassified tissue type. In Figure 4.10 a) the pixel stripe containing a mixture of white matter and CSF is classified as gray matter. The stripes may indicate more weighting to white matter. The cluster centers and membership degrees are also plotted for this simulation stripe.

\section{Classification error:}

The cluster plots and membership degrees for both types of simulation stripes change for different threshold values for different noise levels. The percentage error of misclassified pixels is calculated as:

(Number of incorrect pixels / Total number of pixels) x 100

These values help in determining the range of $\mathrm{m}$ values and threshold values that can be used for segmenting our MRI data set.

\section{Brain ROI Simulation Model}

A region of interest was selected from the normal subject's MRI data set as described in chapter 3 . The region of interest contained only three types of tissue - GM, WM, CSF. The image vectors included were FLAIR, $\mathrm{T}_{2}$, SPGR and FCM algorithm is executed for 
class $=3$ for the ROI alone. The image inputs to FCM are shown in Figure 4.11.
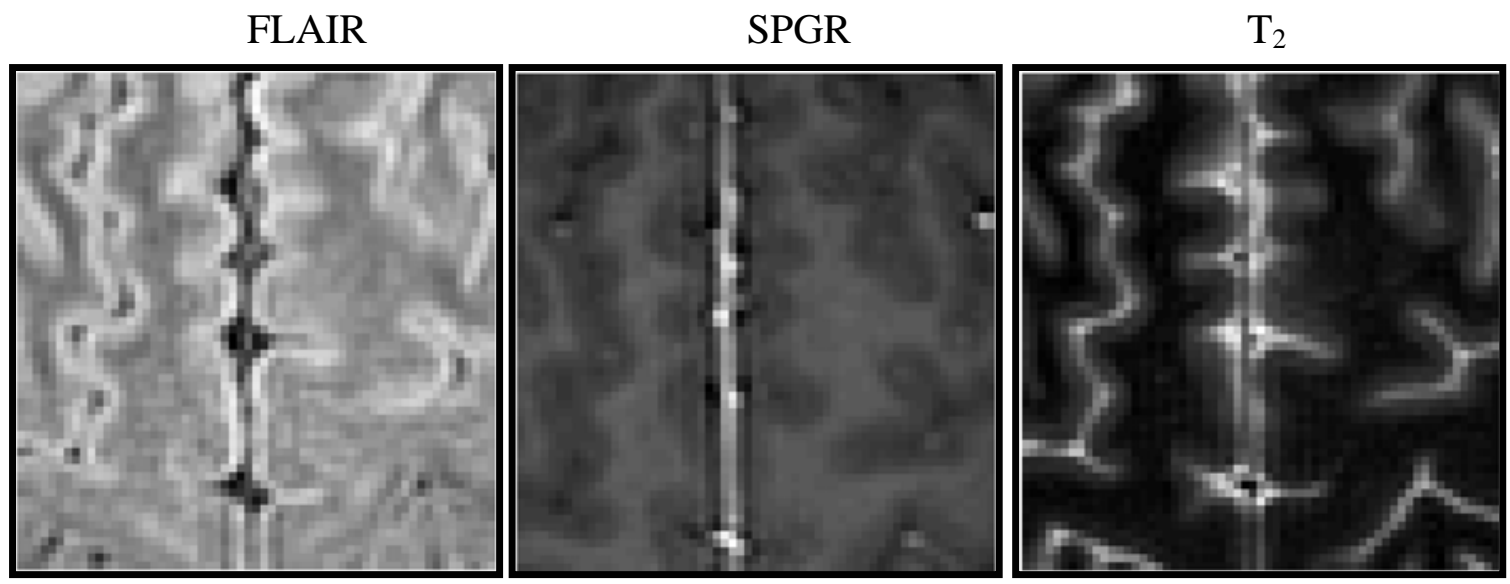

Figure 4.11 Brain ROI of three image vectors

The FCM was executed for different $m$ values and thresholds of $0.55,0.75$ and 0.95 . The FCM segmented image for $m=1.5$ is shown below in Figure 4.12.

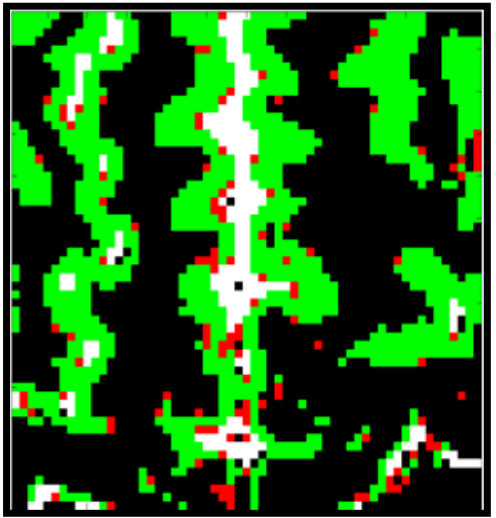

a) Threshold $=0.55$

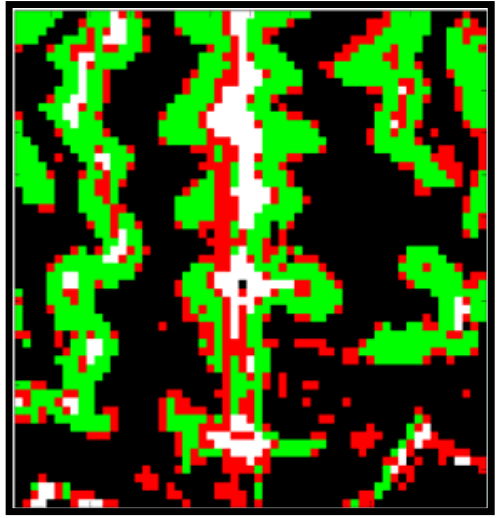

b) Threshold $=0.75$

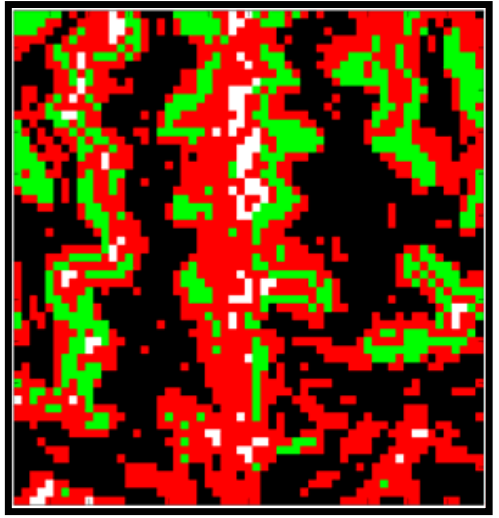

c) Threshold $=0.95$

Figure 4.12 FCM segmented Brain ROI, Green: Gray Matter; Black: White matter;

White: CSF, Red: unclassified.

Comparing Figure 4.12 a) segmented image to the original images, the segmentation is good. Though there might be some more weighing towards white matter consistent with the partial volume stripe model. From the above images it is seen that as the threshold value increases the number of unclassified pixels increases. Hence for our data set, $\mathrm{m}=$ 1.5 with threshold $=0.55$ is chosen as the optimal value for MR whole volume data sets. 


\section{MRI Data Sets}

The whole volume MRI data sets are then segmented using FCM for each subject. The optimal values are fixed at $\mathrm{m}=1.5$, threshold $=0.55$. Figure 4.13 shows the FCM segmented image for a normal subject for feature vectors (FLAIR, $T_{2}$, SPGR pre and post contrast, DWI). The feature vector is segmented into six tissue types for normals and nine tissue types for patients with tumor. Different colors from the color map represent different tissue types. The FCM segmented image when another feature vector DWI is added is also shown in Figure 4.13.

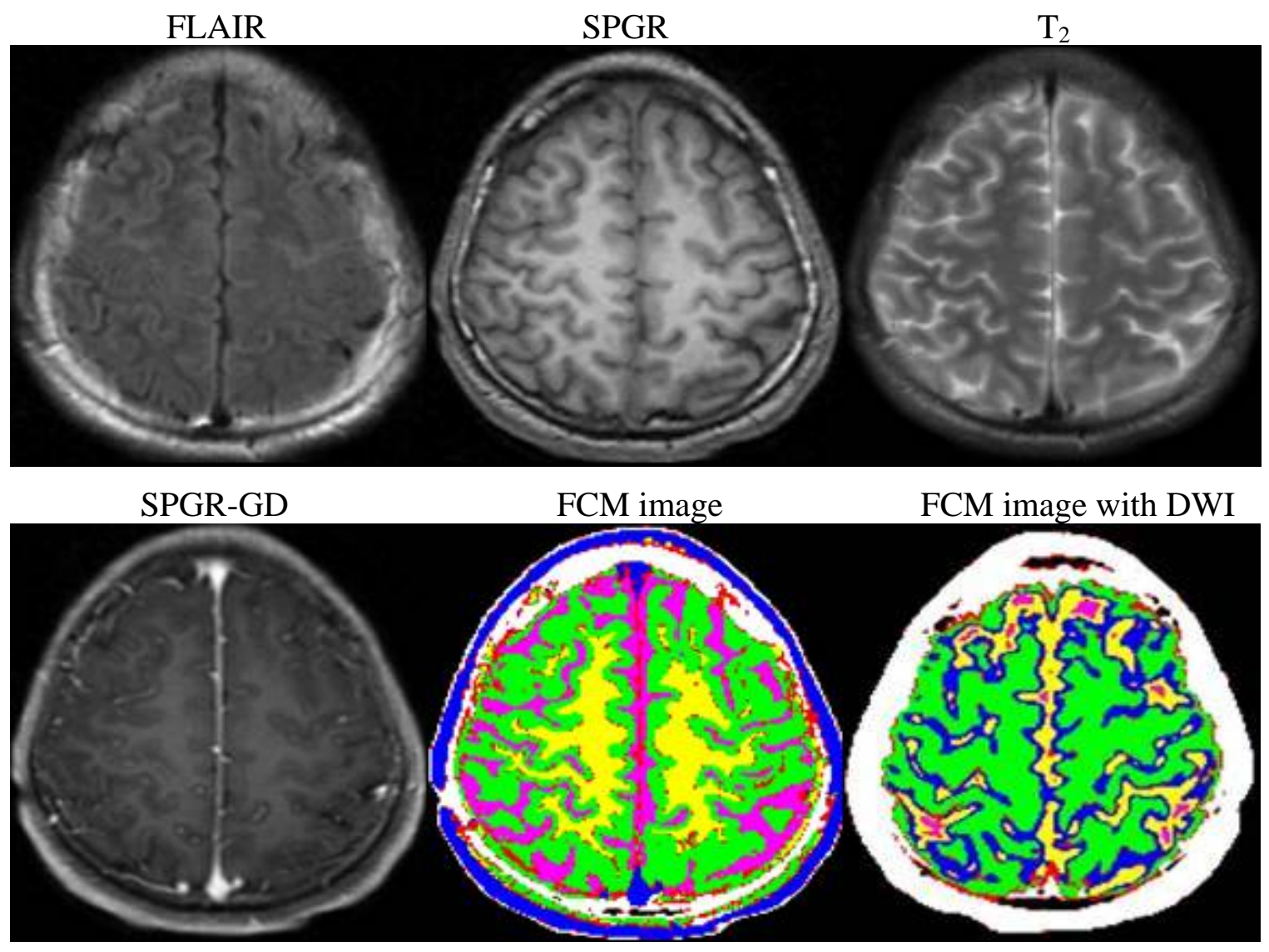

Figure 4.13 FCM segmentation of normal subject.

The class representing gray matter (GM), white matter (WM), and CSF are extracted from the single colored image and displayed separately in Figure 4.14 a). GM, WM, CSF maps are obtained from the standard SPM software and displayed in Figure 4.14 b). FCM images are subtracted from the SPM maps and the difference maps are displayed in Figure 4.14 c). 


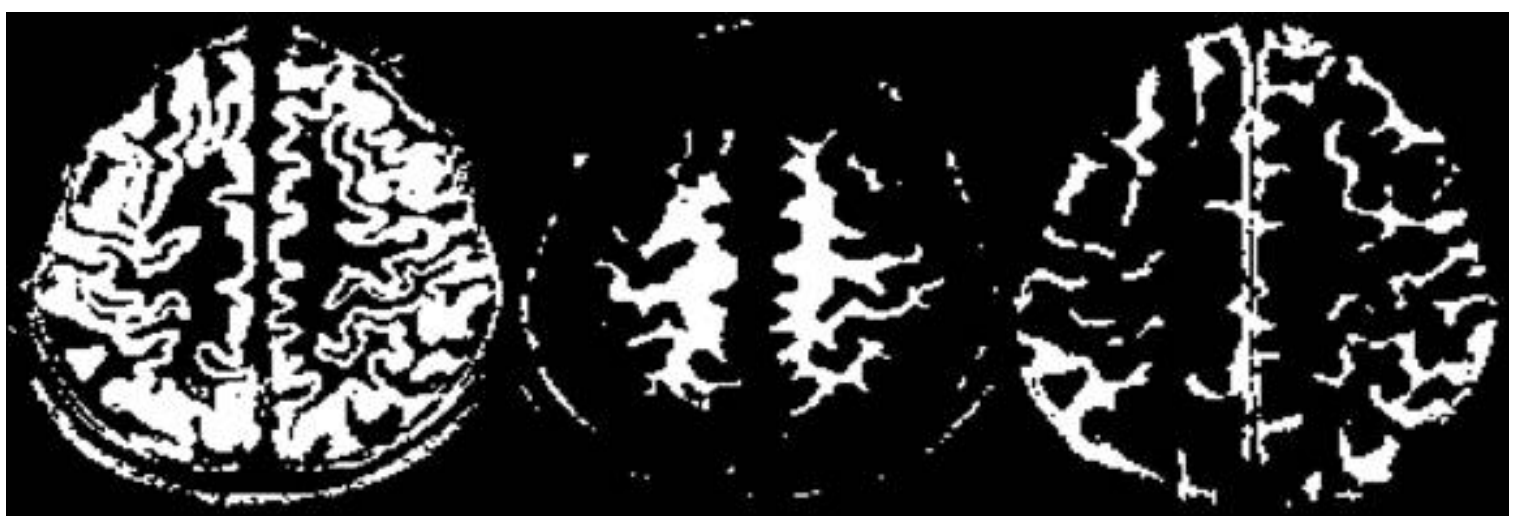

a) FCM: Gray Matter

White Matter

CSF

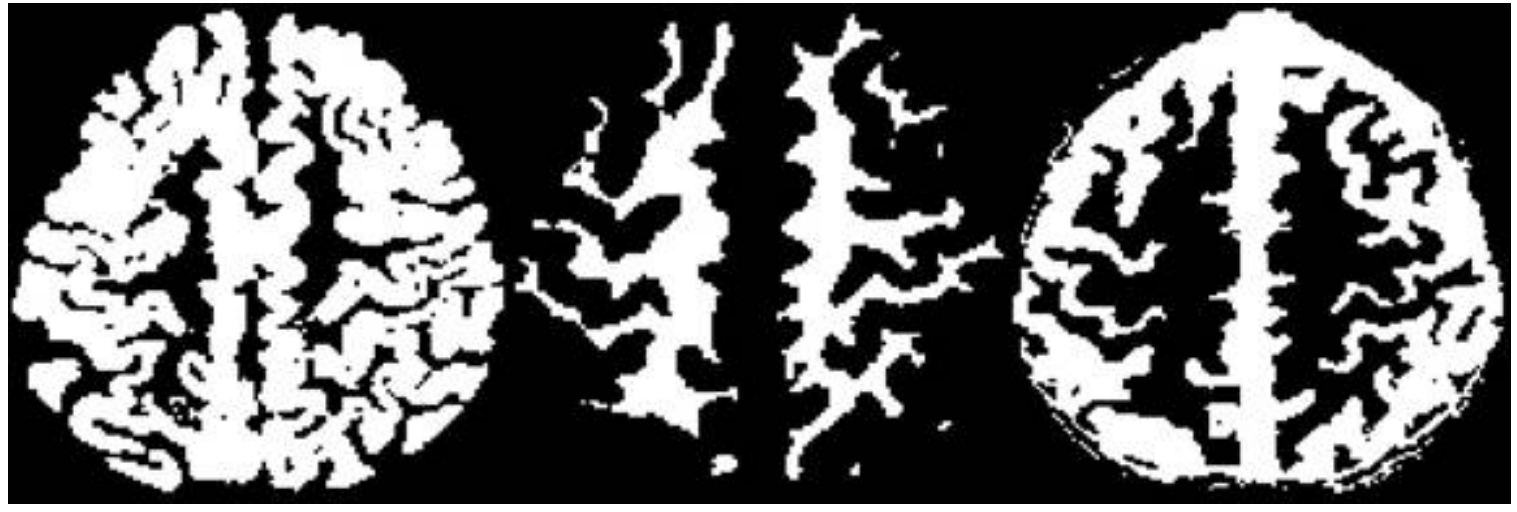

b) SPM: Gray Matter

White Matter

CSF

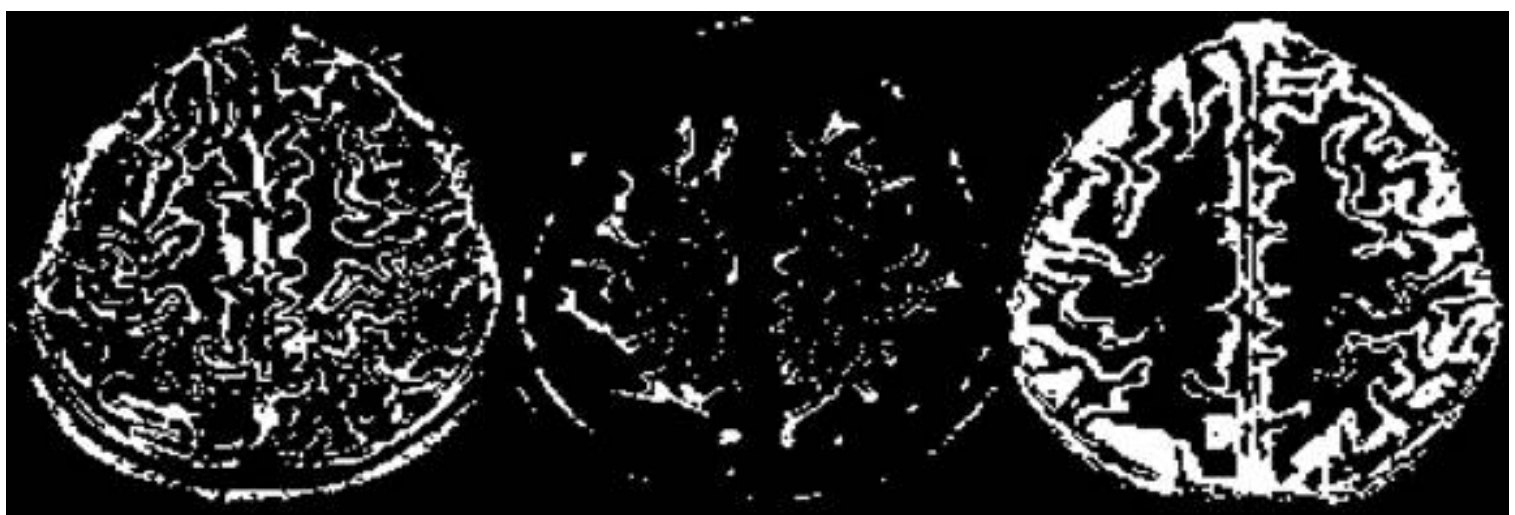

c) Difference image: Gray Matter White Matter

CSF

Figure 4.14 Difference maps of normal subject

For a tumor patient, as seen in Figure 4.15 the feature vector (FLAIR, $\mathrm{T}_{2}, \mathrm{SPGR}$, SPGRGD, DWI) is segmented into nine tissue types. This is done in order to include the tumor tissues, edema and necrosis. Figure 4.15 also shows the FCM segmented image of tumor subject with the addition of DWI to the feature vector. 

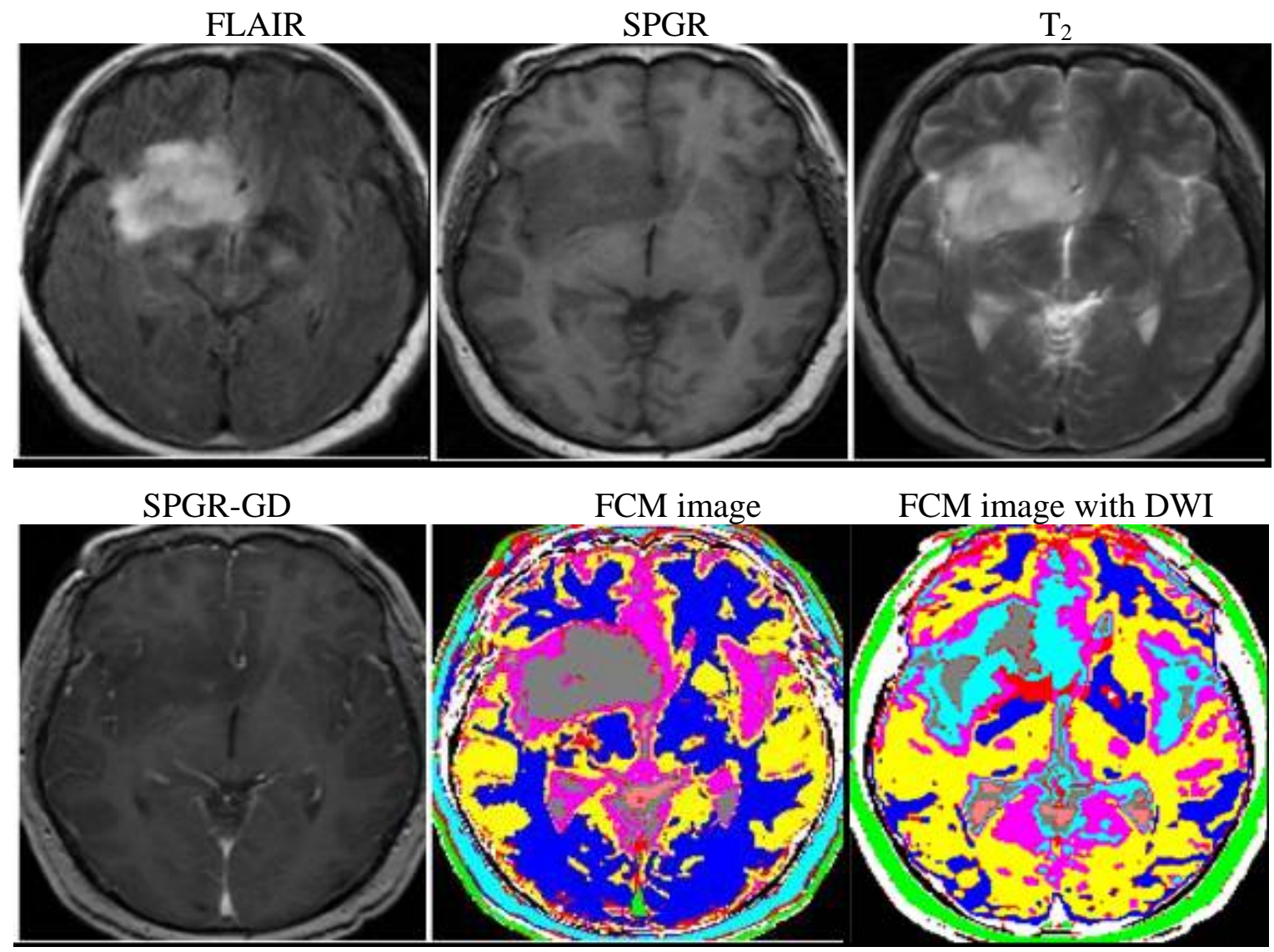

Figure 4.15 FCM segmentation of tumor patient.

The class representing gray matter (GM), white matter (WM), and CSF for tumor subject are extracted from the single colored image and displayed separately in Figure $4.16 \mathrm{a}$ ). GM, WM, CSF maps are obtained from the standard SPM software and displayed in Figure 4.16 b). FCM images are subtracted from the SPM maps and the difference maps are displayed in Figure $4.16 \mathrm{c}$ ). The results for all the subjects are shown in Appendix A. The percentage of difference for the maps are calculated and tabulated in Table 6 and 7 with and without DWI respectively. 


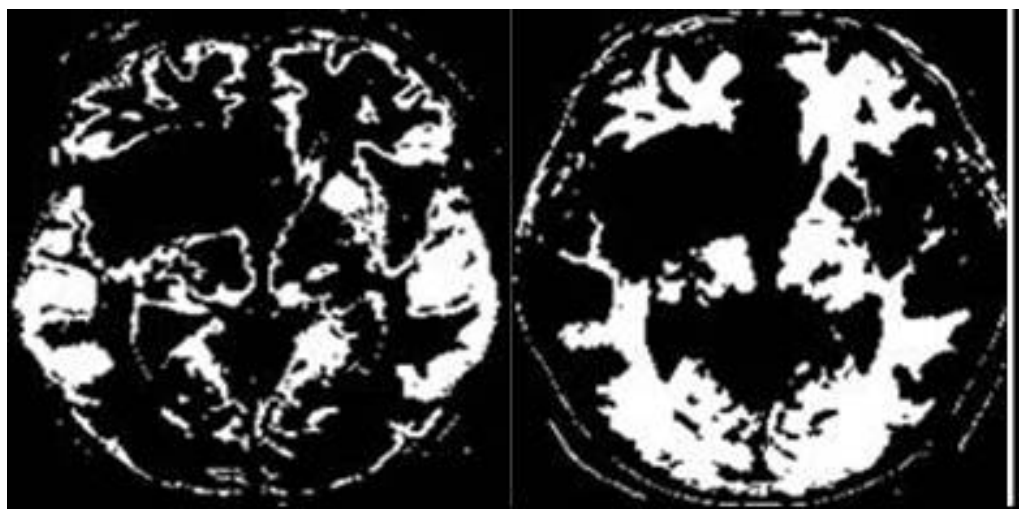

a) FCM segmented GM map

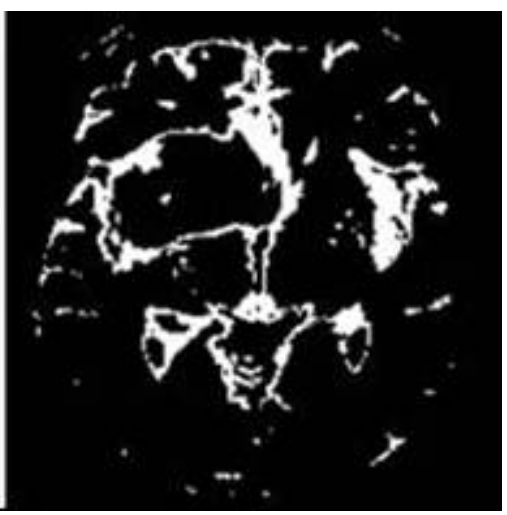

CSF map

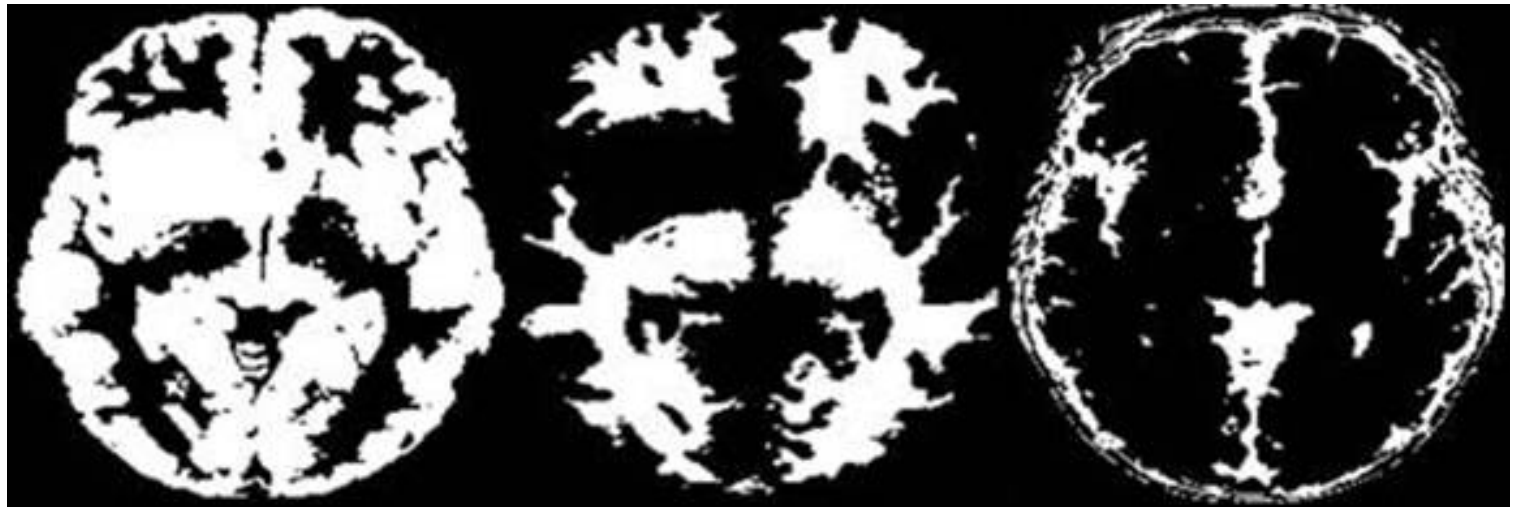

b) SPM segmented GM map WM map

CSF map

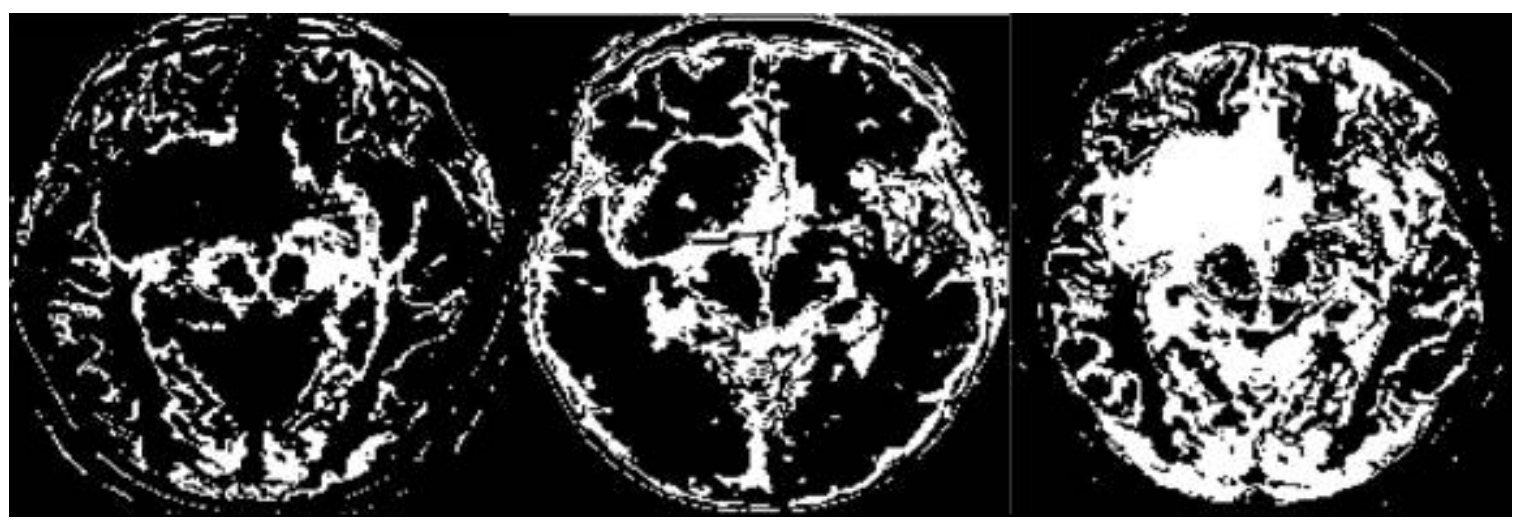

c) Difference maps GM map

WM map

CSF map

Figure 4.16 Difference maps of tumor patient. 
Table 6: Quantitative Difference in GM, WM, CSF maps using four feature vectors.

\begin{tabular}{|c|c|c|c|c|c|c|c|c|c|}
\hline \multirow{2}{*}{ Subjects } & \multicolumn{3}{|c|}{ Gray matter } & \multicolumn{3}{|c|}{ White matter } & \multicolumn{3}{|c|}{ CSF } \\
\hline & FCM & SPM & difference & FCM & SPM & difference & FCM & SPM & difference \\
\hline \multicolumn{10}{|c|}{ Normal subj: } \\
\hline N1 & 23.54 & 37.08 & 24.73 & 21.51 & 24.21 & 11.16 & 1.90 & 17.56 & 15.85 \\
\hline N2 & 26.36 & 35.25 & 15.30 & 22.79 & 23.92 & 4.88 & 8.37 & 20.85 & 14.23 \\
\hline N3 & 24.18 & 34.10 & 16.24 & 23.20 & 27.28 & 7.15 & 8.55 & 16.58 & 13.46 \\
\hline N4 & 20.85 & 36.77 & 17.69 & 26.77 & 37.46 & 11.45 & 4.28 & 11.83 & 16.11 \\
\hline \multicolumn{10}{|c|}{ Tumor subj: } \\
\hline $\mathrm{P} 1$ & 18.50 & 36.30 & 24.93 & 14.73 & 22.51 & 14.95 & 3.66 & 21.08 & 17.76 \\
\hline $\mathrm{P} 2$ & 11.46 & 36.53 & 29.87 & 15.51 & 28.54 & 25.54 & 3.40 & 18.37 & 15.94 \\
\hline P3 & 19.07 & 37.63 & 24.32 & 19.73 & 21.89 & 11.23 & 10.70 & 18.90 & 22.08 \\
\hline $\mathrm{P} 4$ & 17.59 & 36.98 & 23.15 & 18.32 & 23.14 & 11.08 & 8.34 & 12.04 & 16.95 \\
\hline
\end{tabular}

Table 7: Quantitative Difference in GM, WM, CSF maps using five feature vectors.

\begin{tabular}{|c|c|c|c|c|c|c|c|c|c|c|}
\hline \multirow{2}{*}{ Subjects } & \multirow{2}{*}{ \# slices } & \multicolumn{3}{|c|}{ Gray matter } & \multicolumn{3}{|c|}{ White matter } & \multicolumn{3}{|c|}{ CSF } \\
\hline & & FCM & SPM & difference & FCM & SPM & difference & FCM & SPM & difference \\
\hline \multicolumn{11}{|l|}{$\begin{array}{l}\text { Normal } \\
\text { subj: }\end{array}$} \\
\hline N1 & 70 & 21.18 & 35.48 & 21.43 & 20.53 & 23.85 & 6.90 & 10.59 & 17.92 & 18.64 \\
\hline $\mathrm{N} 2$ & 55 & 19.00 & 35.04 & 21.62 & 29.38 & 23.61 & 12.34 & 1.23 & 20.88 & 19.76 \\
\hline N3 & 39 & 17.31 & 33.93 & 21.87 & 34.23 & 26.79 & 15.54 & 3.83 & 16.66 & 15.34 \\
\hline $\mathrm{N} 4$ & 27 & 20.64 & 45.67 & 37.32 & 32.39 & 30.71 & 15.47 & 1.90 & 10.41 & 10.05 \\
\hline \multicolumn{11}{|c|}{ Tumor subj: } \\
\hline $\mathrm{P} 1$ & 63 & 8.83 & 36.28 & 31.64 & 15.44 & 27.11 & 13.14 & 2.55 & 20.57 & 18.39 \\
\hline $\mathrm{P} 2$ & 76 & 14.04 & 38.11 & 37.77 & 15.36 & 29.45 & 24.93 & 2.06 & 18.55 & 17.40 \\
\hline P3 & 50 & 14.38 & 38.31 & 29.06 & 23.51 & 23.41 & 17.27 & 2.70 & 18.91 & 18.31 \\
\hline
\end{tabular}




\section{Chapter 5}

\section{Discussion and Conclusions}

In this thesis, unsupervised fuzzy clustering was applied to MRI multispectral data $\left(\mathrm{T}_{2}\right.$, FLAIR, SPGR, SPGRGD, DWI) for segmenting two types of images: those from normal brain and from brain with brain tumor. Our hypothesis was that using different MRI image types instead of the previously used $\mathrm{T}_{1}$-wtd., $\mathrm{T}_{2}$-wtd., and proton density MRI with FCM could yield better segmentation results for brain MRI of patients with brain tumor(s). We used four-dimensional feature vectors composed of FLAIR, $\mathrm{T}_{2}$-wtd., SPGR, and SPGR post Gd-contrast administration. Each voxel contained data for a single pixel from each of the scans. We also added another dimension to the feature vectors by including DWI-MRI. This extension to more and differently composed feature vectors has been suggested in previous work ${ }^{39}$.

To the best of our knowledge, our work is the first whole-brain fully automated multi tissue segmentation that has been performed on both normal and patient data sets. The inclusion of the whole cerebrum provides more of each tissue type which improved the segmentation for the patient data. One drawback of previously published single slice segmentation is that it cannot be as automated since each slice may contain different numbers of tissue types as the location changes. All tissue types were always included in this FCM processing so the number of classes remains stable. The data sets were checked for registration and then resliced to $1 \mathrm{~mm} \times 1 \mathrm{~mm} \times 1 \mathrm{~mm}$ voxels for the segmentation. These results are not presented here. Having performed the appropriate and necessary preprocessing we could then quantify the difference between segmentation by our routine and another routine for the gray matter volume, the white matter volume and CSF volumes. We compared FCM to another brain segmentation package, SPM2 ${ }^{27}$. To our knowledge quantification by difference images of this sort has not been presented before.

Our first results indicate that segmentation using the FCM algorithm when tumors are present provides the ability to segment normal gray and white matter without including 
tumor as part of either. The commonly used segmentation routines in SPM2 suffer this problem as they rely on an underlying model of the brain which does not include any abnormalities. All of the brains with tumor were segmented more accurately, as compared to the 3D-SPGR with good gray-white matter differentiation, using FCM than with SPM2. The FCM always separated the tumors as different tissue types than either gray, white, or CSF or even the scalp, skull or meninges. For the normal brains, SPM2 was the better choice for segmentation as the FCM consistently underestimated the amount of gray matter, especially in the medial regions of the brain. This may be because the $\mathrm{T}_{2}$ and FLAIR images used displayed some brightening in the middle caused by the dielectric effect seen at 3T which SPM2 seemed to correct in the SPGR data used for segmentation in SPM2. FCM and SPM2 performed more comparably for the estimation of white matter and CSF. Larger voxels, $2 \mathrm{~mm}$ x $2 \mathrm{~mm}$ x $2 \mathrm{~mm}$ were also tried to increase FCM processing speed but this decreased the accuracy of the separation into classes when the FCM results were compared to the SPM2 results.

It is to be noted that comparison of FCM segmentation tissues (GM, WM, and CSF) with SPM2 segmented tissues provides only a method for comparison and not a measurement of error because histological data confirming brain tissue types is not available from live humans. It is possible that future experiments could include histological staining and slicing of an animal brain could be used for such as absolute measurement of tissue type accuracy. The FCM to SPM2 segmentation comparison seems to improve with well registered data sets but since that is the best case for SPM2, this doesn't mean that SPM2 is inherently superior. In the case of tumor subjects the GM, WM, CSF tissues are not separated well by SPM2 or FCM. This may be because the effects of radiation and chemotherapy may change the characteristics of normal tissues.

Our work is an extension of previous studies of tissue segmentation using the fuzzy cmeans algorithm on MRI images that showed the promise of this method ${ }^{39}$. In that study, segmented tissues from FCM were compared to histology and postmortem reports. The segmentation was applied to a single slice of the brain through the tumor with $T_{1}, T_{2}$, Proton density images as feature vectors. However, results indicated that this attempt to 
classify the tumor cells infiltrating into normal tissues was unsuccessful although the edema was successfully segmented from the surrounding tissue. No work was done to determine if the grey matter, white matter, and CSF classes were corrected identified and sample error from the biopsy was present in the results.

In other work, FCM segmentation was compared to various other methods. One method compared the fuzzy and k-nearest neighbor algorithms ${ }^{32}$. For both of these algorithms knowledge-based techniques were used to improve the segmentation after the clustering. Both methods were applied to whole volume MR data sets using the same three mostly anatomical image types as in the other study. They used either the fuzzy or k-nearest neighbor (kNN) algorithm along with regions described by templates to label and classify the tissues after clustering. However this method was unable to categorize the tissues with abnormalities since a prior model of normal brains cannot be used in this case.

In another study ${ }^{45}$, a semi-supervised FCM (SFCM) algorithm was applied to tumor subjects and the results were compared to kNN, gray level thresholding and seed growing. Seed growing methods require manual selection of voxels of interest; such methods can be highly operator dependent. Using their SFCM algorithm tumor volumes were measured and the values did not consistently match the values from the other techniques as measured by the inter- and intra-operator coefficients of variation. Moreover, the SFCM was performed on semi-supervised and masked (skull and meninges stripped) images so the advantage of automation was reduced. In this study the problem of a gold standard was also mentioned. For human brain tumor segmentation only immediately post-mortem scans can be validated without sampling error. Presurgical or pre-biopsy scans can be helpful especially if performed under image guidance.

The unsupervised segmentation of MR images using different algorithms has also been compared in earlier studies ${ }^{28}$. The algorithms used in this study were two types of fuzzy c-means: one which uses a predefined number of classes and another based on maximumentropy which does not. The results demonstrated that each of these methods can successfully segment normal brain MR images but they did not attempt to apply either 
algorithm to patient data.

An understanding of the fuzzy clustering algorithm is required before applying the algorithm to MR data sets. Important parameters like the number of clusters (tissues), m (degree of fuzziness), and threshold values of the FCM algorithm need to be considered. This technique, when used with a smaller number of classes, resulted in a segmentation that merged the tissue types. Moreover as the number of feature vectors or number of clusters increases, the FCM algorithm calculation times increase linearly. The time consumed for segmenting approximately 100 slices is about 90 minutes. An important future step for this project is the minimization of processing time by translating the MATLAB code into $\mathrm{C}$ and reevaluating the algorithm. That is a large project that could take a significant amount of time.

SPM2 is a commonly and popularly used software package to segment brain images. SPM2 is based on the maximum-likelihood mixture model clustering algorithm and can be performed using just $\mathrm{T}_{1}$-weighted or with multimodal inputs. In the description of the SPM technique ${ }^{27}$, the segmentation results were verified only by comparison to the input $\mathrm{T}_{1}$-wtd., or $\mathrm{T}_{2}$-wtd. images. In order for the tissue segmentation to work well using SPM2, good contrast MRI images are a requirement. If the images are not registered well, the normalization and hence segmentation of the images using SPM2 is poor as seen in Figure A-20. The segmentation accuracy with respect to misregistration of images has been found to follow a normal distribution using SPM2 ${ }^{27}$. Moreover, when voxels contain partial volume effects, they are often segmented incorrectly as gray matter particularly. Since segmentation is done using a priori probability images, segmenting of abnormal tissues will almost always yield poor results. This is a primary limitation of SPM2 software is for the segmenting tissues when abnormalities are present. 
For this project, the MRI data sets did not require co-registration because of patient movement or image distortion, the images were co-registered and normalized only in order to compare them to the segmentation using SPM2 software. The additional advantage of interpolation to a higher spatial resolution also improved the FCM results. For some patients, identical slice prescriptions were not possible across the MRI modalities, image reslicing allowed us to work around this problem.

In spite of the intuitive sense that adding diffusion-weighted images would improve the tissue segmentation, our results indicate that adding DWI to the feature vectors did not yield better results for these images as seen in Figure 4.15 and Table 6, Table 7. This may be due to the fact that diffusion weighted images may contain distortions.

Another confound was that the dielectric effect generated-inhomogeneities present in the more inferior portion of the MRI data sets added a spurious class to the data as seen in Figure A-1. This effect caused FCM results for the upper half of the slices to differ from the whole brain segmentations. The more superior slices which do not demonstrate this effect show good separation of tissues as seen from Figure A-4. In the future the FCM segmentation should be applied to images acquired on a 1.5T scanner to test if this effect can be removed. Alternately, intensity smoothing filters could be applied or phased-array surface head coils could be used at 3T.

In summary and conclusion, the FCM technique developed for this thesis work can be used as for tumor segmentation and in detecting brain tumor region changes along with MRI images. The method is better than SPM2 for selecting normal gray and white matter in brains that have tumors although not as good as SPM2 for segmentation of normal brains. Future work should include an exploration of the application of this technique to 1.5T images, application to other types of abnormalities, accuracy determination through animal model work, and improvements to speed of processing. 


\section{Bibliography}

1. Moseley M. Personal Communication.

2. Dowsett DJ, Kenny PA, Johnston ER. The physics of diagnostic imaging. London, New York: Chapman \& Hall Medical; 1998.

3. NessAiver M. All You Really Need to Know about MRI Physics: Simply Physics; 1996.

4. Bushong SC. Magnetic Resonance Imaging: Physical and Biological Principles: C.V. Mosby; 1996.

5. Nishimura D. Principles of Magnetic Resonance Imaging: Course notes; 1994.

6. Kuperman V. Magnetic Resonance Imaging - Physical Principles and Applications. NewYork: Academic Press; 2000.

7. Institute NC. What you need to know about brain tumors.

8. Kleihues P, Webster K, Cavanee WK. World Health Organization Classification of Tumours: Pathology and Genetics: Tumours of the Nervous System: Who Publications Centre.; 2000.

9. Vaupel P, Kallinowski F, Okunieff P. Blood flow, oxygen and nutrient supply, and metabolic microenvironment of human tumors: a review. Cancer Res 1989;49(23):6449-6465.

10. Goldmann E. The growth of malignant disease in man and the lower animals, with special reference to the vascular system. Proc Royal Acad Med 1907(1):1-13.

11. Gray LH, Conger AD, Ebert M, Hornsey S, Scott OC. The concentration of oxygen dissolved in tissues at the time of irradiation as a factor in radiotherapy. Br J Radiol 1953;26(312):638-648.

12. Pomper MG, Port JD. New techniques in MR imaging of brain tumors. Magn Reson Imaging Clin N Am 2000;8(4):691-713.

13. Gray L, MacFall J. Overview of diffusion imaging. Magn Reson Imaging Clin N Am 1998;6(1):125-138.

14. Holodny AI, Ollenschlager M. Diffusion imaging in brain tumors. Neuroimaging Clin N Am 2002;12(1):107-124.

15. Stejskal EO, Tanner JE. Spin Diffusion Measurements: Spin Echoes in the Presence 
of a Time-Dependent Field Gradient. J Chem Phys 1965;42:288-292.

16. Sugahara T, Korogi Y, Kochi M, et al. Usefulness of diffusion-weighted MRI with echo-planar technique in the evaluation of cellularity in gliomas. J Magn Reson Imaging 1999;9(1):53-60.

17. Kassner A, Buckley DL, Jackson A. Assessment of angiogenesis in enhancing cerebral tumors using contrast-enhanced MRI. Medica Mundi 2002;46(1):39-43.

18. Villringer A, Rosen BR, Belliveau JW, et al. Dynamic imaging with lanthanide chelates in normal brain: contrast due to magnetic susceptibility effects. Magn Reson Med 1988;6(2):164-174.

19. Thompson HK, Jr., Starmer CF, Whalen RE, McIntosh HD. Indicator Transit Time Considered as a Gamma Variate. Circ Res 1964;14:502-515.

20. Aksoy FG, Lev MH. Dynamic contrast-enhanced brain perfusion imaging: technique and clinical applications. Semin Ultrasound CT MR 2000;21(6):462-477.

21. Schaefer PW, Lev MH, Buchbinder BR, González RG. Clinical Applications of Functional MRI. In Radiology: Diagnosis, Imaging, Intervention. Philadelphia: Lippincott; 1997.

22. Aronen HJ, Gazit IE, Louis DN, et al. Cerebral blood volume maps of gliomas: comparison with tumor grade and histologic findings. Radiology 1994;191(1):41-51.

23. McKnight TR. Proton magnetic resonance spectroscopic evaluation of brain tumor metabolism. Semin Oncol 2004;31(5):605-617.

24. Bruhn H, Frahm J, Gyngell ML, et al. Noninvasive differentiation of tumors with use of localized H-1 MR spectroscopy in vivo: initial experience in patients with cerebral tumors. Radiology 1989;172(2):541-548.

25. Alger JR. Brain Cancer and Magnetic Resonance Spectroscopy: Academic Press; 2002.

26. Bensaid AM, Hall LO, Clarke LP, Velthuizen RP. MRI Segmentation Using Supervised and Unsupervised Methods. 1991 Oct. 31st-Nov. 3rd; Orlando, Florida.

27. Ashburner J, Friston KJ. Voxel-based morphometry-The methods. Neuroimage 2000;11(6):805-821.

28. Bezdek JC, Hall LO, Clarke LP. Review of MR image segmentation techniques using pattern recognition. Med Phys 1993;20(4):1033-1048. 
29. Brandt ME, Bohan TP, Kramer LA, Fletcher JM. Estimation of CSF, white and gray matter volumes in hydrocephalic children using fuzzy clustering of MR images. Comput Med Imaging Graph 1994;18(1):25-34.

30. Hall LO, Bensaid AM, Clarke LP, Velthuizen RP, Silbiger ML, Bezdek JC. A Comparison of Neural Network and Fuzzy Clustering Techniques in Segmenting Magnetic Resonance Images of the Brain. IEEE Transactions on Neural Networks 1992;3(5).

31. Bezdek JC. Pattern Recognition with Fuzzy Objective Function Algorithms. New York: Kluwer Academic Publishers; 1981.

32. Clark M, Hall L, Goldgof Dea. MRI segmentation using fuzzy clustering techniques: Integrating knowledge. IEEE Engineering in Medicine and Biology 1994;13(5):730742.

33. Masulli F, Artuso M, Bogu's P, Schenone. Fuzzy Clustering Methods for the Segmentation of Multivariate Medical Images. In: Mares M MR, Novak V, Ramik J, Stupnanova A,, editor; 1997. p 123-128.

34. Vannier M, Speidel C, Rickmans D. Magnetic resonance imaging multispectral tissue classification. News Physiol Sci 1988;3:148-154.

35. Vannier MW, Butterfield RL, Jordan D, Murphy WA, Levitt RG, Gado M. Multispectral analysis of magnetic resonance images. Radiology 1985;154(1):221224.

36. Höppner F, Klawonn F, Kruse R, Runkler T. Fuzzy Cluster Analysis: Wiley; 1999. $289 \mathrm{p}$.

37. Klawonn F, Höppner F. What is Fuzzy About Fuzzy Clustering?-Understanding and Improving the Concept of the Fuzzifier. In: M.R. Berthold HJL, E. Bradley, R. Kruse, C. Borgelt, ed. Advances in Intelligent Data Analysis. Berlin: V. Springer, 2003; 254264.

38. Toolbox M. Mathworks Inc.

39. Phillips WE, 2nd, Velthuizen RP, Phuphanich S, Hall LO, Clarke LP, Silbiger ML. Application of fuzzy c-means segmentation technique for tissue differentiation in MR images of a hemorrhagic glioblastoma multiforme. Magn Reson Imaging 1995;13(2):277-290. 
40. Cox RW. AFNI: software for analysis and visualization of functional magnetic resonance neuroimages. Comput Biomed Res 1996;29(3):162-173.

41. Talairach J, Tournoux P. Co-planar stereotaxic atlas of the human brain. Thieme Medical 1988.

42. Friston KJ, Ashburner J, Frith CD, Poline JB, Heather JD, Frackowiak RSJ. Spatial registration and normalization of images. Human Brain Mapping 1995;3:165-189.

43. Ashburner J. Computational Neuroanatomy [PhD thesis]: University College London; 2000.

44. Dale AI. Bayes or Laplace? An examination of the origin and early applications of Bayes' theorem. Arch Hist Exact Sci 1982;27(1):23-47.

45. Vaidyanathan M, Clarke LP, Hall LO, et al. Monitoring brain tumor response to therapy using MRI segmentation. Magn Reson Imaging 1997;15(3):323-334. 


\section{Appendix A}


Normal subject (N1): Inputs to FCM (FLAIR, T2, SPGR, SPGRGD)
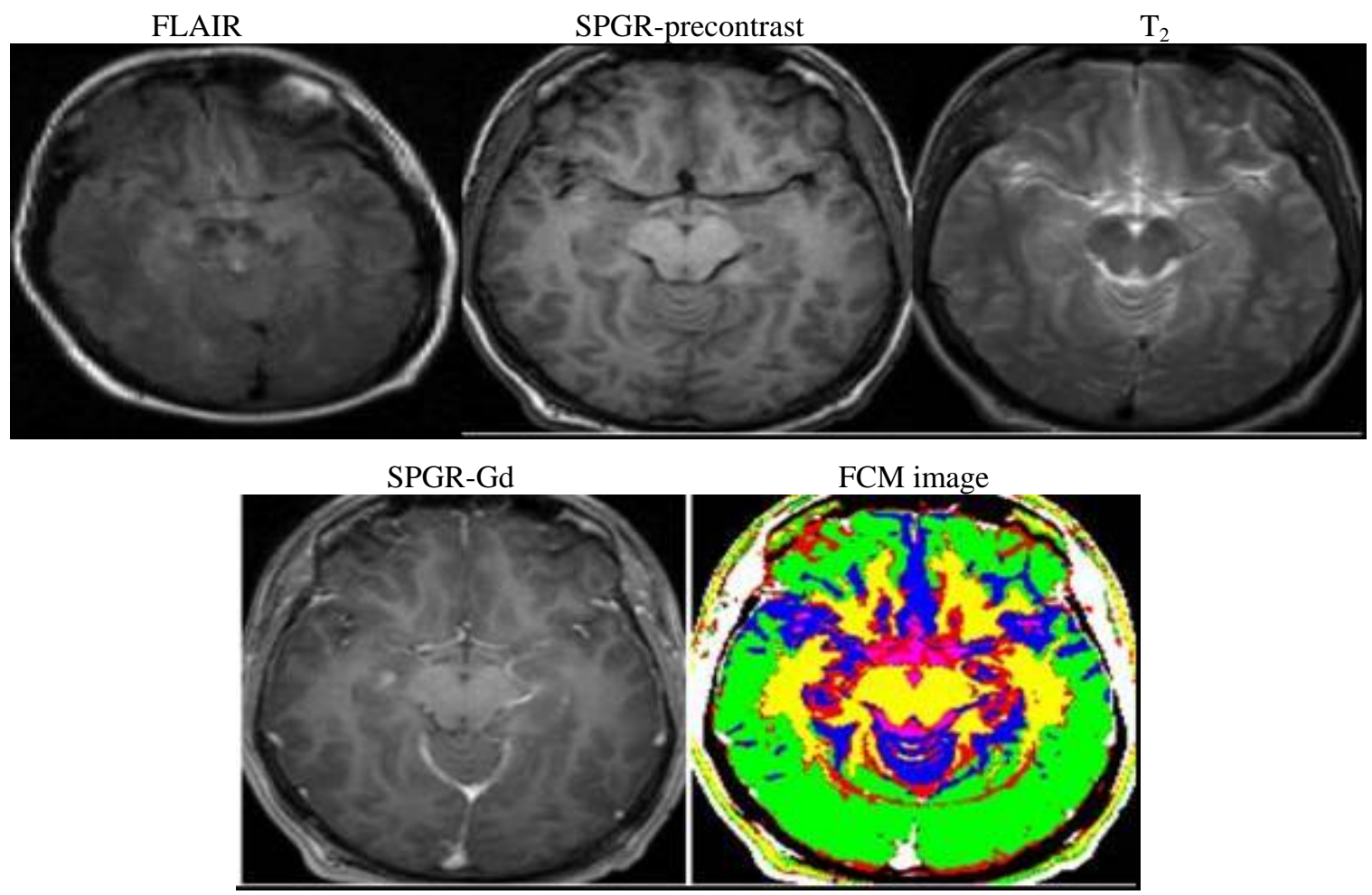

Figure A-1: MRI volume data sets segmented into six classes (tissue) types.
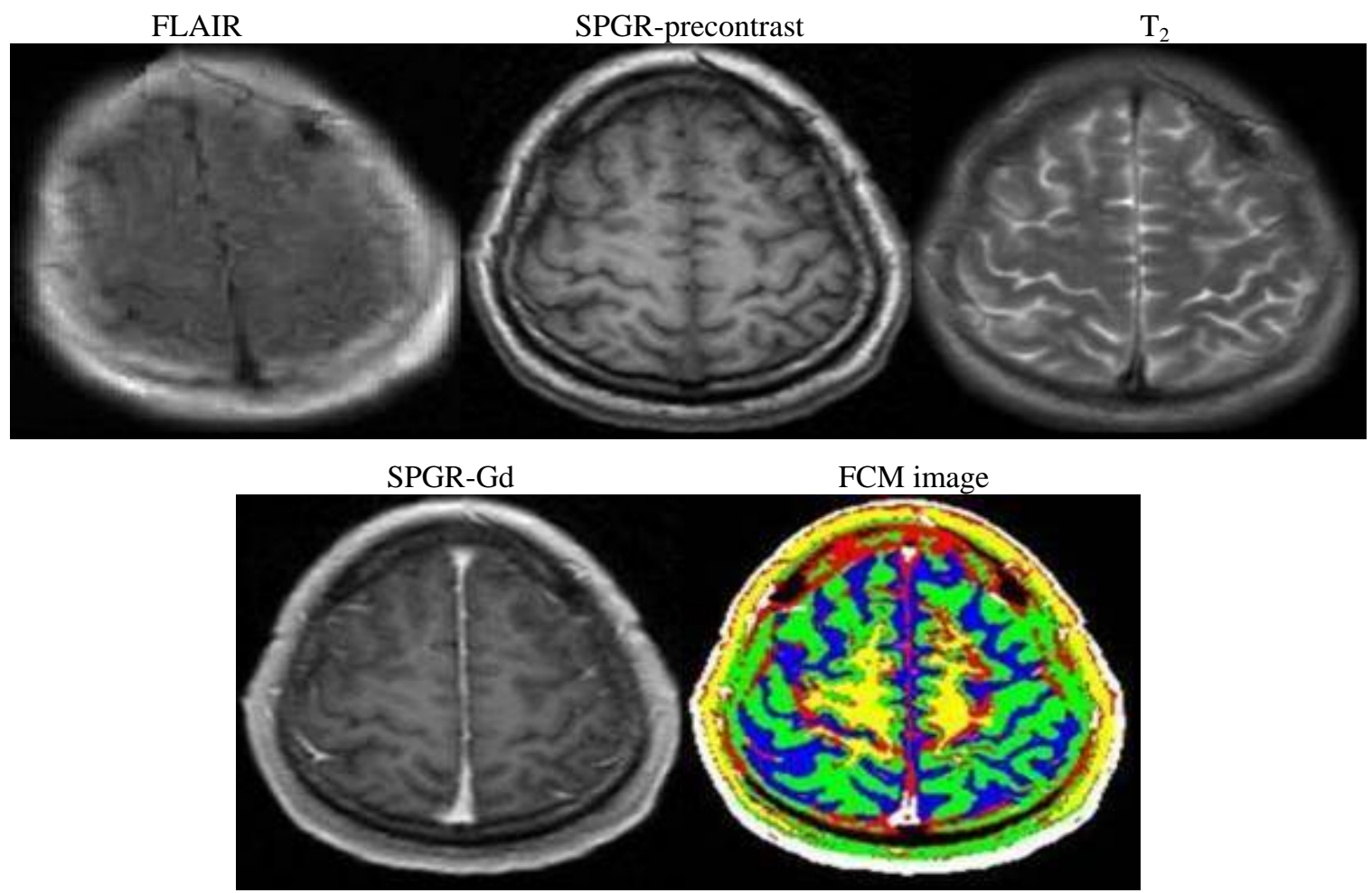

Figure A-2: MRI volume data sets at another slice location segmented into six classes (tissue) types. 
Normal subject (N2): Inputs to FCM (FLAIR, T2, SPGR, SPGRGD)
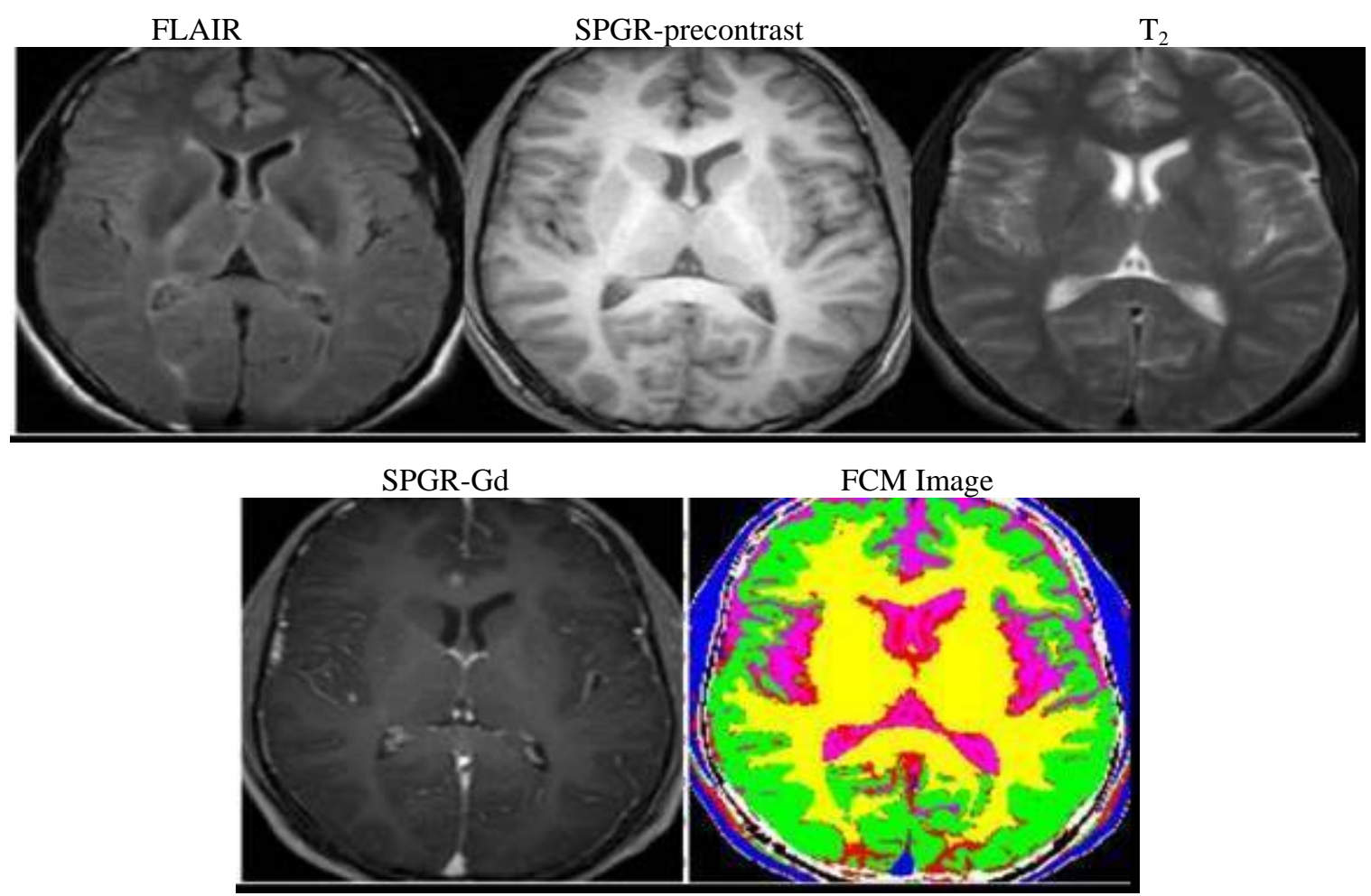

Figure A-3: MRI volume data sets segmented into six classes (tissue) types.
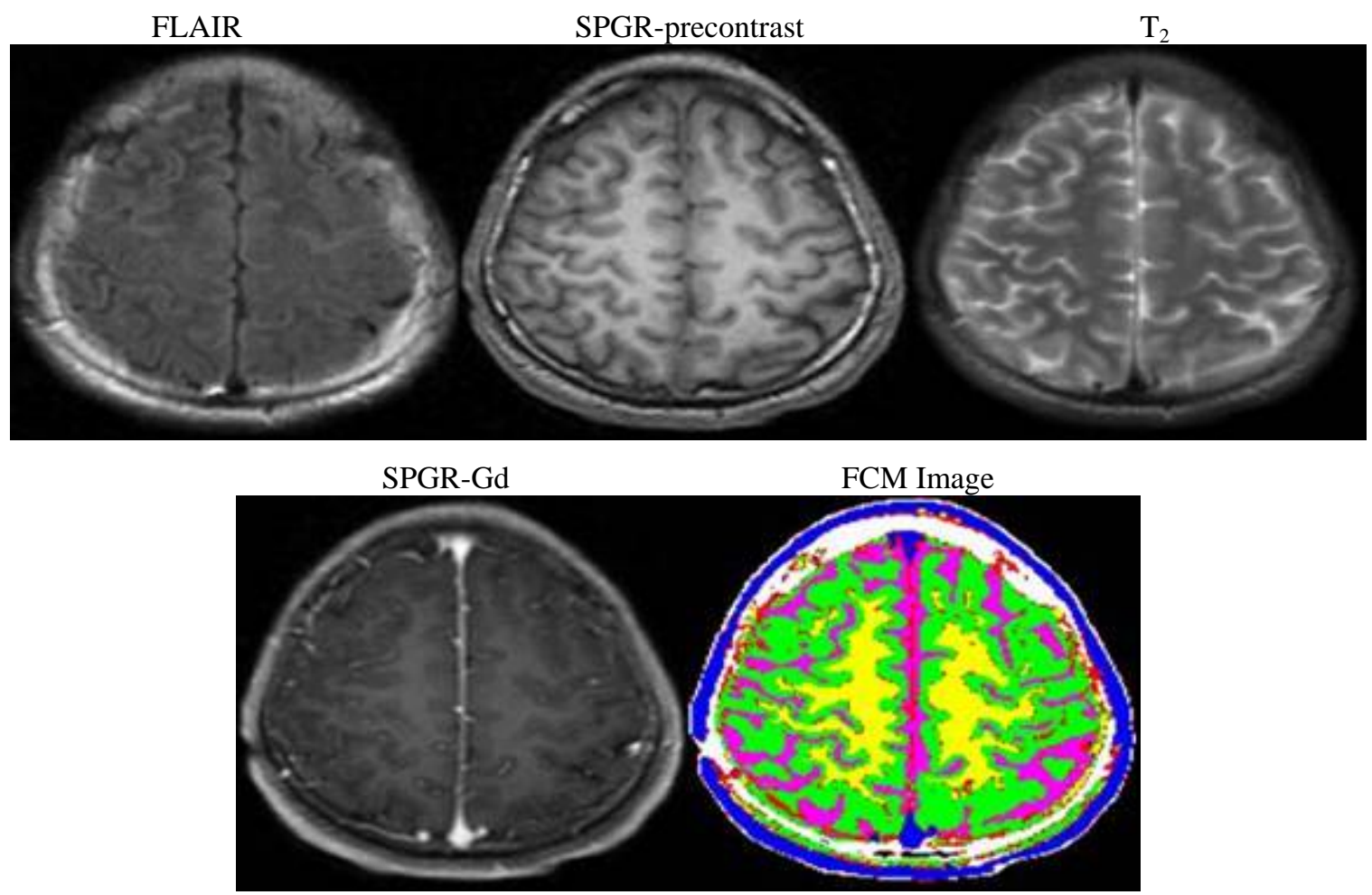

Figure A-4: MRI volume data sets at another slice location segmented into six classes (tissue) types. 
Normal subject (N3): Inputs to FCM (FLAIR, T2, SPGR, SPGRGD)
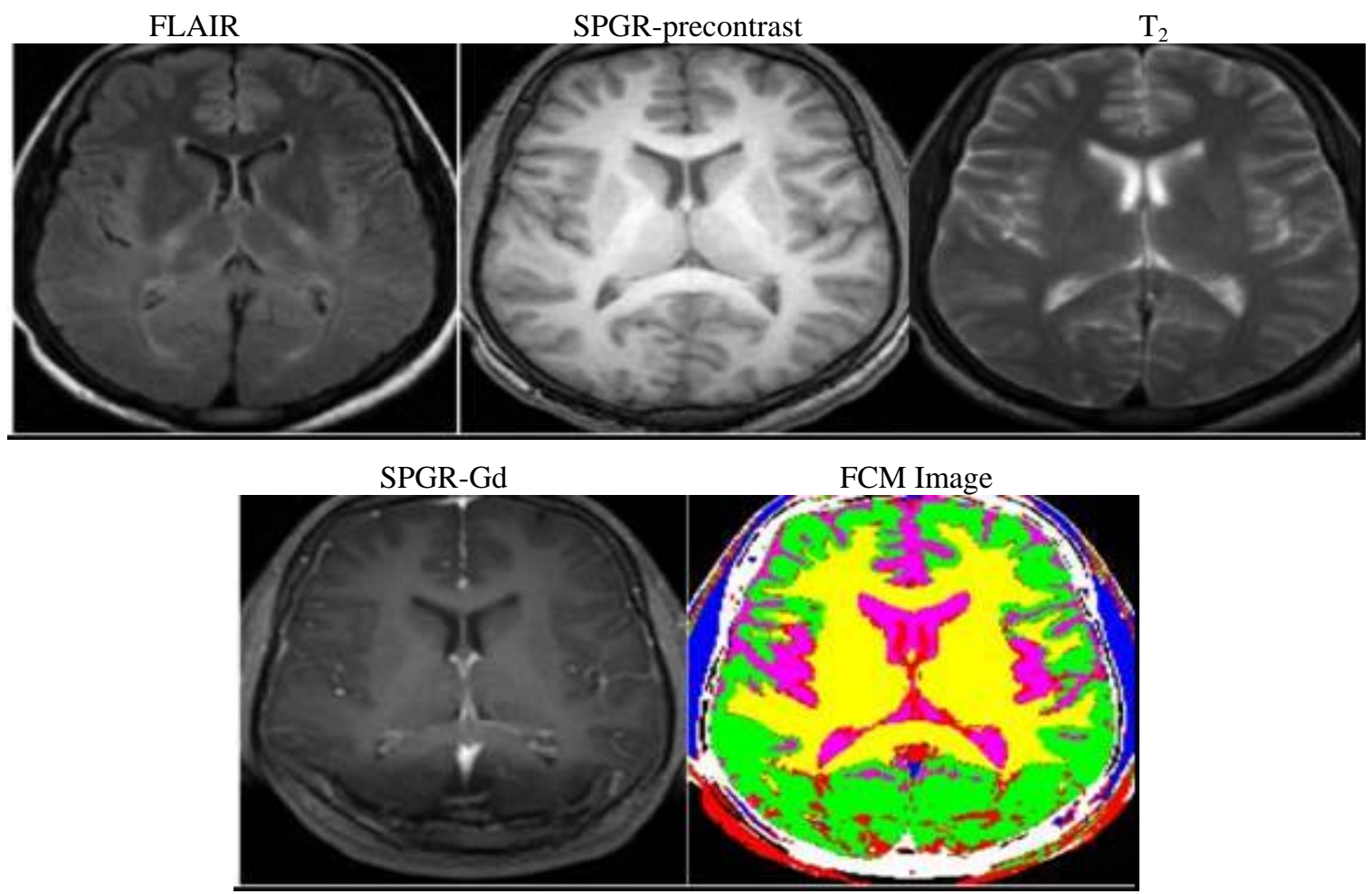

Figure A-5: MRI volume data sets segmented into six classes (tissue) types.
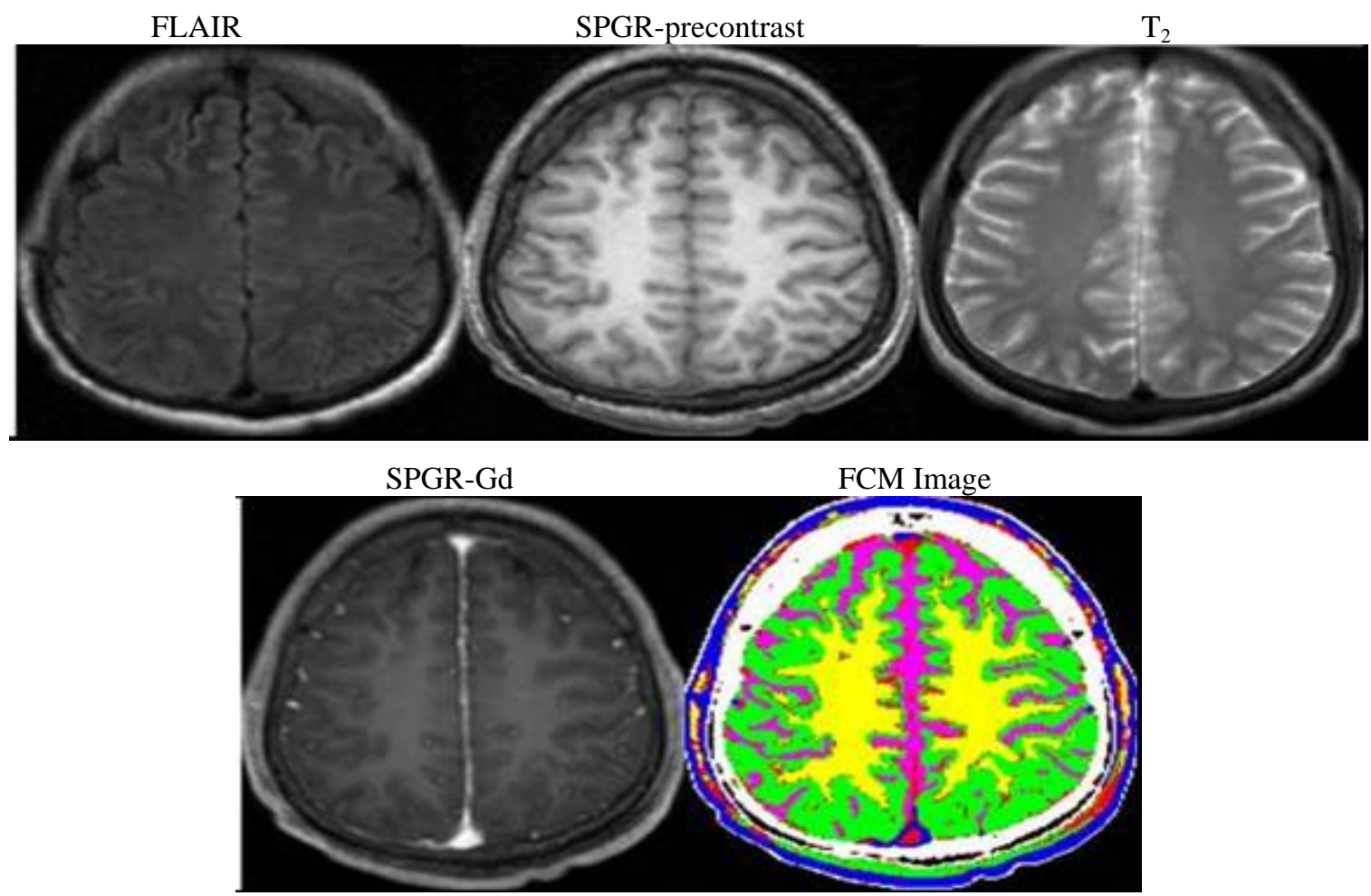

Figure A-6: MRI volume data sets at another slice location segmented into six classes (tissue) types. 
Normal subject (N4): Inputs to FCM (FLAIR, T $_{2}$, SPGR, SPGRGD)
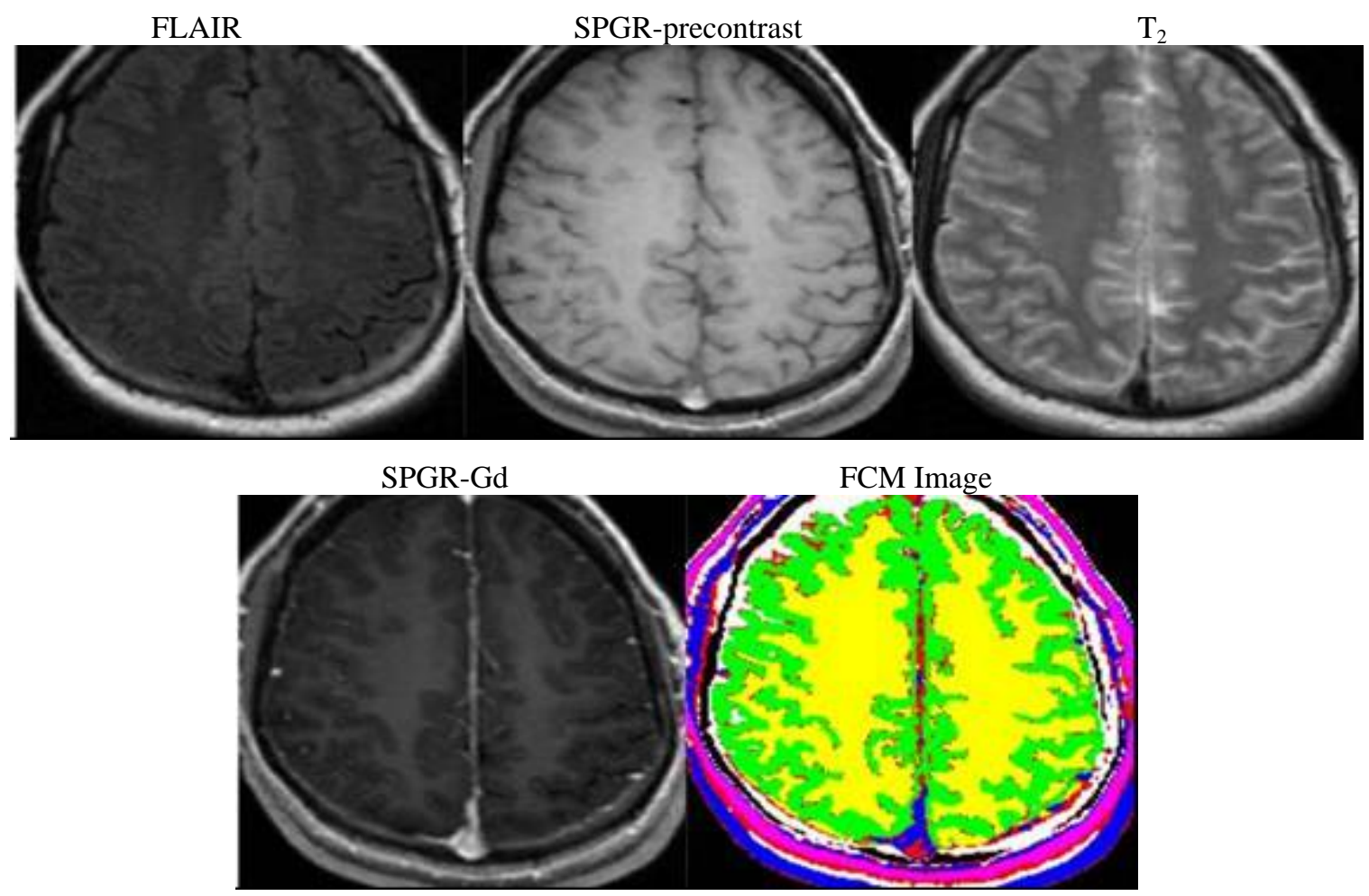

Figure A-7: MRI volume data sets segmented into six classes (tissue) types.
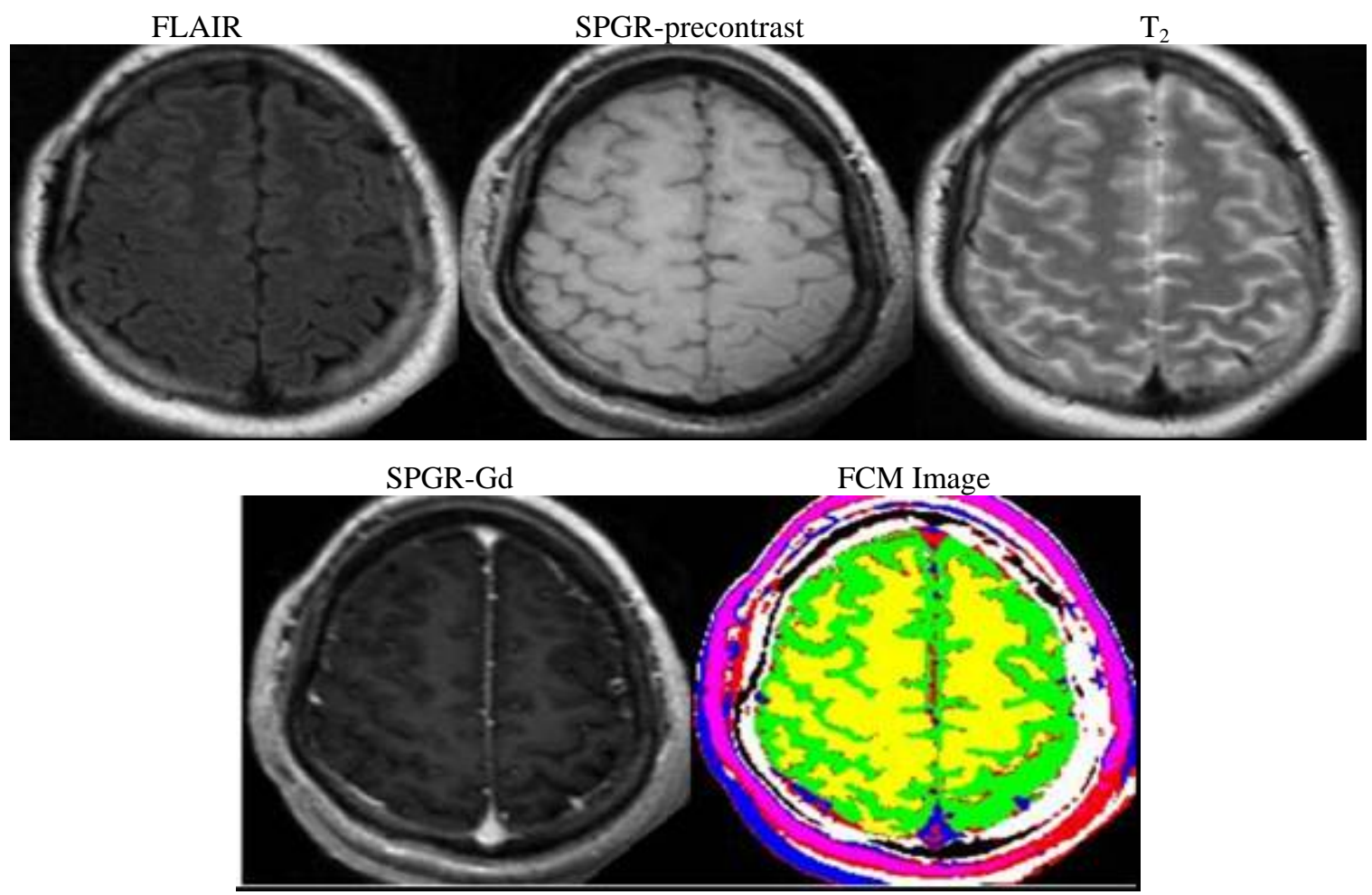

Fig A-8: MRI volume data sets at another slice location segmented into six classes (tissue) types. 
Tumor patient (P1): Inputs to FCM (FLAIR, T2, SPGR, SPGRGD)
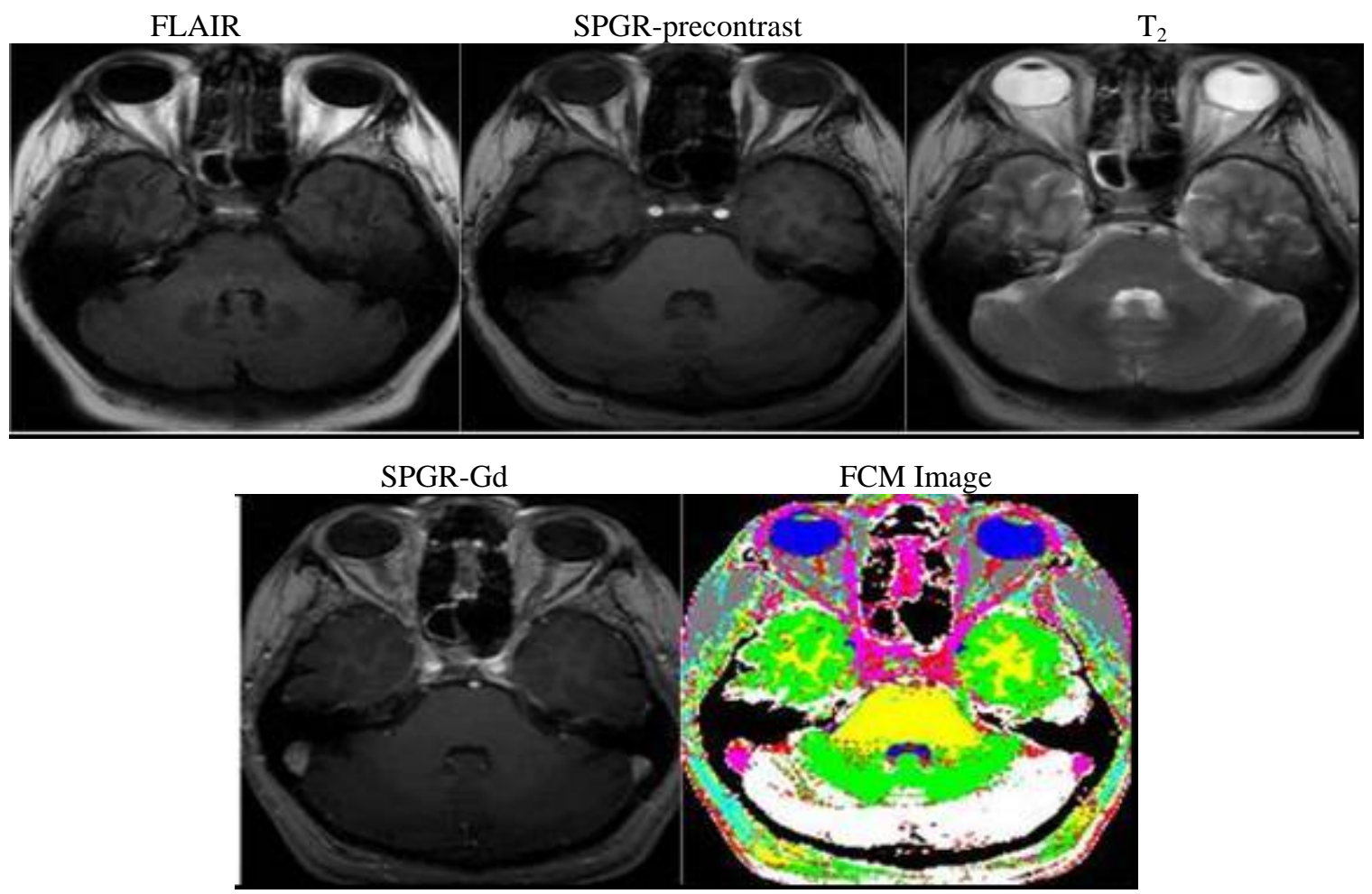

Figure A-9: MRI volume data sets segmented into nine classes (tissue) types.
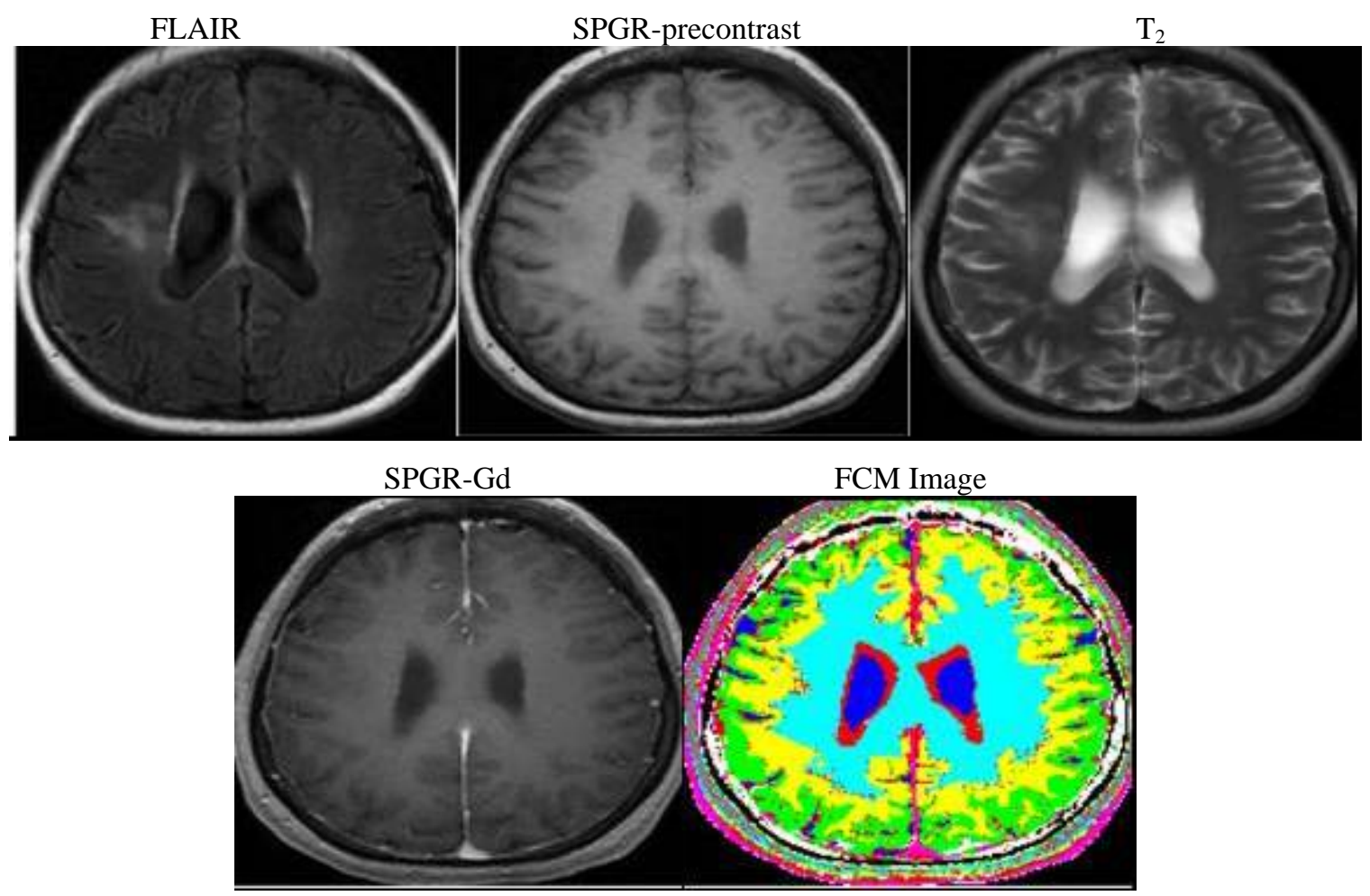

Figure A-10: MRI volume data sets at another slice location segmented into nine classes (tissue) types. 
Tumor patient (P2): Inputs to FCM (FLAIR, T, SPGR, SPGRGD)
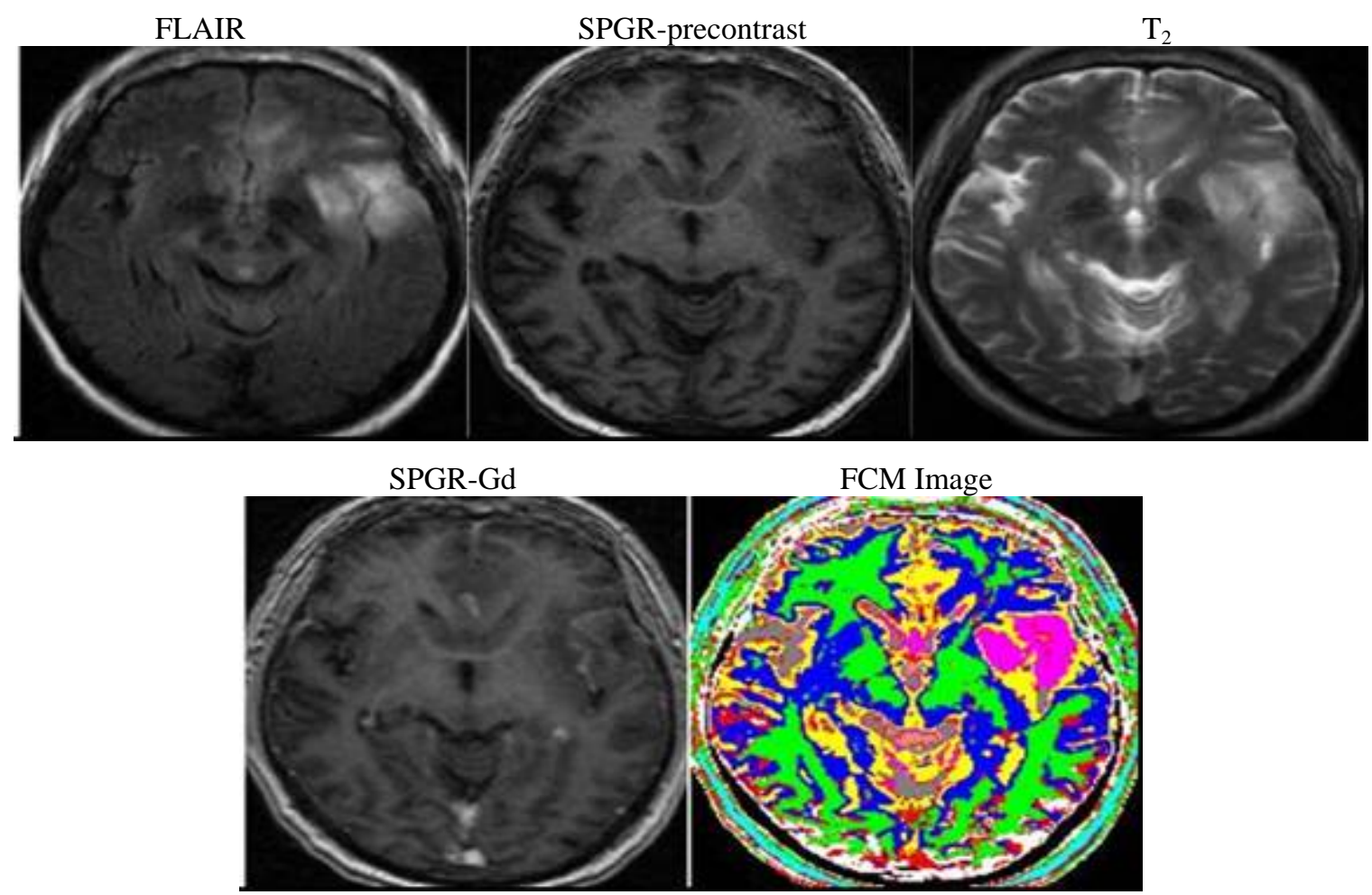

Figure A-11: MRI volume data sets segmented into nine classes (tissue) types.
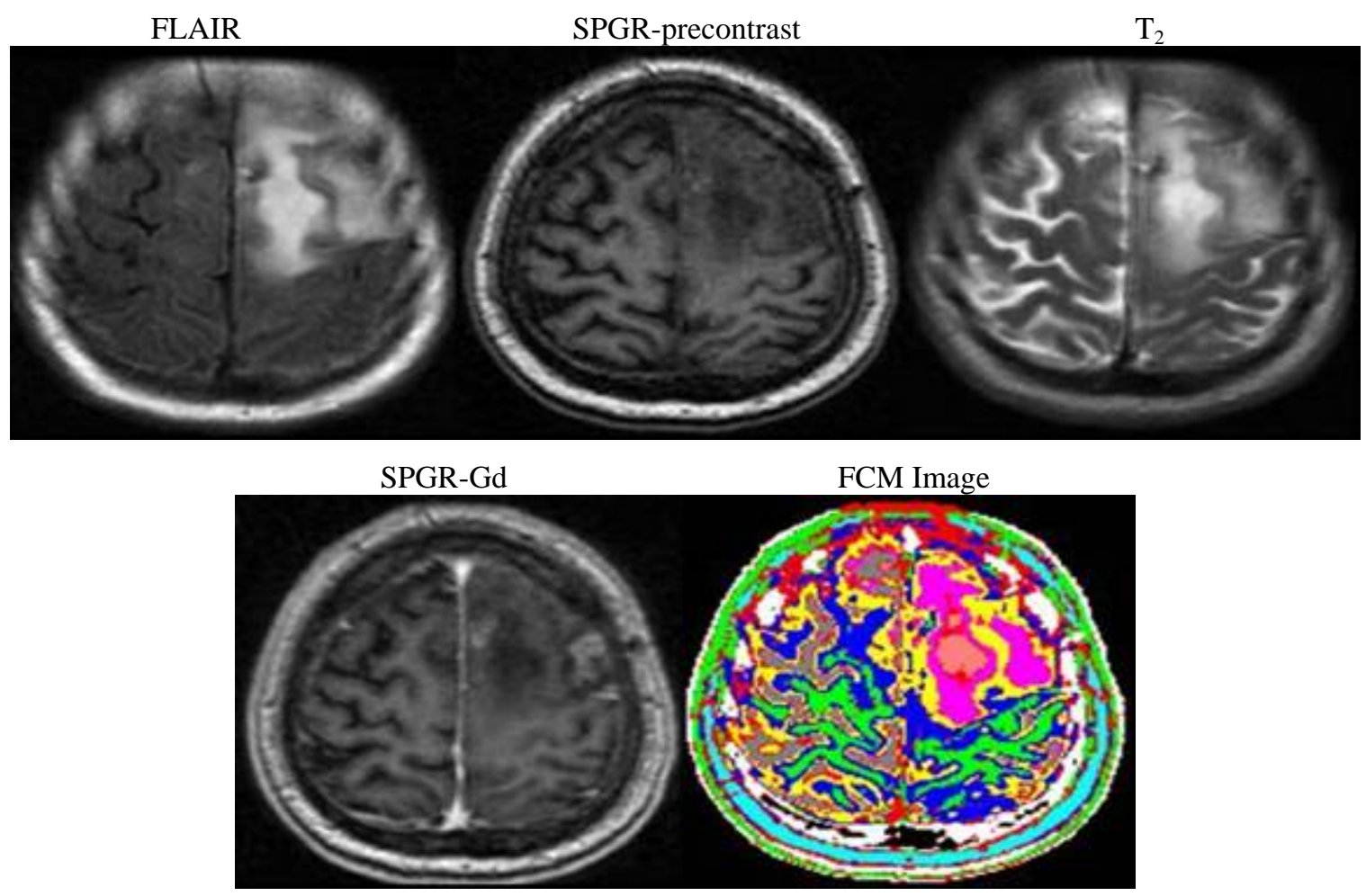

Figure A-12: MRI volume data sets at another slice location segmented into nine classes (tissue) types. 
Tumor patient (P3): Inputs to FCM (FLAIR, T2, SPGR, SPGRGD)
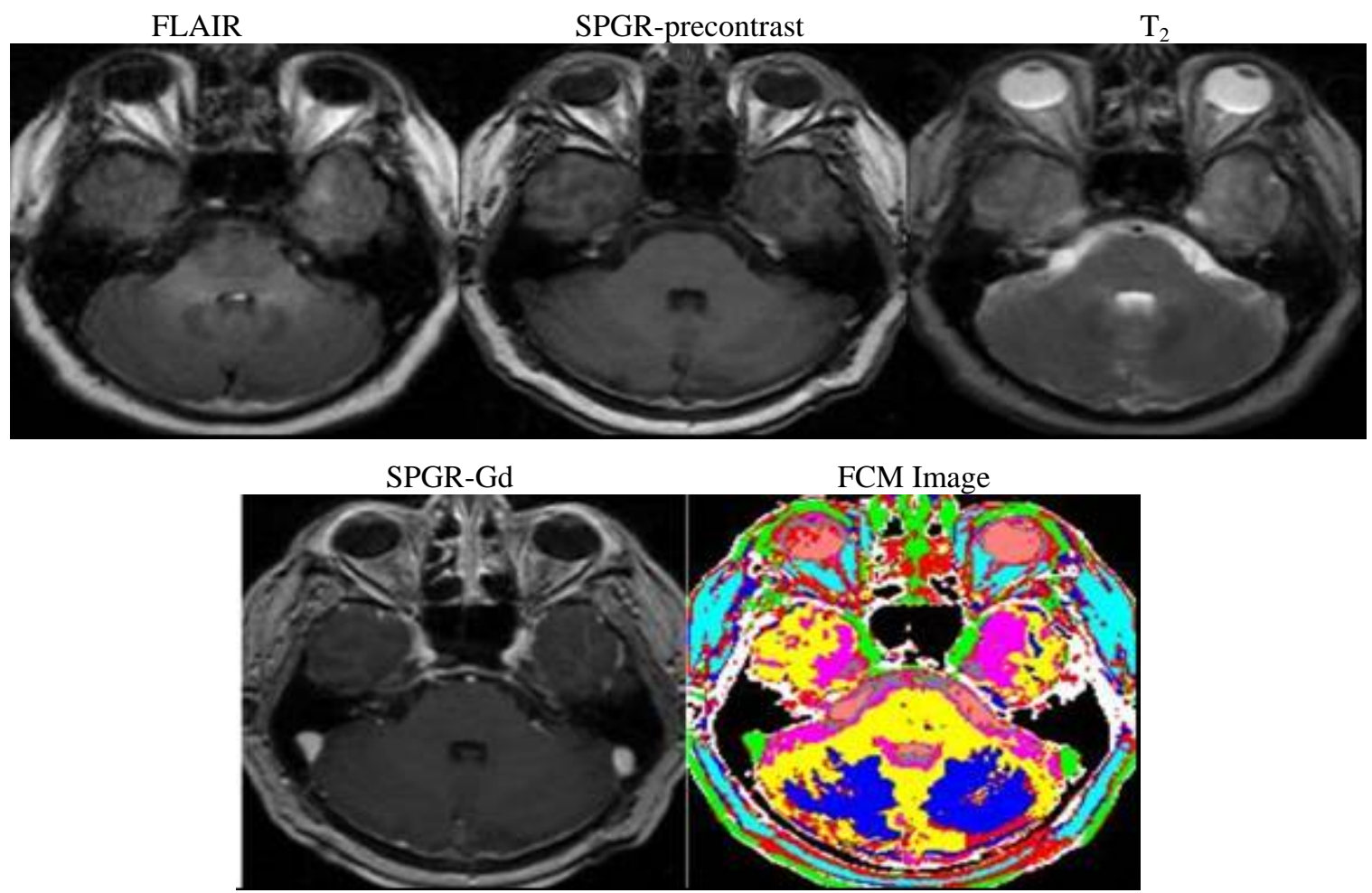

Figure A-13: MRI volume data sets segmented into nine classes (tissue) types.
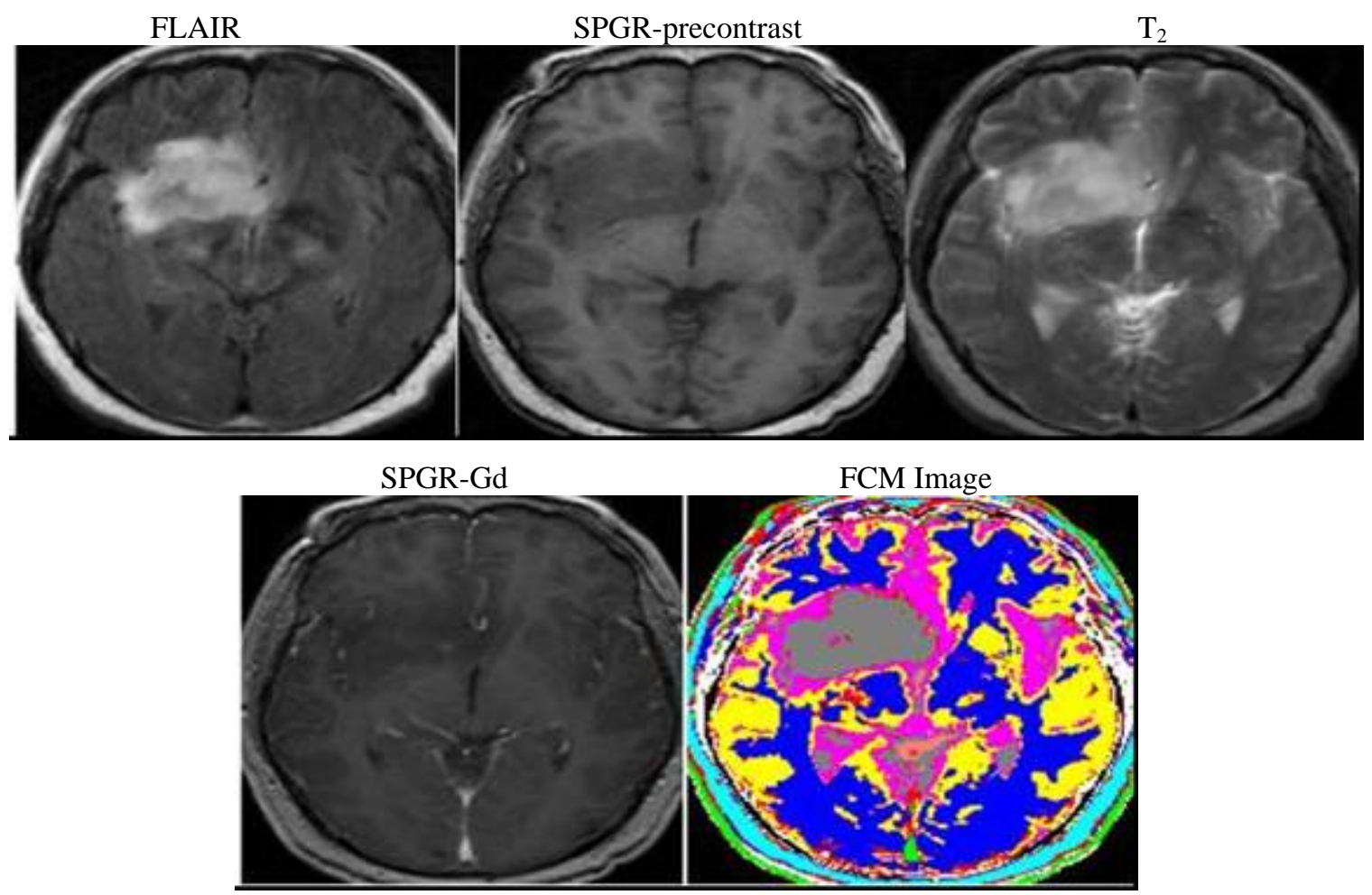

Figure A-14: MRI volume data sets at another slice location segmented into nine classes (tissue) types. 
Tumor patient(P4): Inputs to FCM (FLAIR, T2, SPGR, SPGRGD)
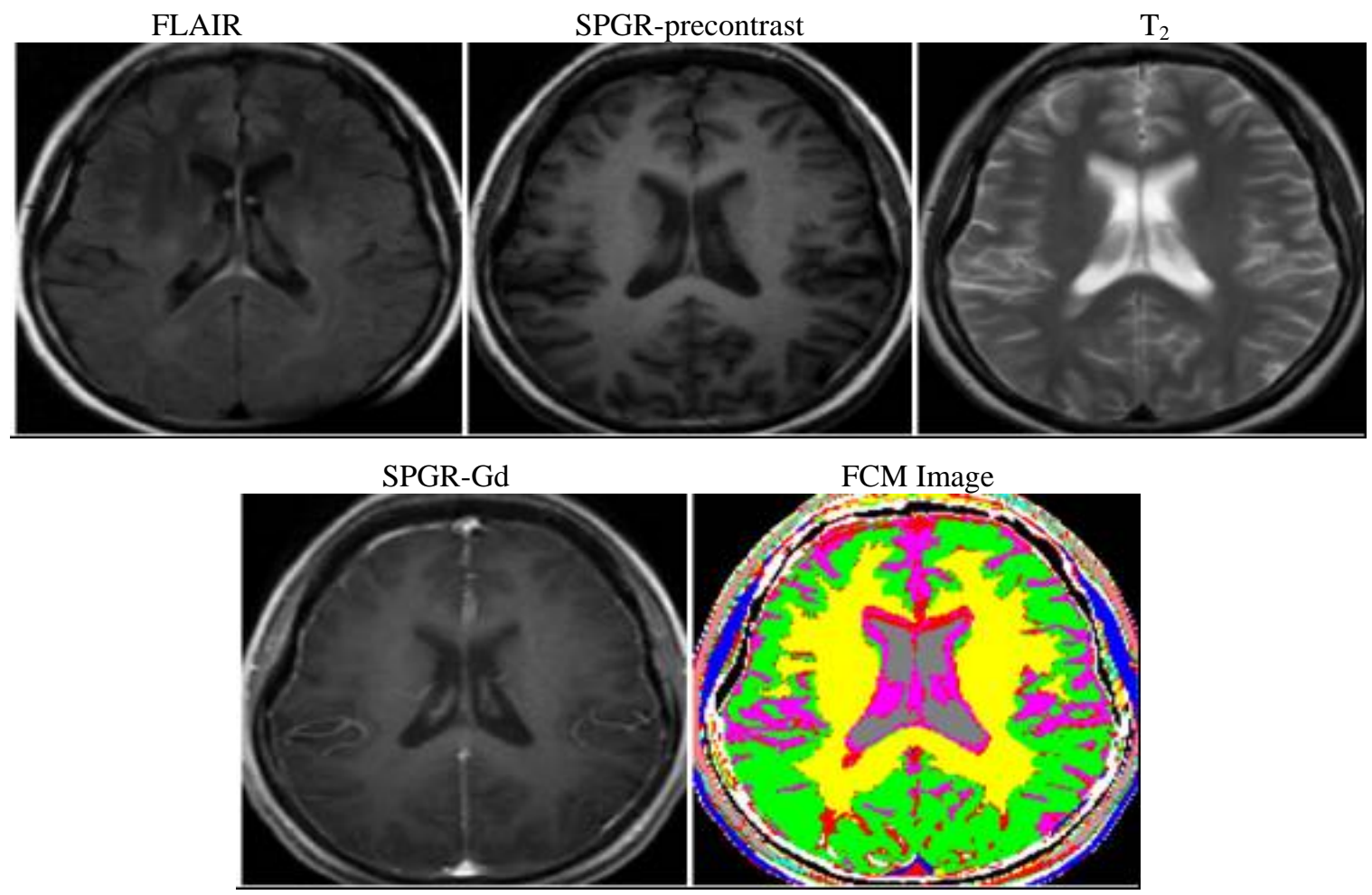

Figure A-15: MRI volume data sets segmented into nine classes (tissue) types.
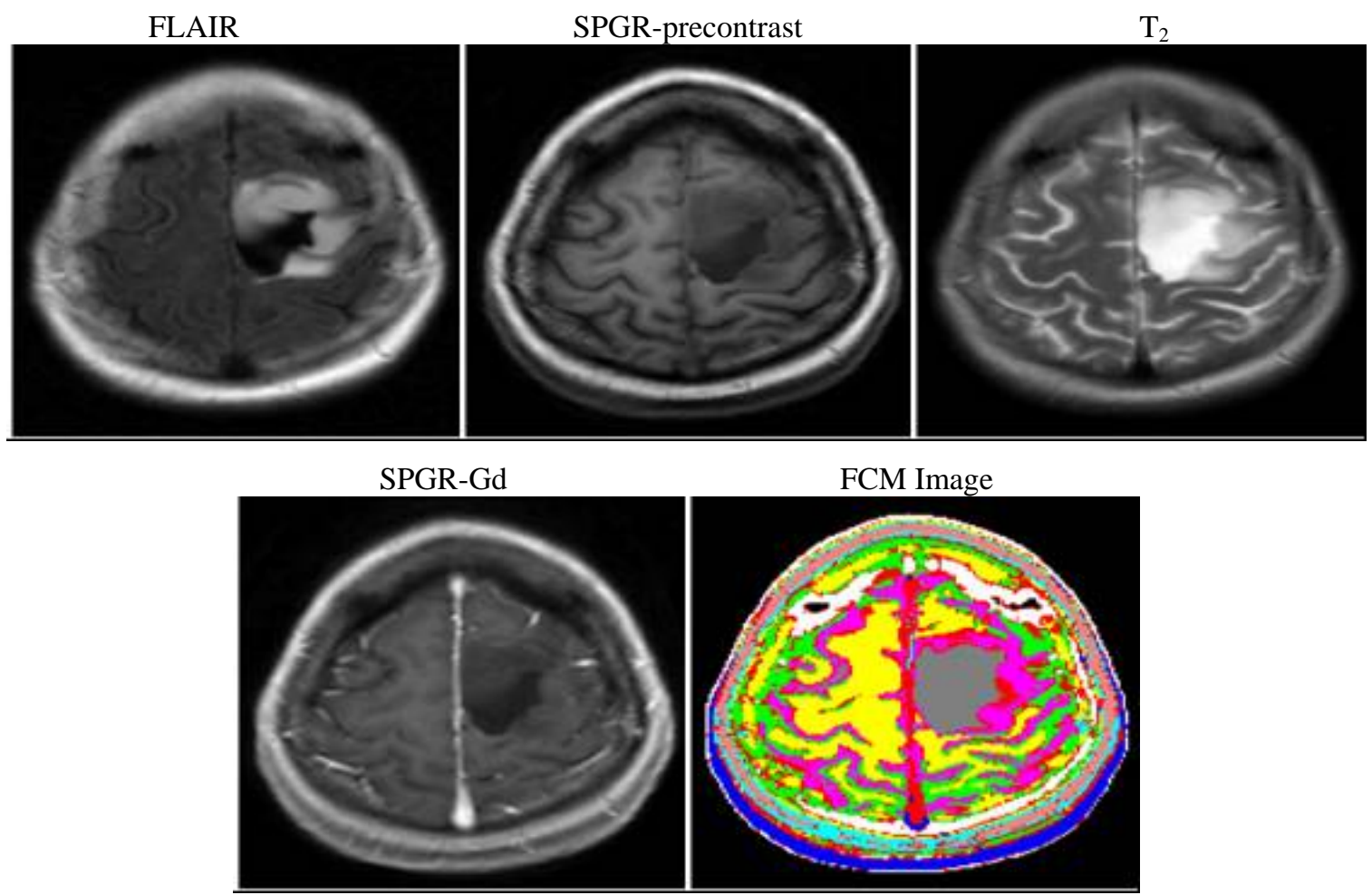

Figure A-16: MRI volume data sets at another slice location segmented into nine classes (tissue) types. 
Normal subject (N1): Comparison between FCM and SPM segmented images.

FCM:

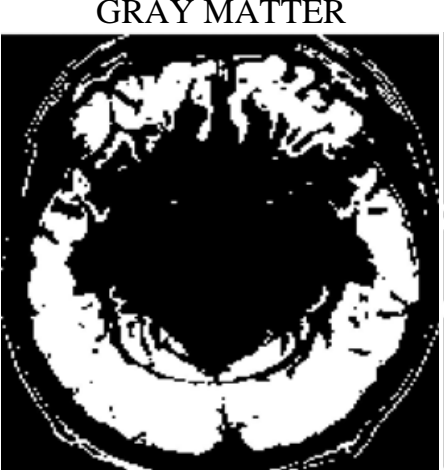

WHITE MATTER

CSF
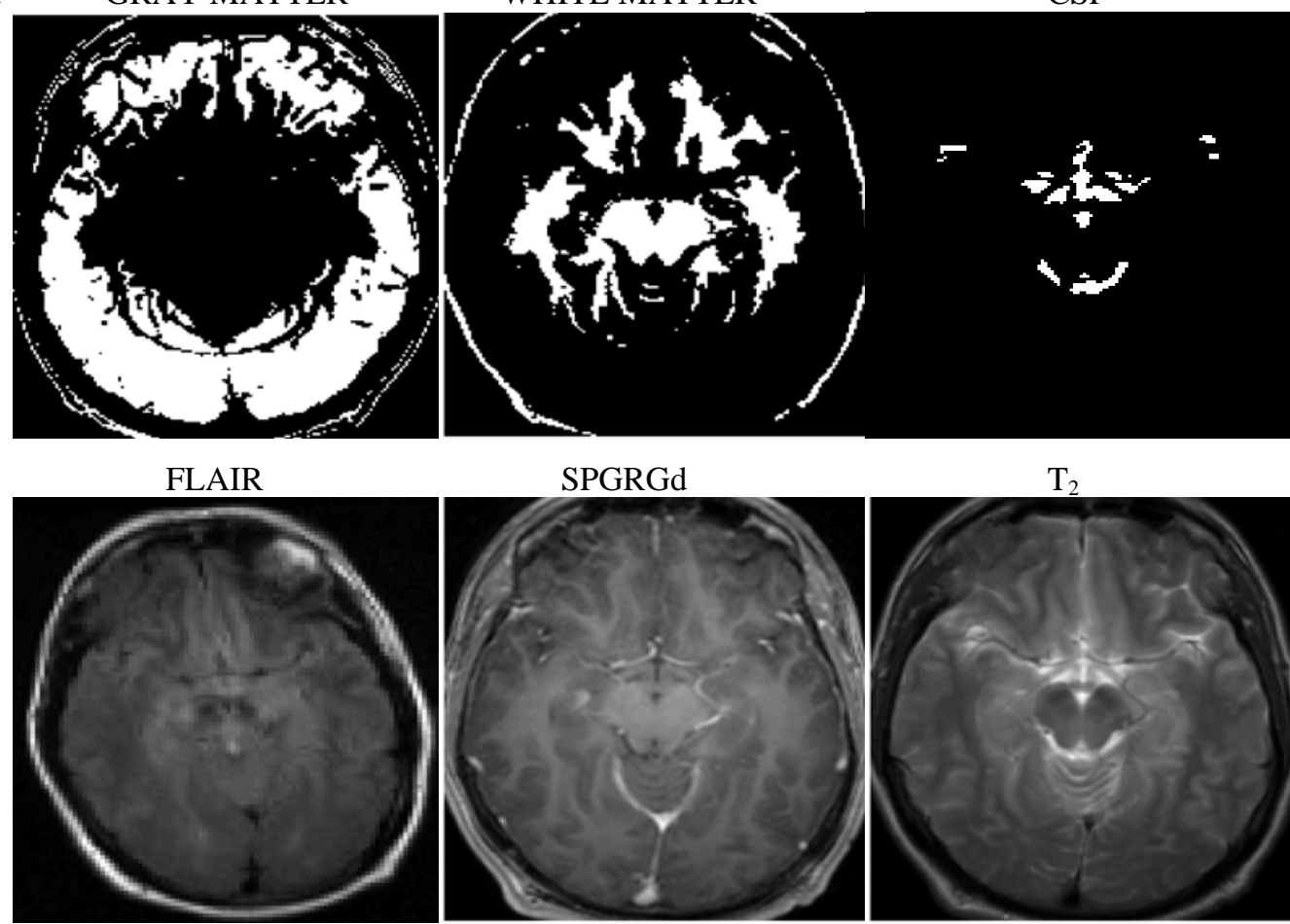

SPGRGd

$\mathrm{T}_{2}$
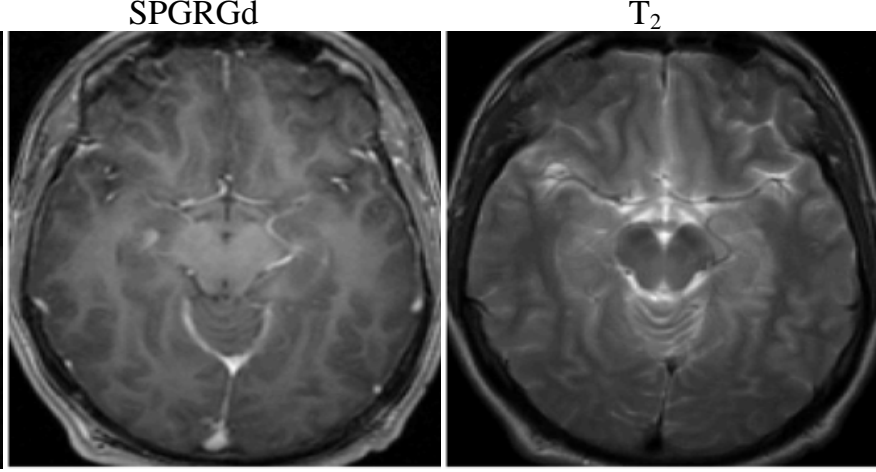

SPM:

GRAY MATTER

WHITE MATTER

CSF
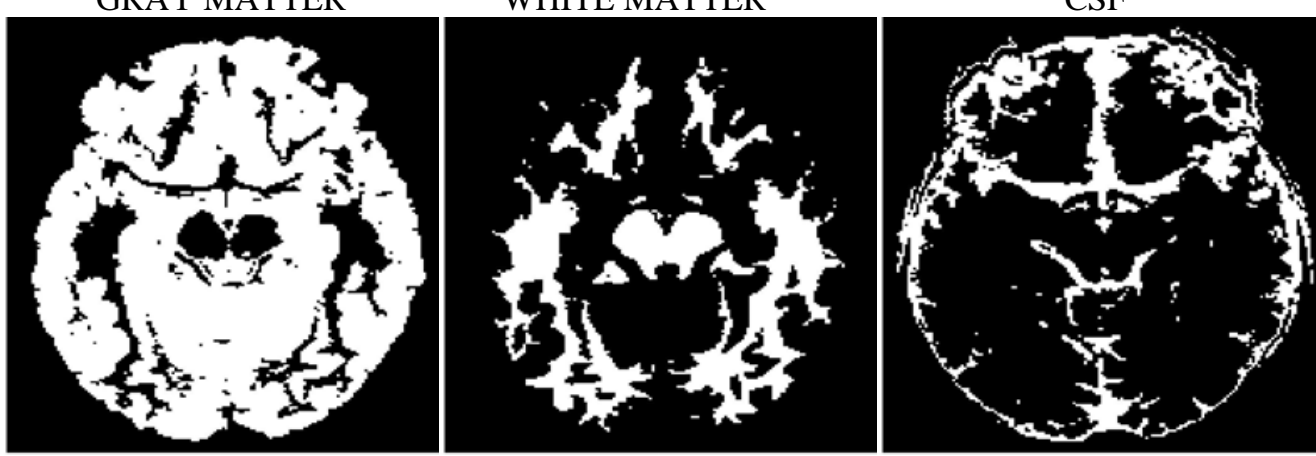

Diff. image: GRAY MATTER

WHITE MATTER

CSF

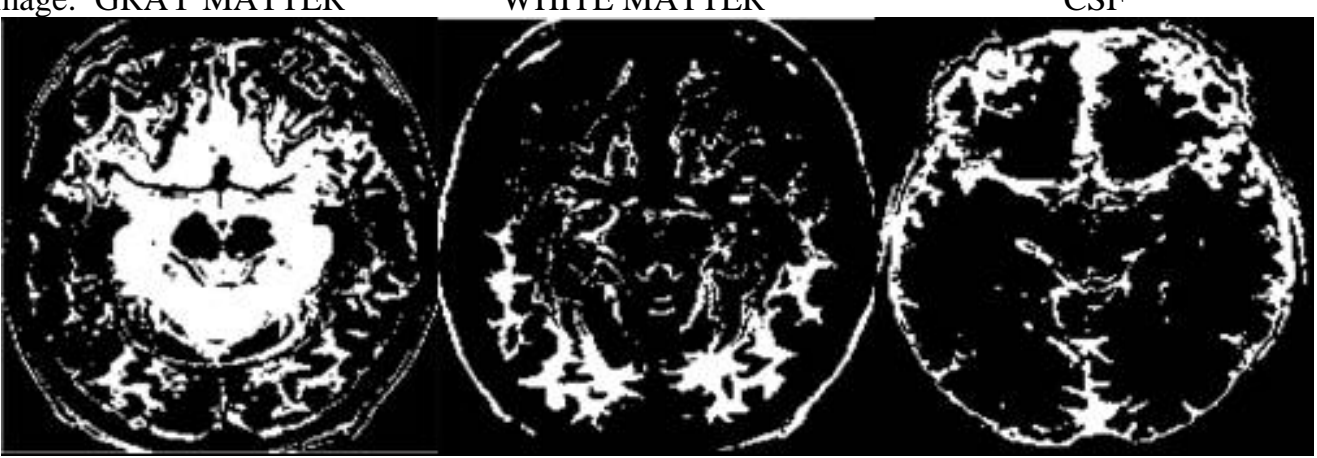

Figure A-17: Comparison of tissues between FCM and SPM segmentation of N1. 
Normal subject (N2): Comparison between FCM and SPM segmented images.

FCM:

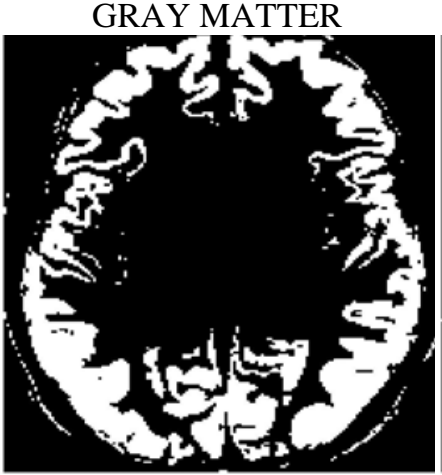

WHITE MATTER
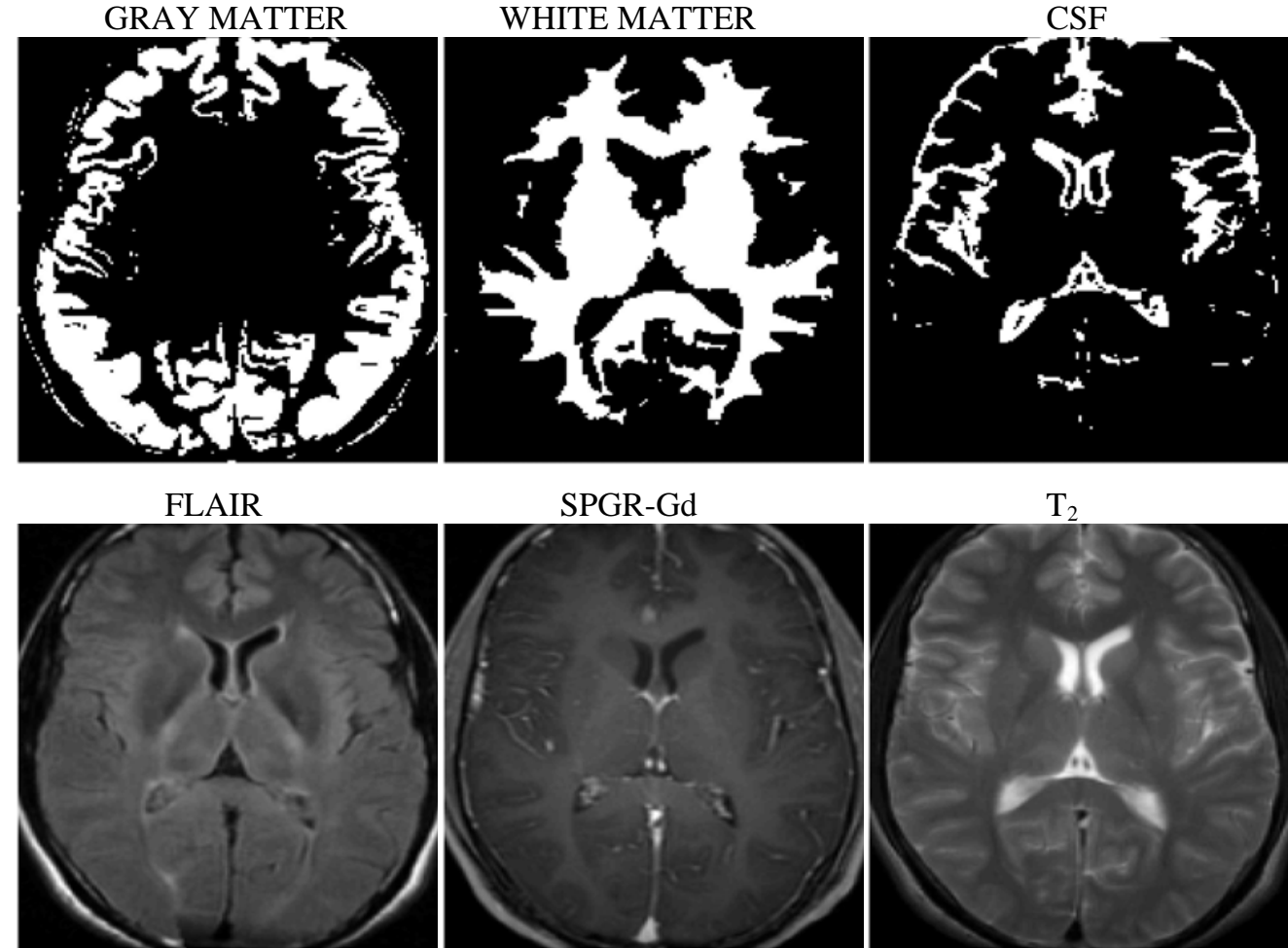

SPGR-Gd

$\mathrm{T}_{2}$
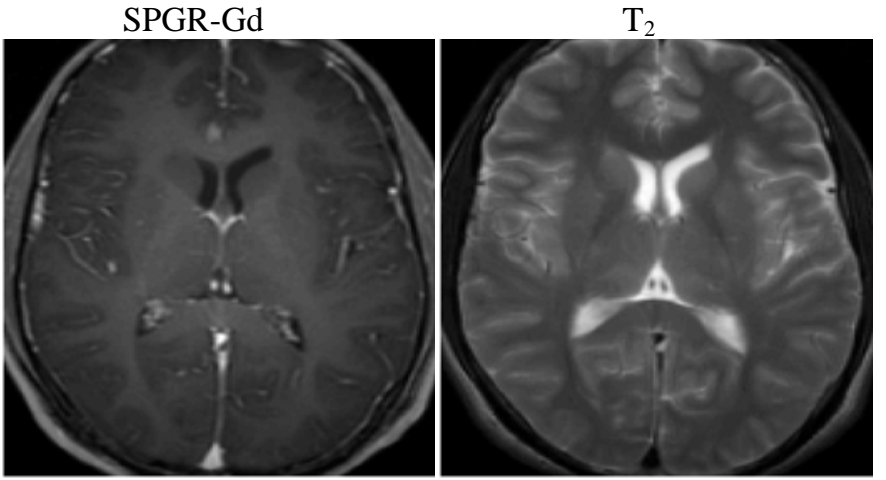

SPM:

GRAY MATTER

WHITE MATTER
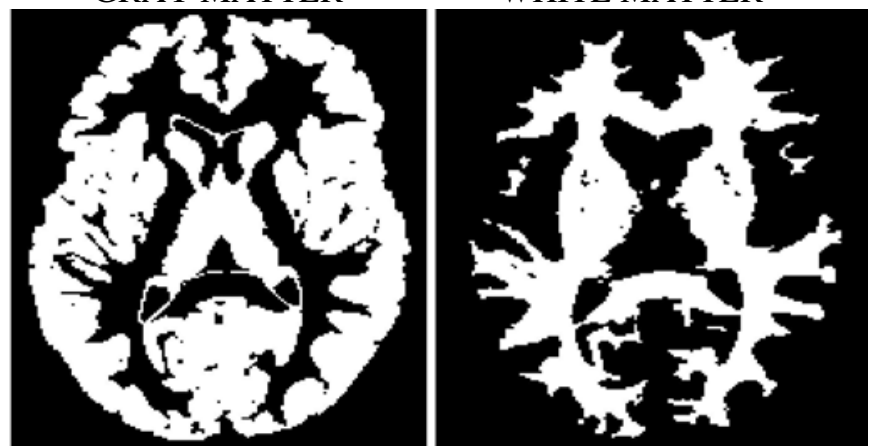

CSF

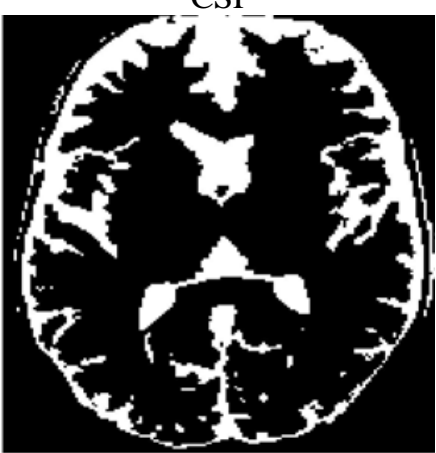

Diff. image: GRAY MATTER

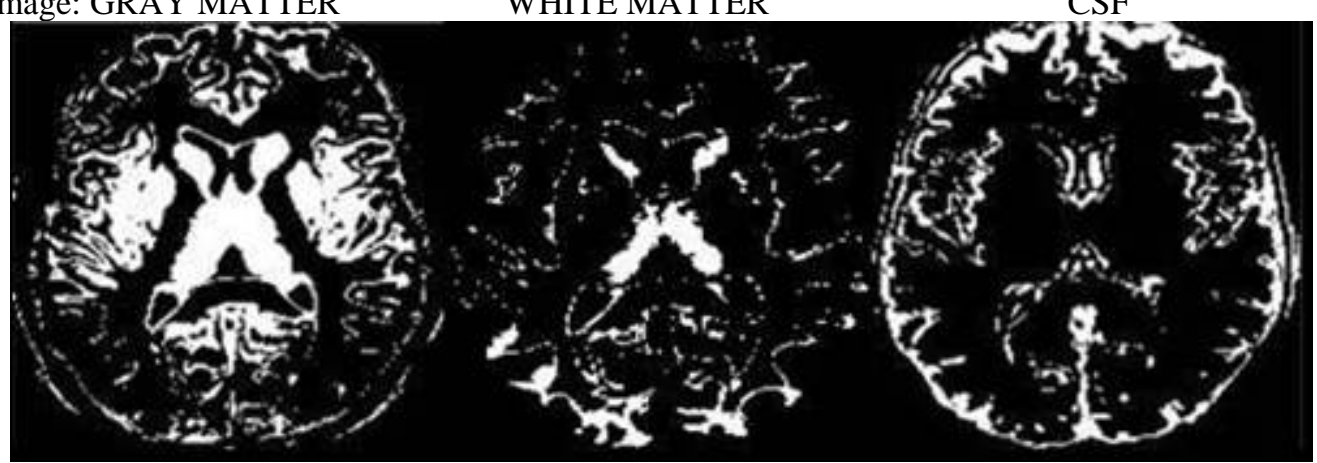

Figure A-18: Comparison of tissues between FCM and SPM segmentation of N2. 
Normal subject (N3): Comparison between FCM and SPM segmented images.

FCM:

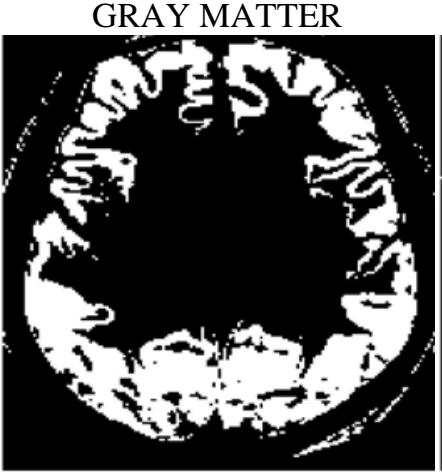

WHITE MATTER
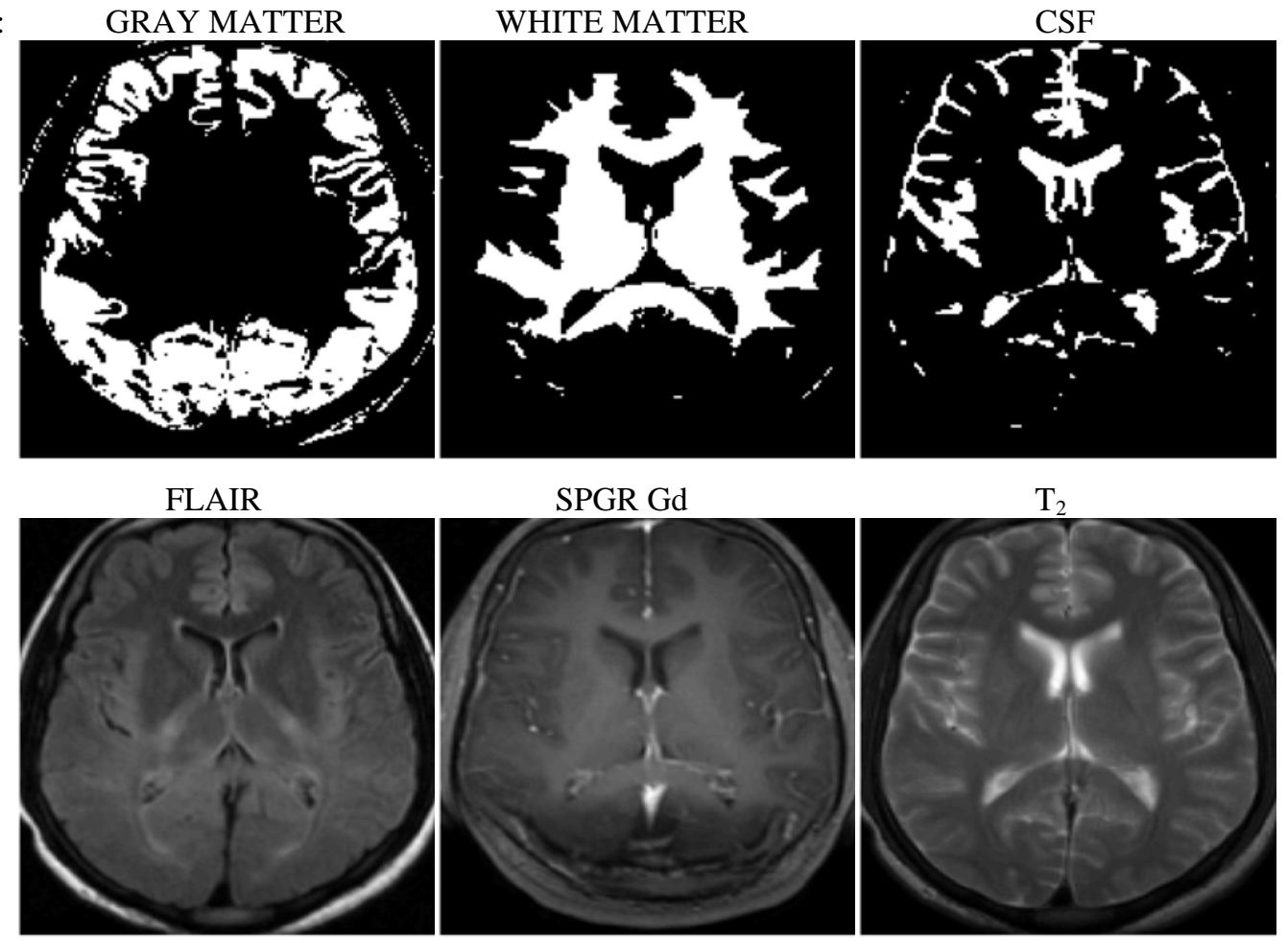

SPM:

GRAY MATTER

WHITE MATTER
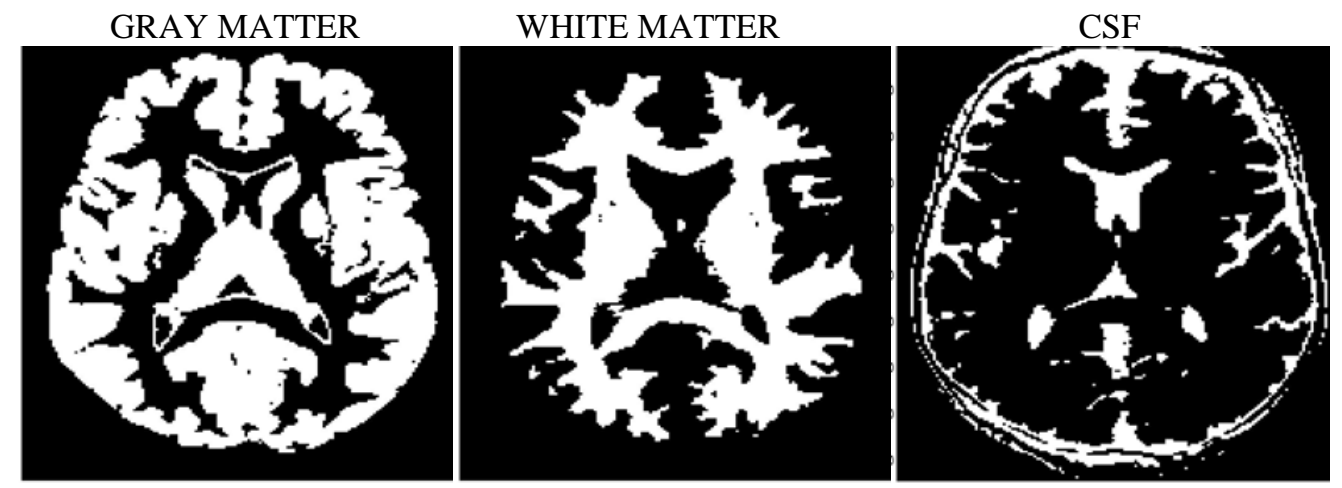

Diff. image: GRAY MATTER

WHITE MATTER

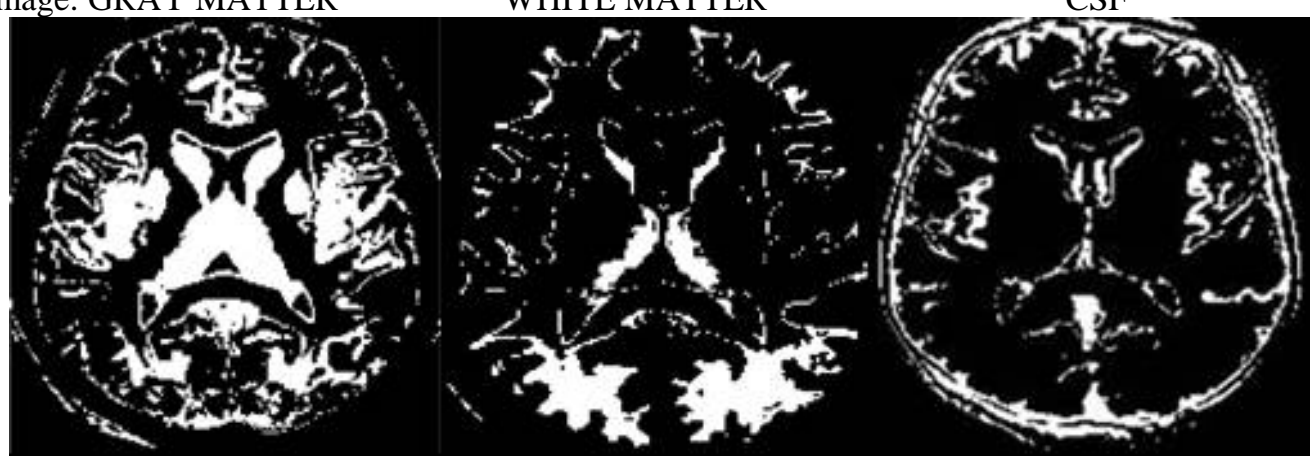

Figure A-19: Comparison of tissues between FCM and SPM segmentation of N3. 
Normal subject (N4): Comparison between FCM and SPM segmented images.

FCM:

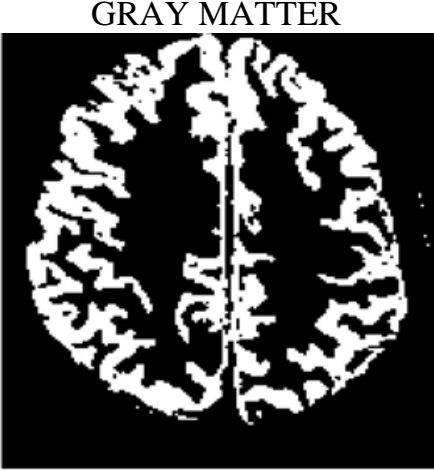

WHITE MATTER
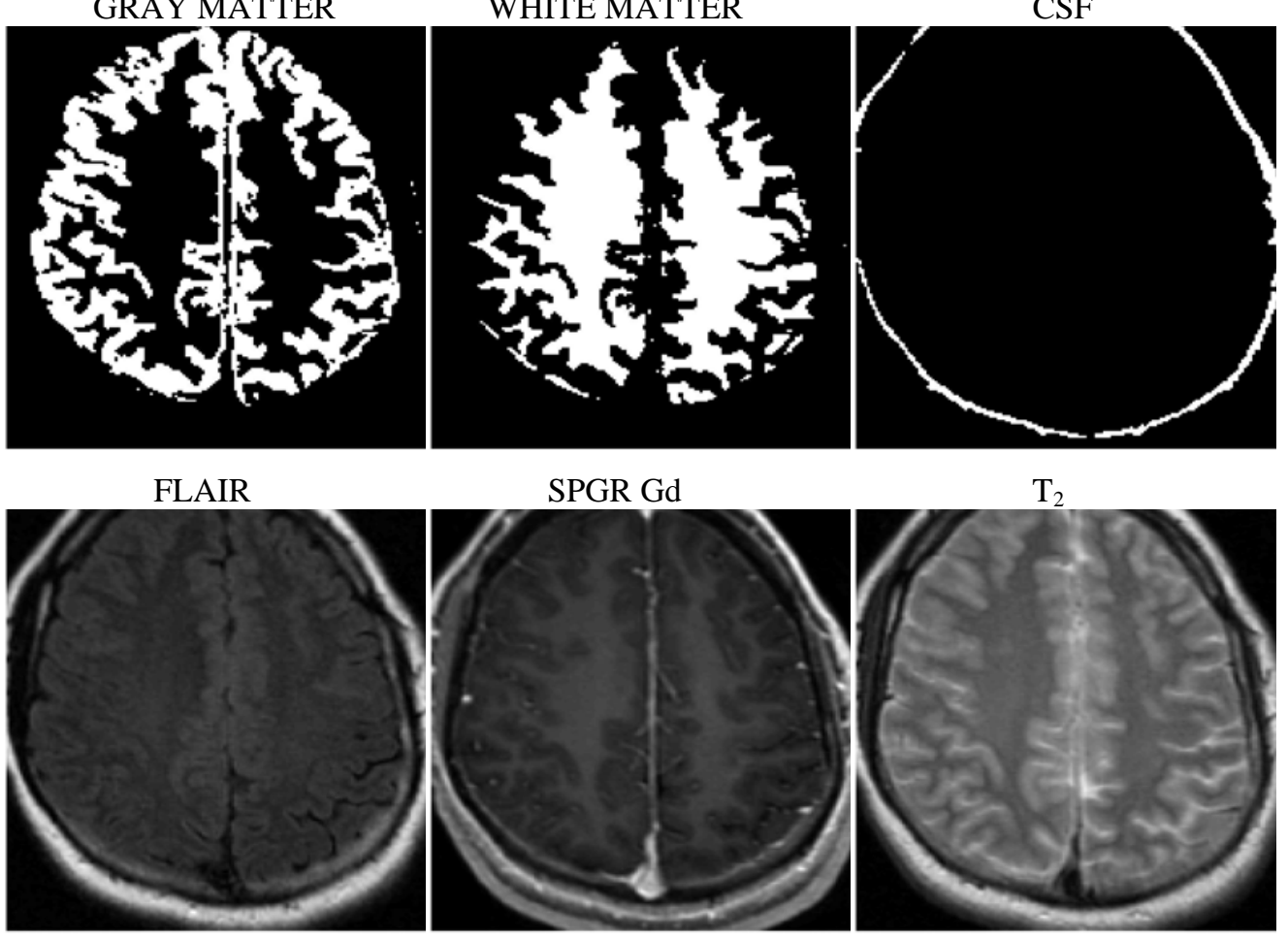

SPM:

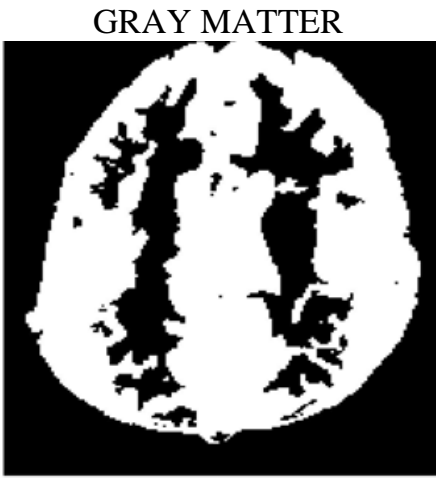

WHITE MATTER
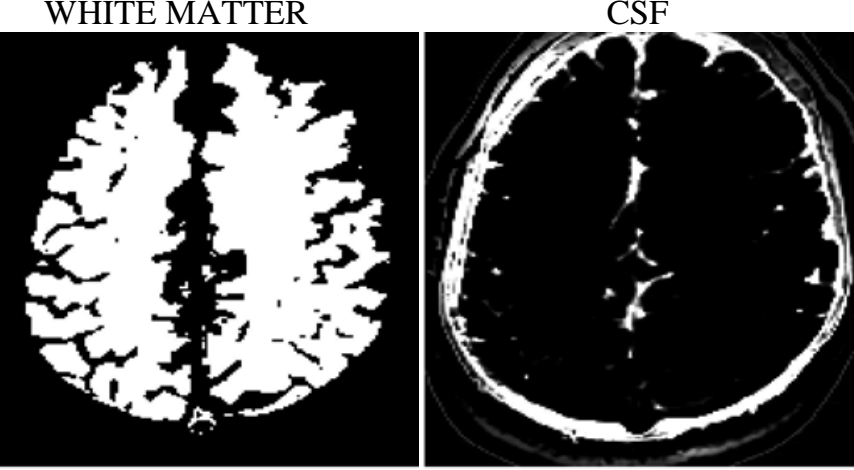

Diff. image: GRAY MATTER

WHITE MATTER

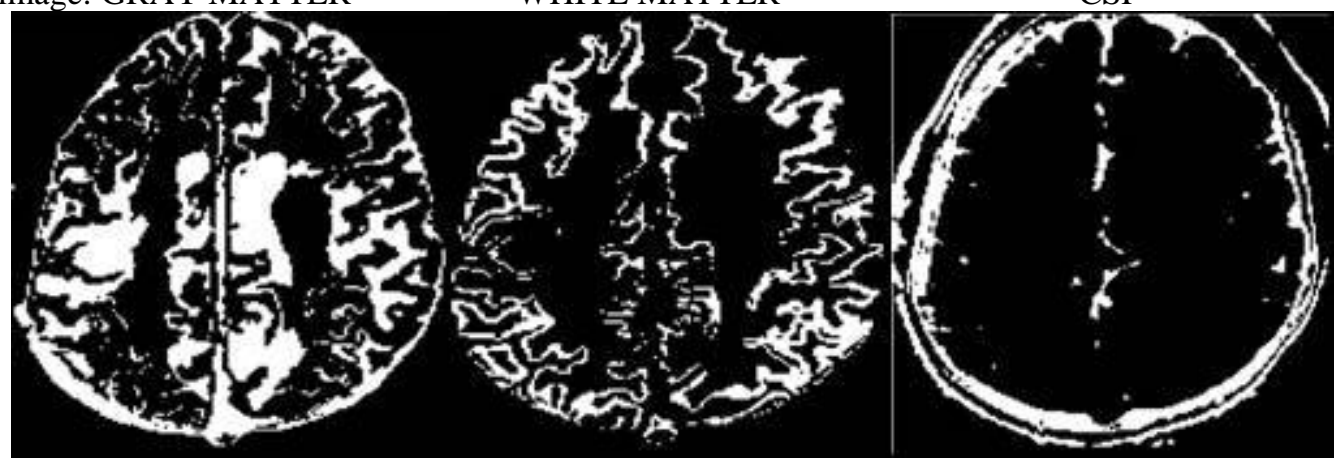

Figure A-20: Comparison of tissues between FCM and SPM segmentation of N4. 
Tumor patient (P1): Comparison between FCM and SPM segmented images

FCM:

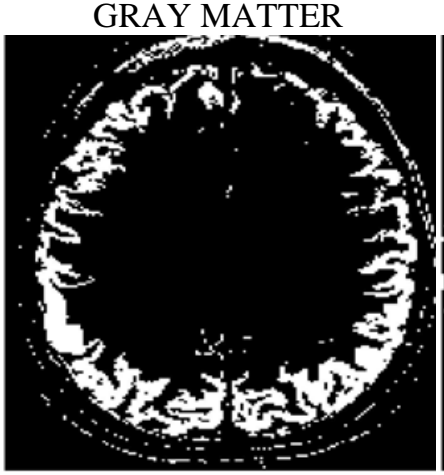

WHITE MATTER
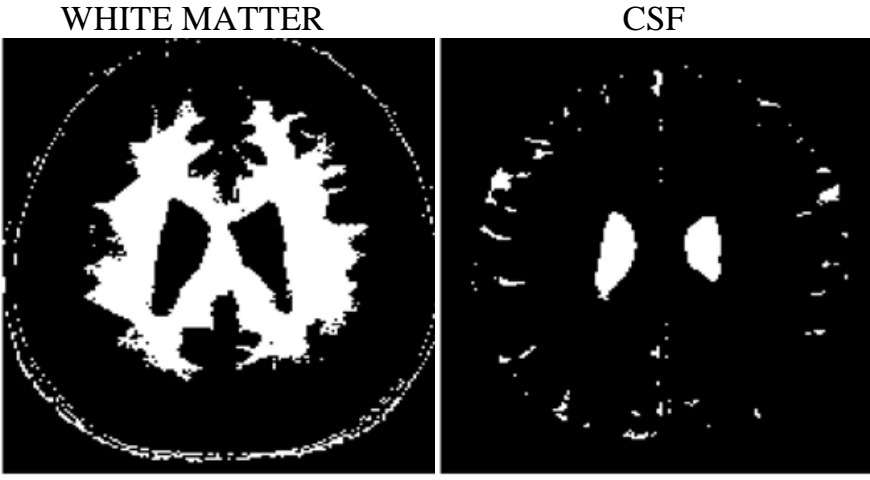

FLAIR

SPGR Gd
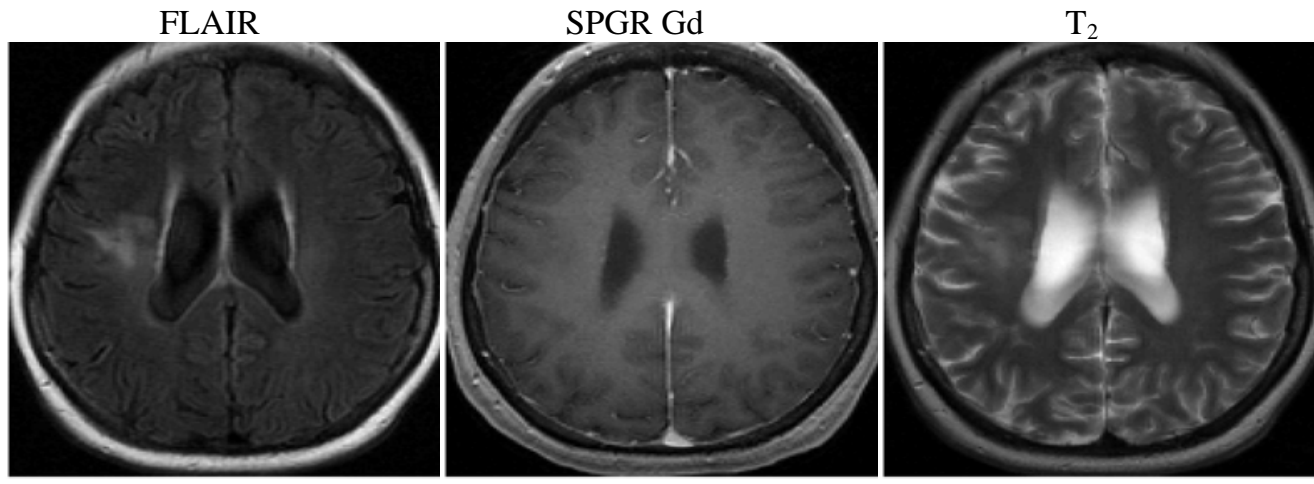

SPM:

GRAY MATTER

WHITE MATTER
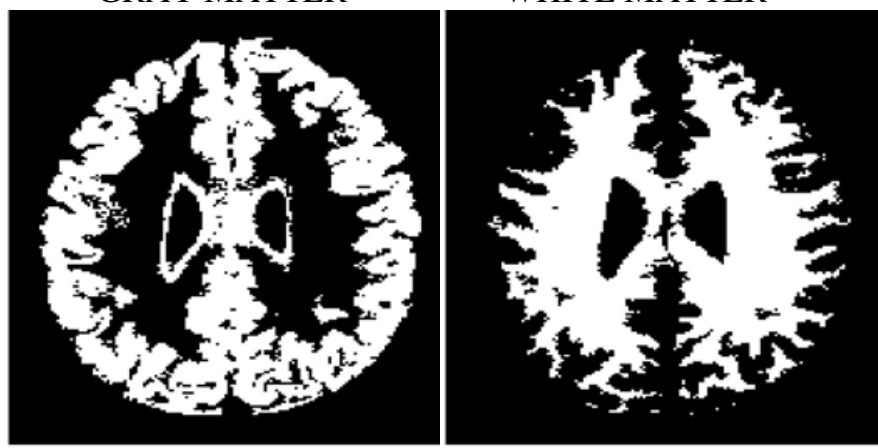

CSF

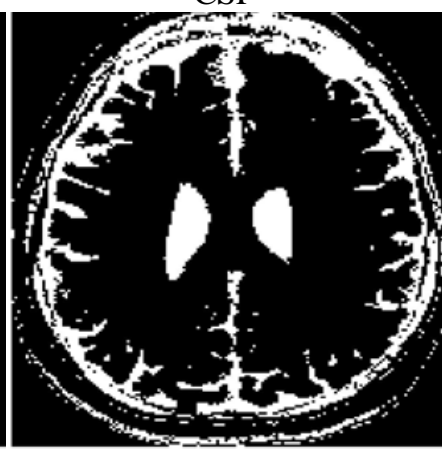

Diff. image: GRAY MATTER

WHITE MATTER

CSF

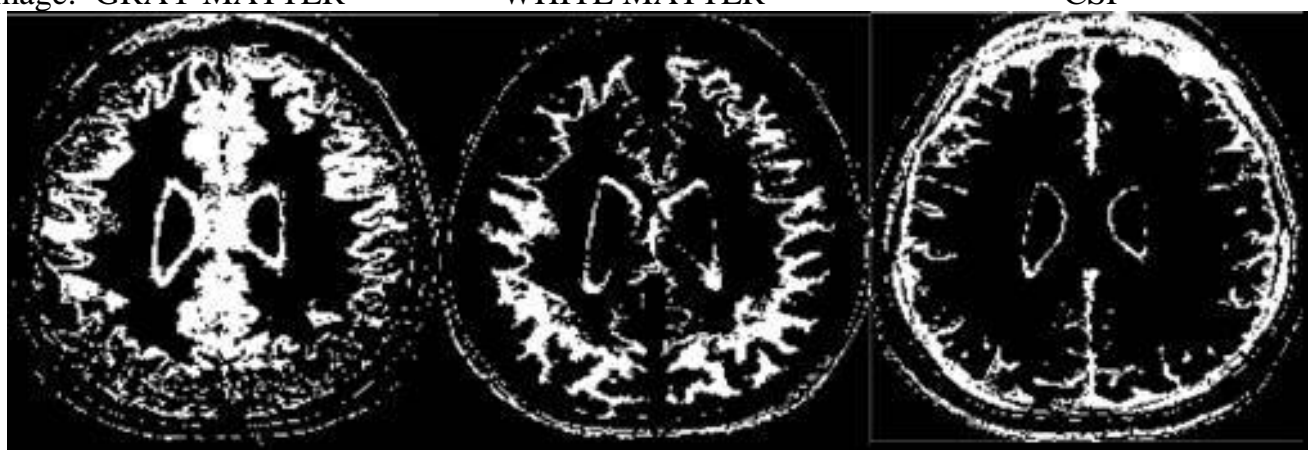

Figure A-21: Comparison of tissues between FCM and SPM segmentation of P1. 
Tumor patient (P2): Comparison between FCM and SPM segmented images.

FCM:

GRAY MATTER

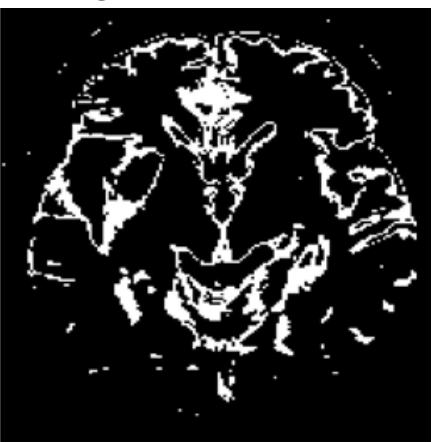

FLAIR

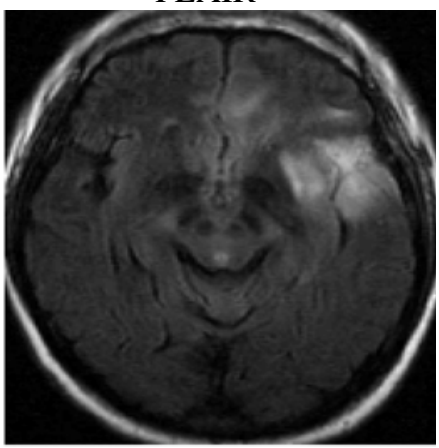

SPM:

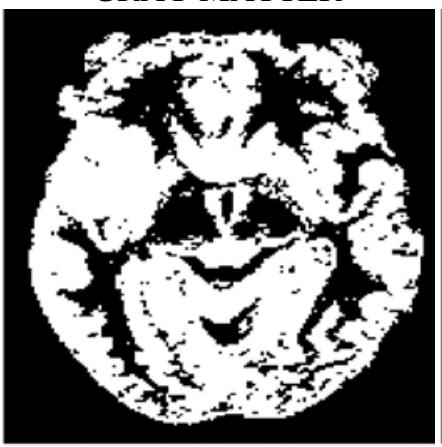

WHITE MATTER

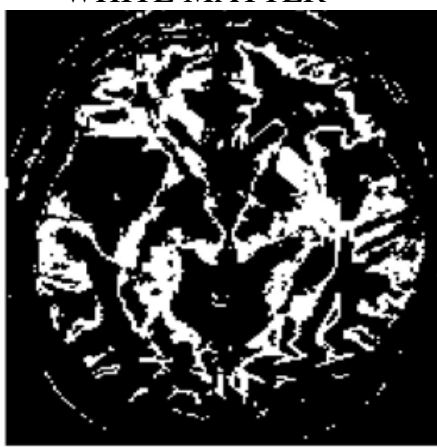

SPGR Gd

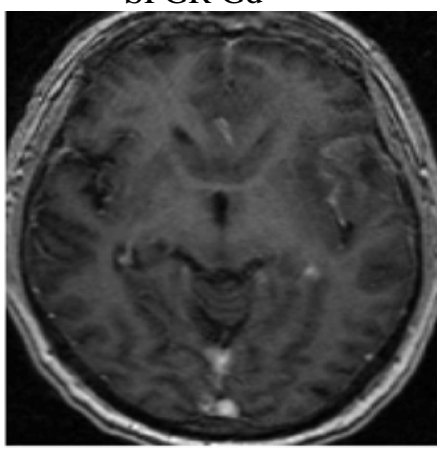

WHITE MATTER

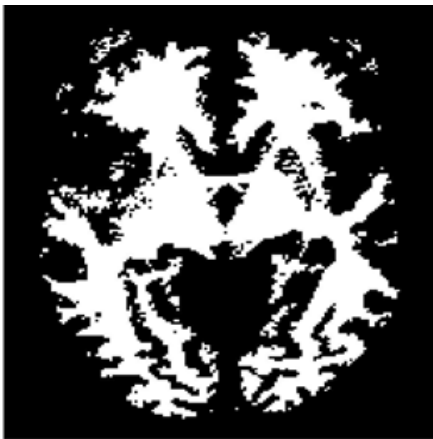

WHITE MATTER

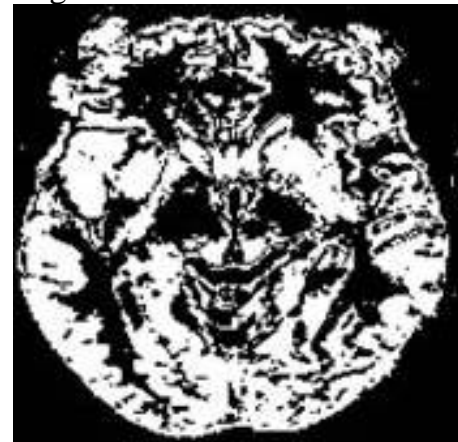

CSF

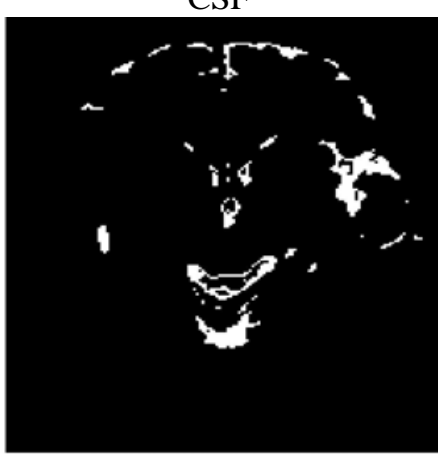

$\mathrm{T}_{2}$

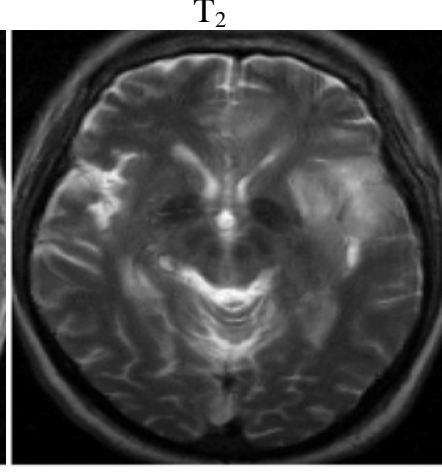

$\mathrm{CSF}$

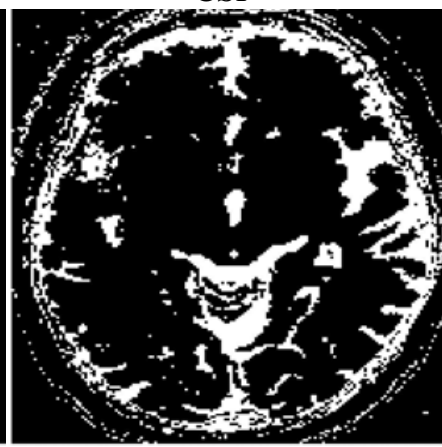

CSF

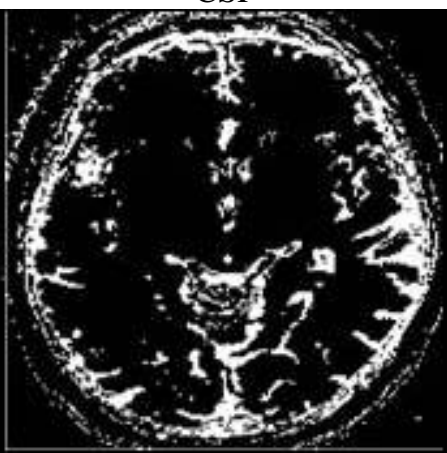

Figure A-22: Comparison of tissues between FCM and SPM segmentation of P2. 
Tumor patient (P3): Comparison between FCM and SPM segmented images.

FCM:

GRAY MATTER
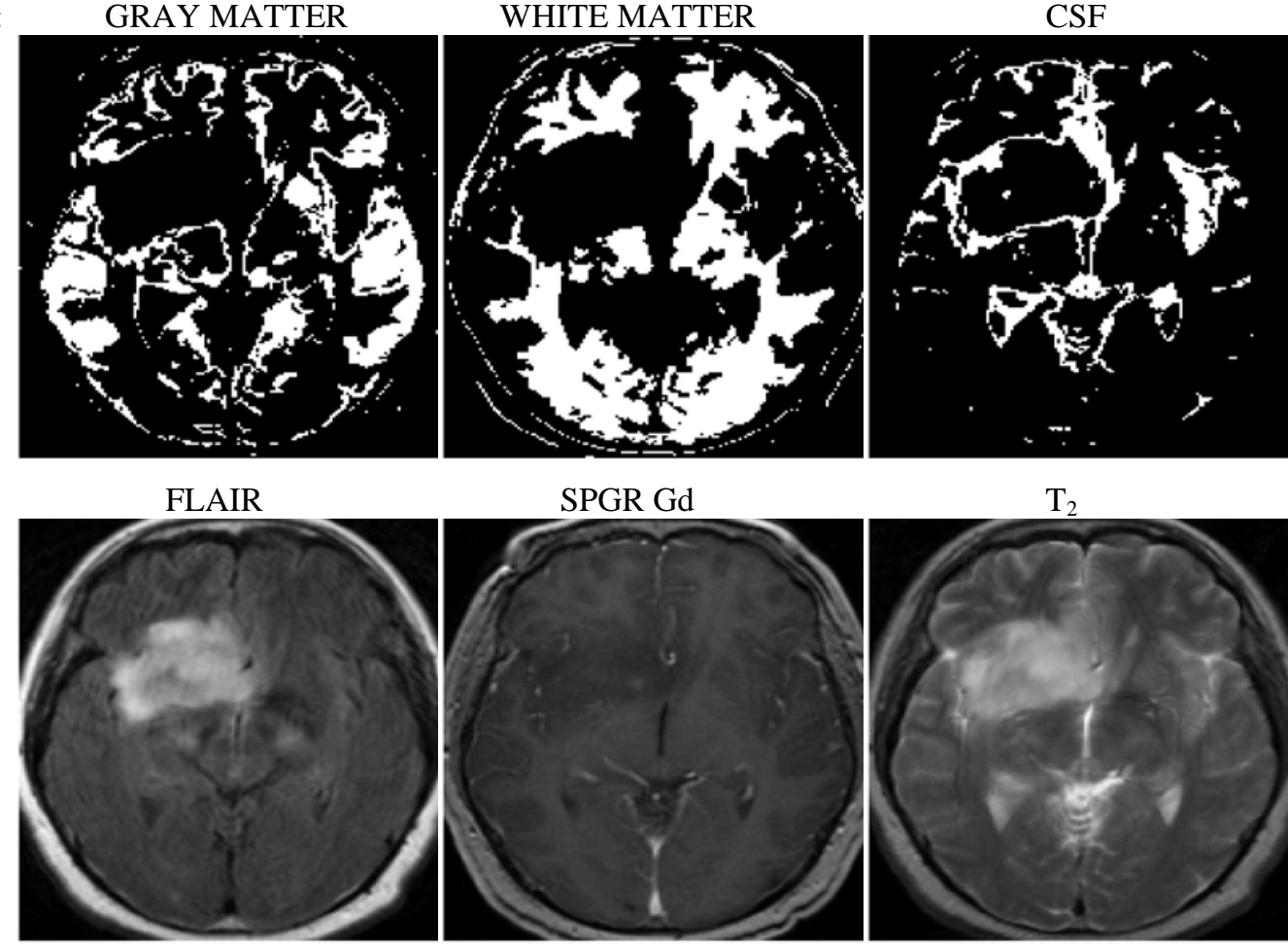

SPGR Gd
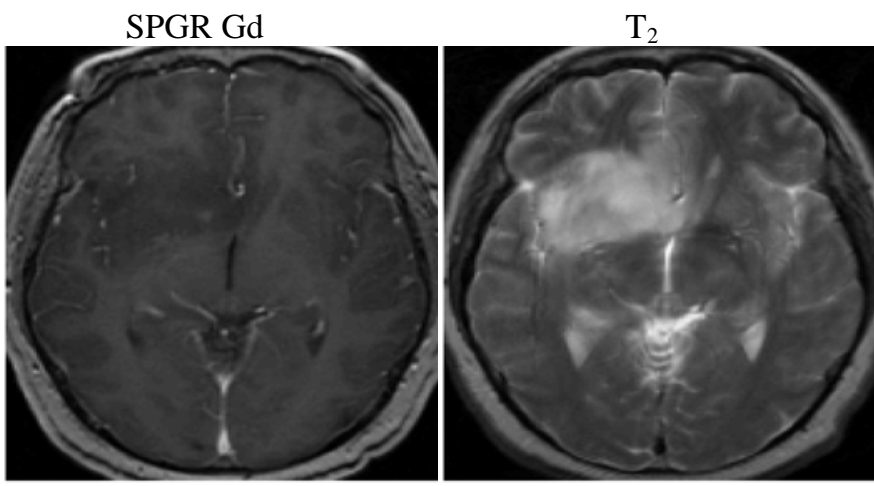

SPM:

GRAY MATTER

WHITE MATTER
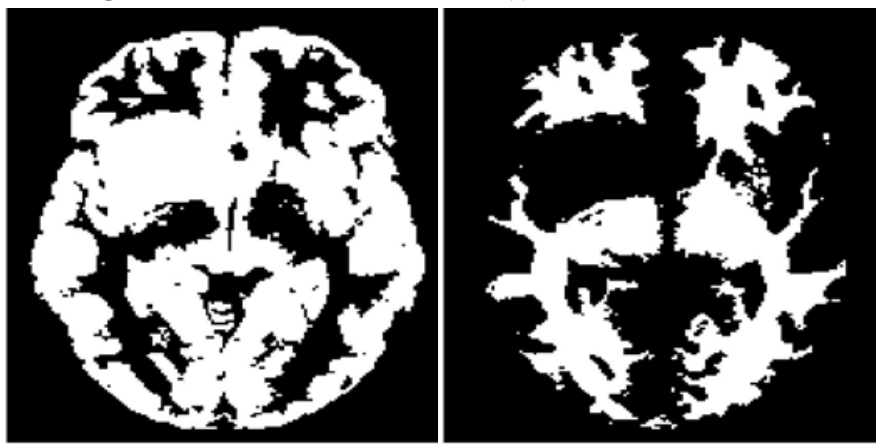

$\mathrm{CSF}$

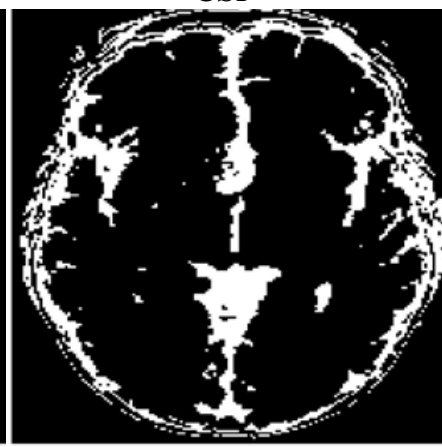

Diff. image: GRAY MATTER

WHITE MATTER

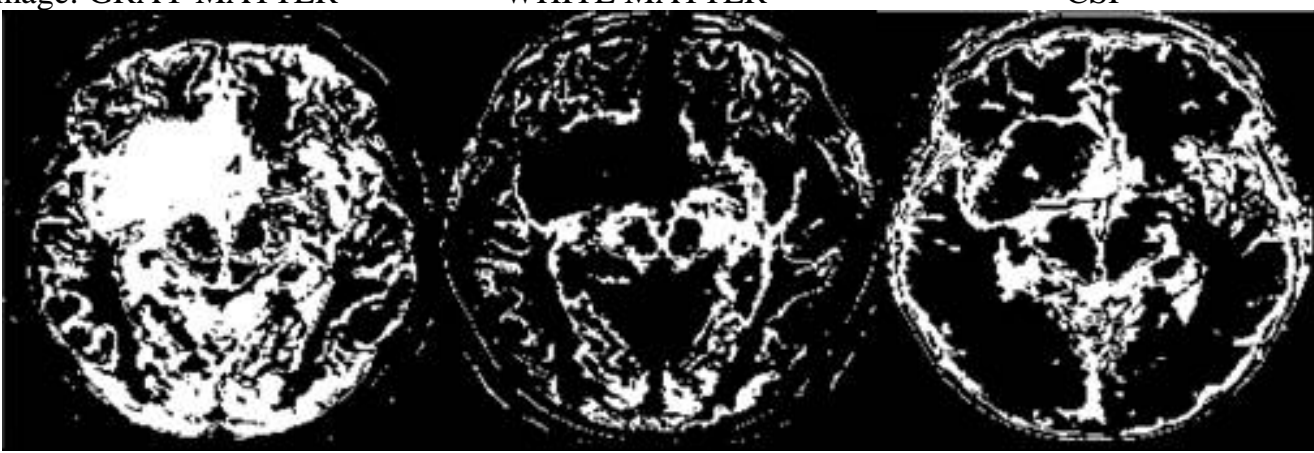

Figure A-23: Comparison of tissues between FCM and SPM segmentation of P3. 
Tumor patient (P4): Comparison between FCM and SPM segmented images.

FCM:

GRAY MATTER

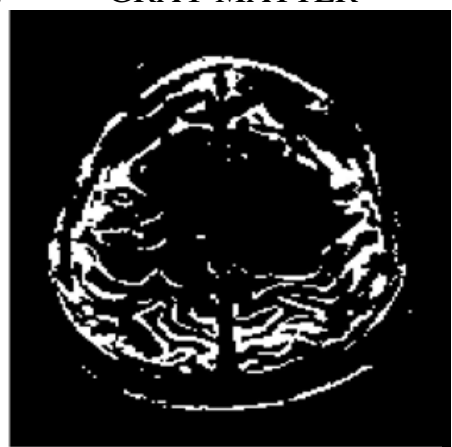

FLAIR

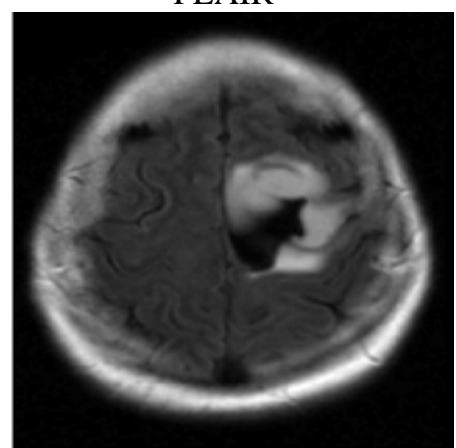

SPM:

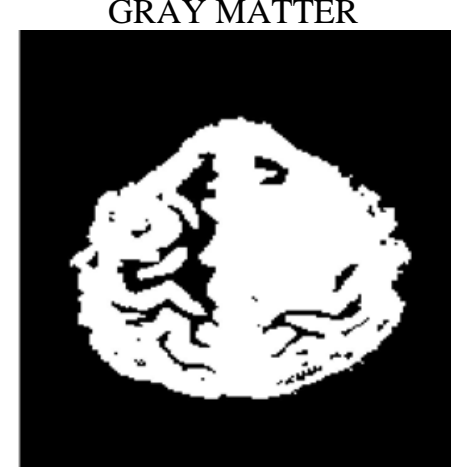

Diff. image: GRAY MATTER

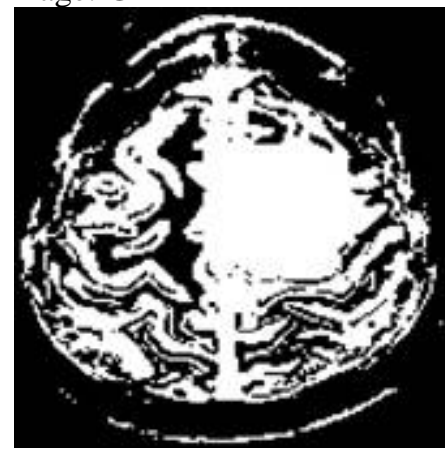

WHITE MATTER

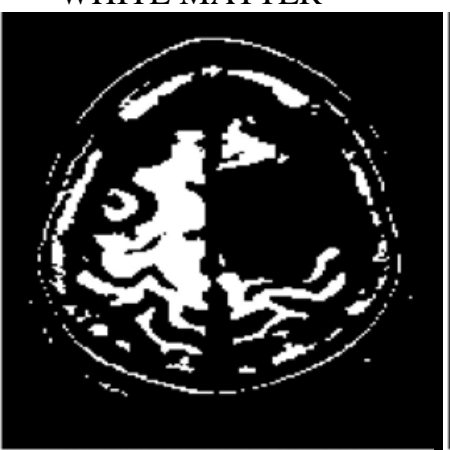

SPGR Gd

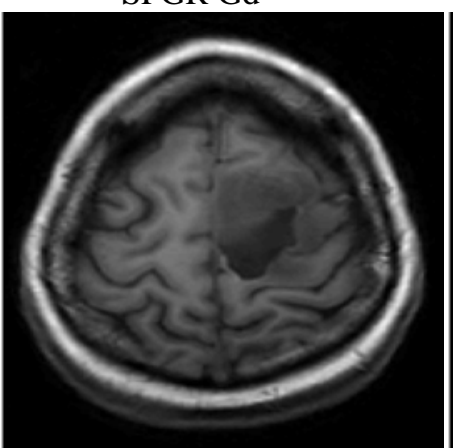

WHITE MATTER
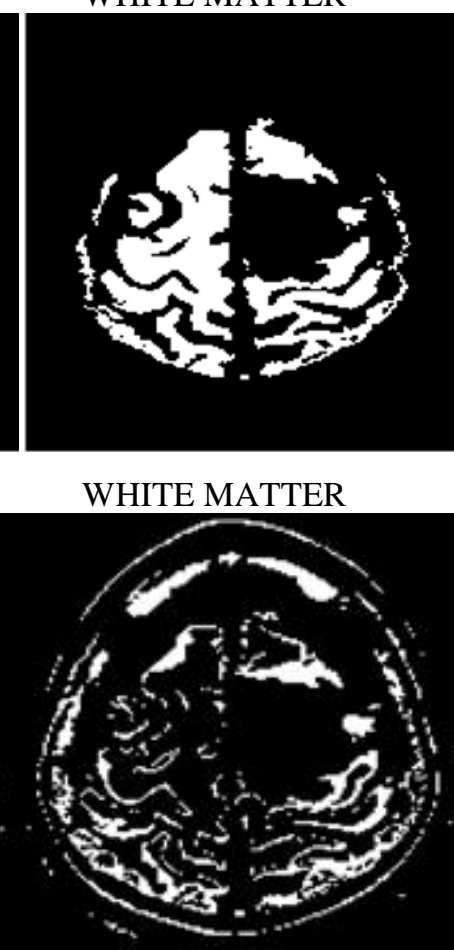

CSF

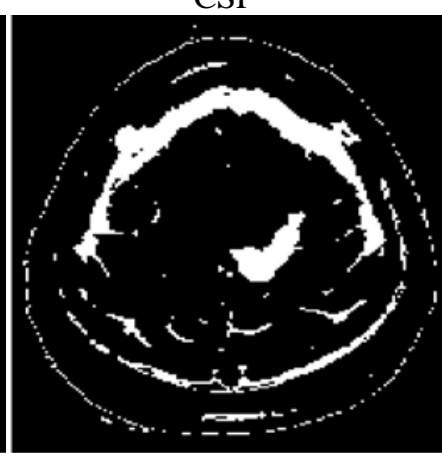

Figure A-24: Comparison of tissues between FCM and SPM segmentation of P4. 
Normal subject (N1): Inputs to FCM (FLAIR, T2, SPGR, SPGRGD, DWI)
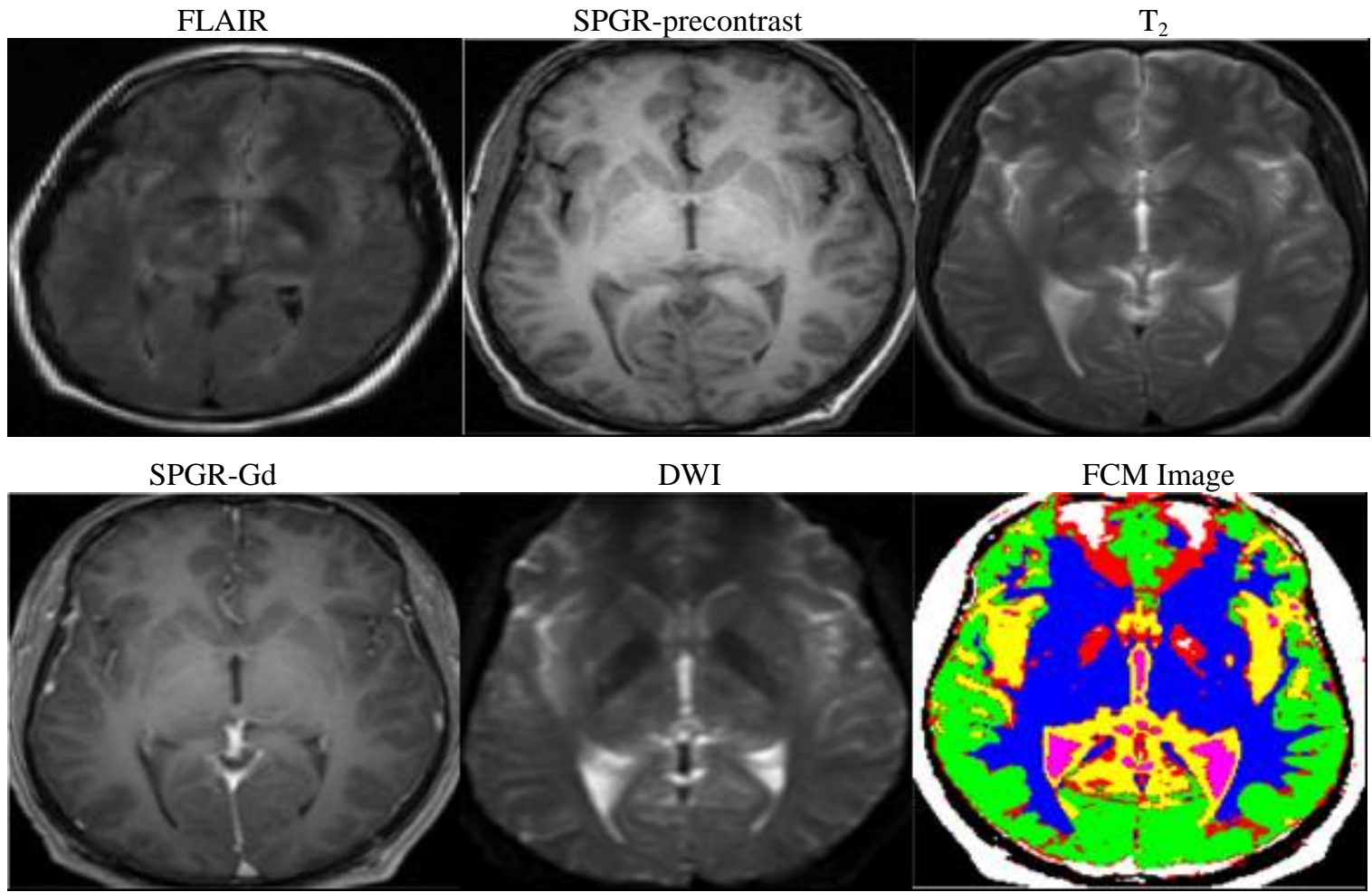

Figure A-25: MRI volume data sets segmented into six classes (tissue) types.
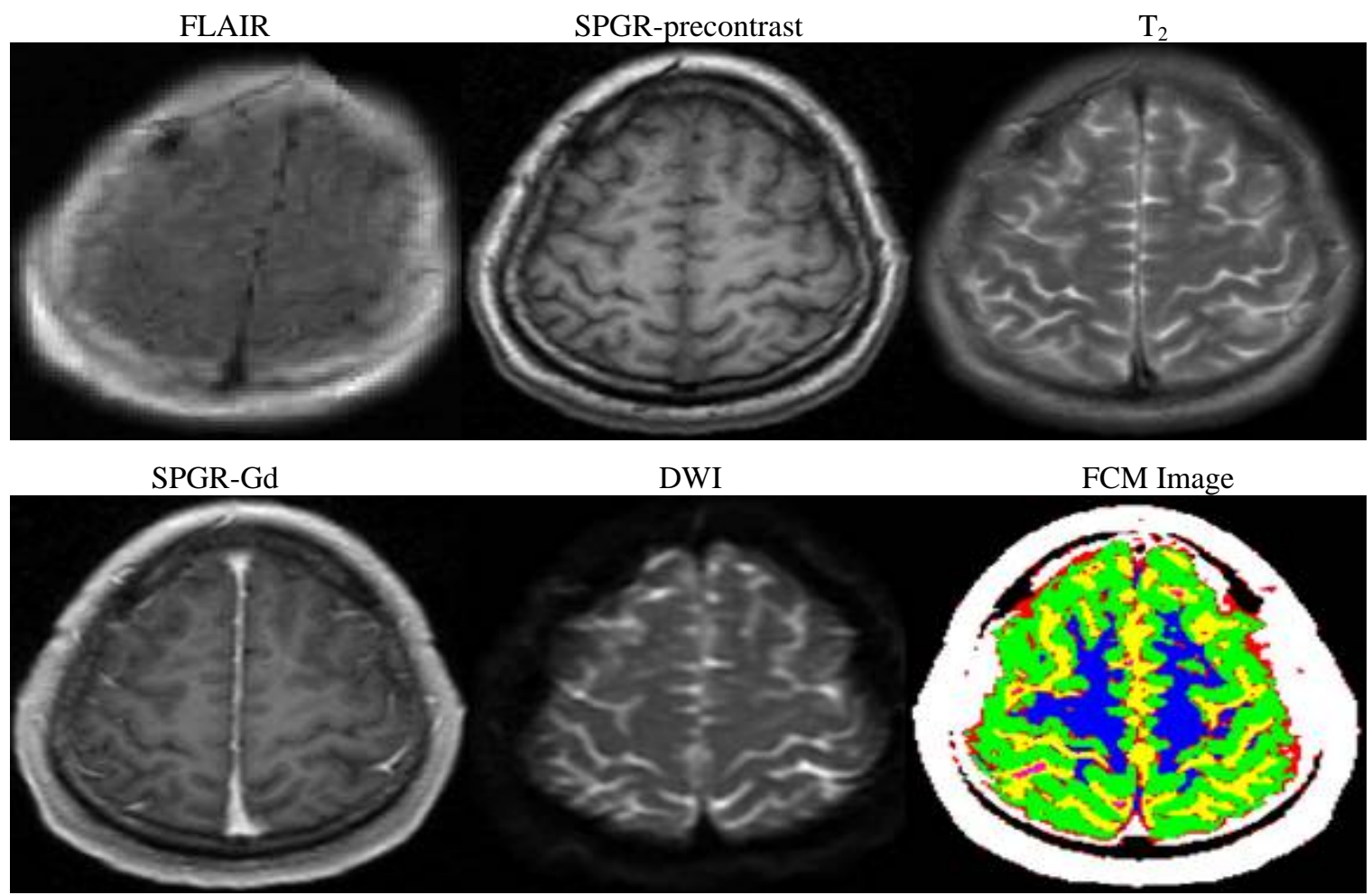

Figure A-26: MRI volume data sets segmented into six classes (tissue) types. 
Normal subject (N2): Inputs to FCM (FLAIR, T ${ }_{2}$, SPGR, SPGRGD, DWI)
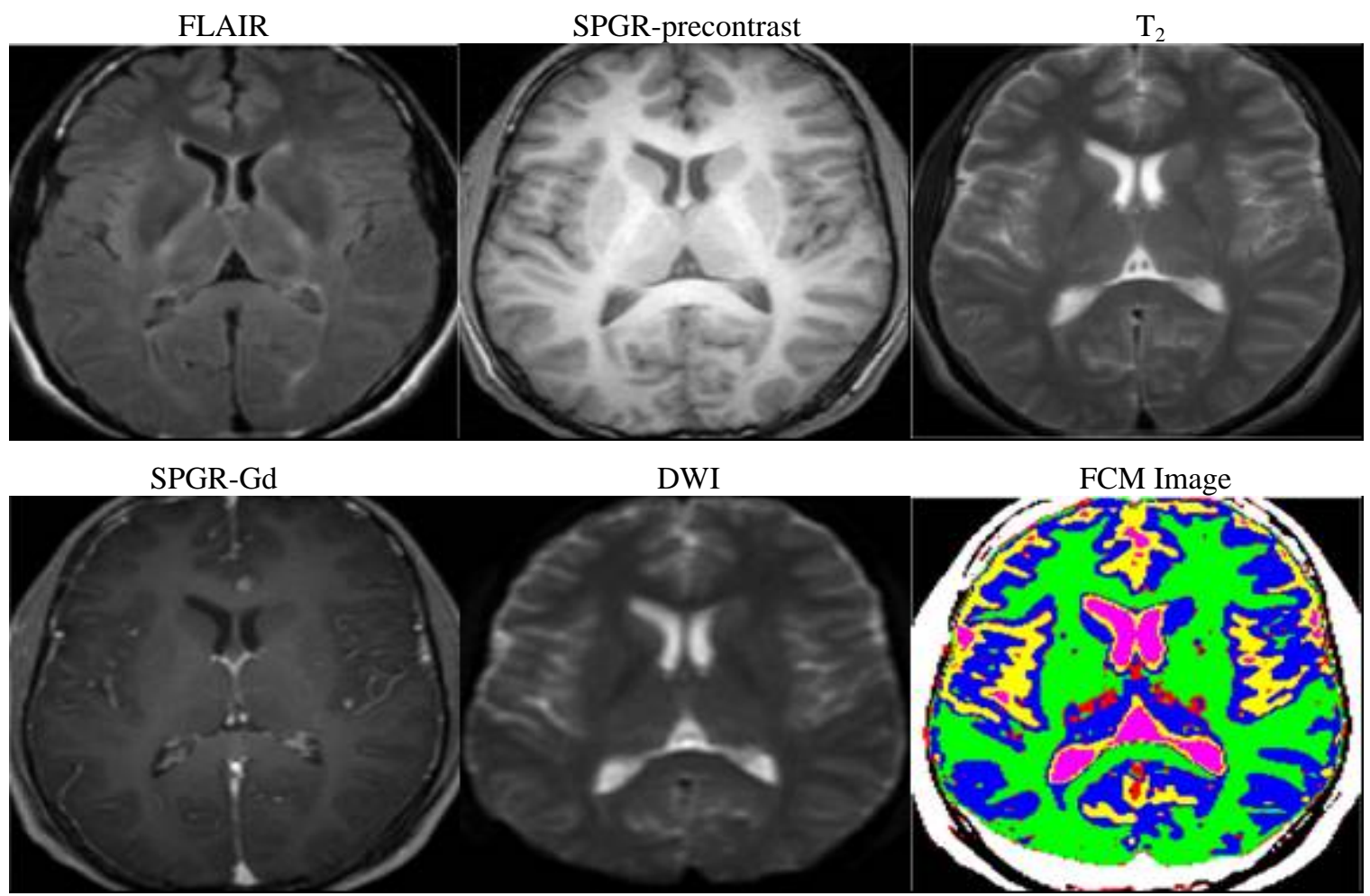

Figure A-27: MRI volume data sets segmented into six classes (tissue) types.
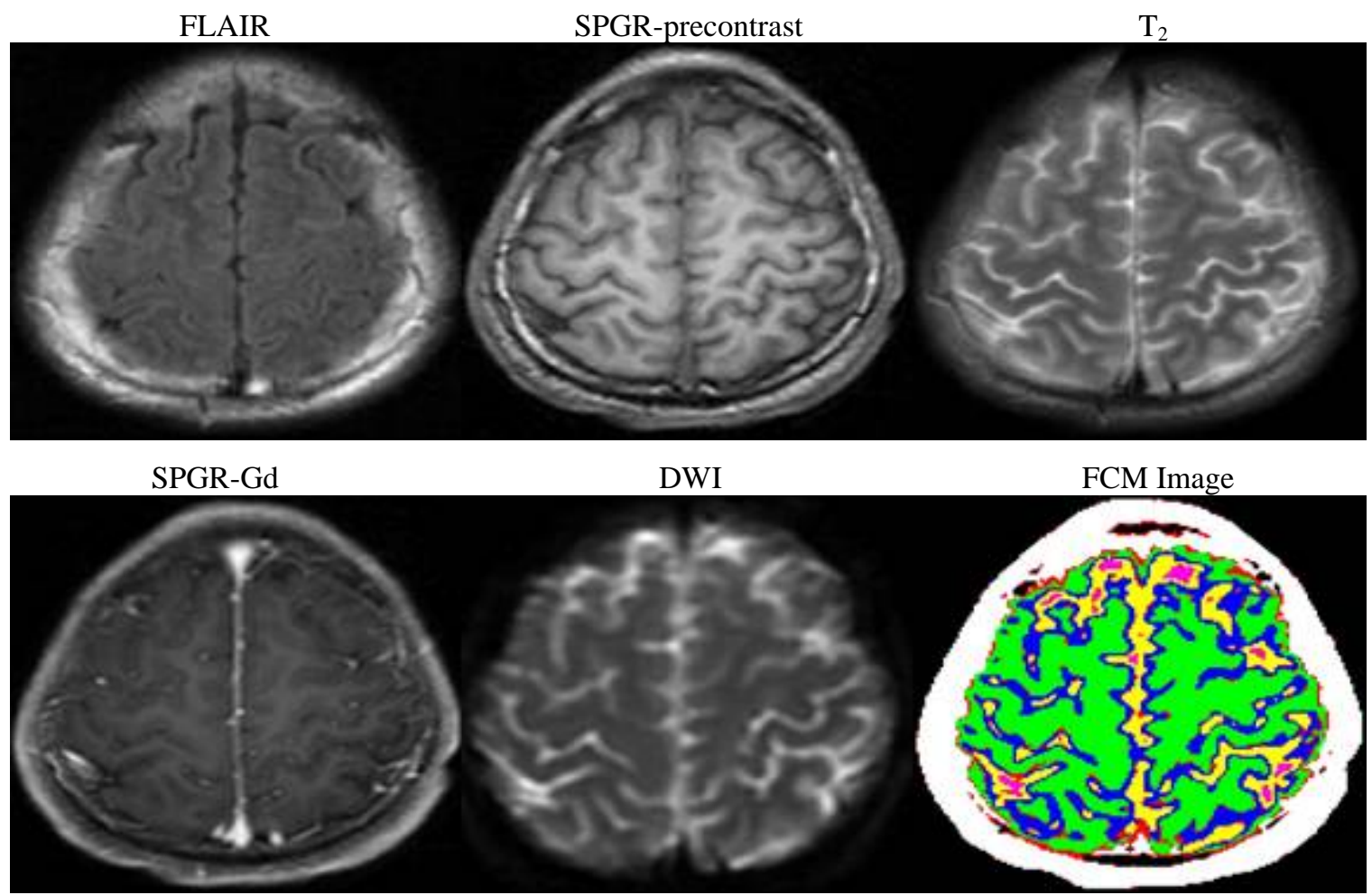

Figure A-28: MRI volume data sets segmented into six classes (tissue) types. 
Normal subject (N3): Inputs to FCM (FLAIR, T ${ }_{2}$, SPGR, SPGRGD, DWI)
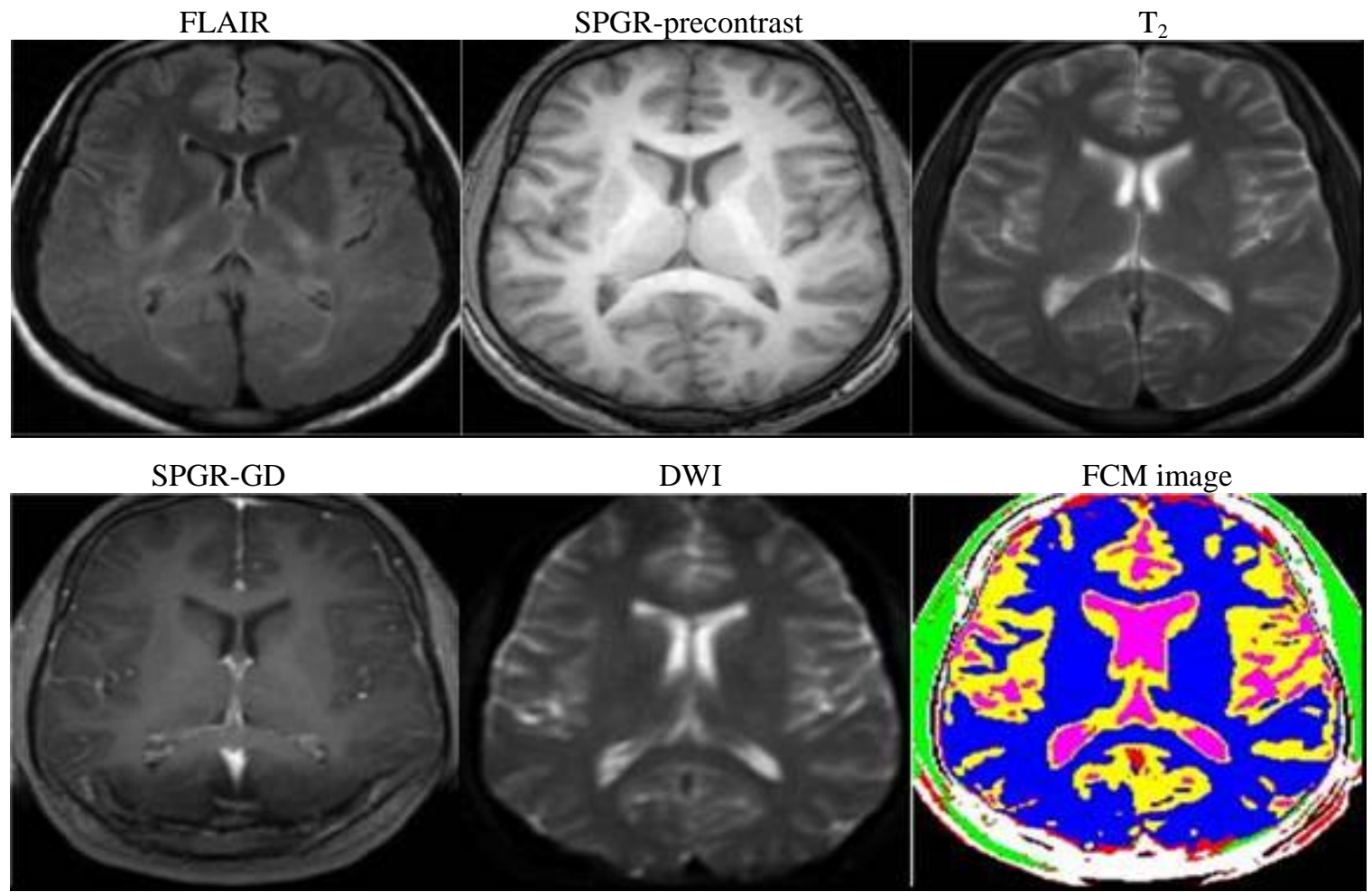

Figure A-29: MRI volume data sets segmented into six classes (tissue) types.
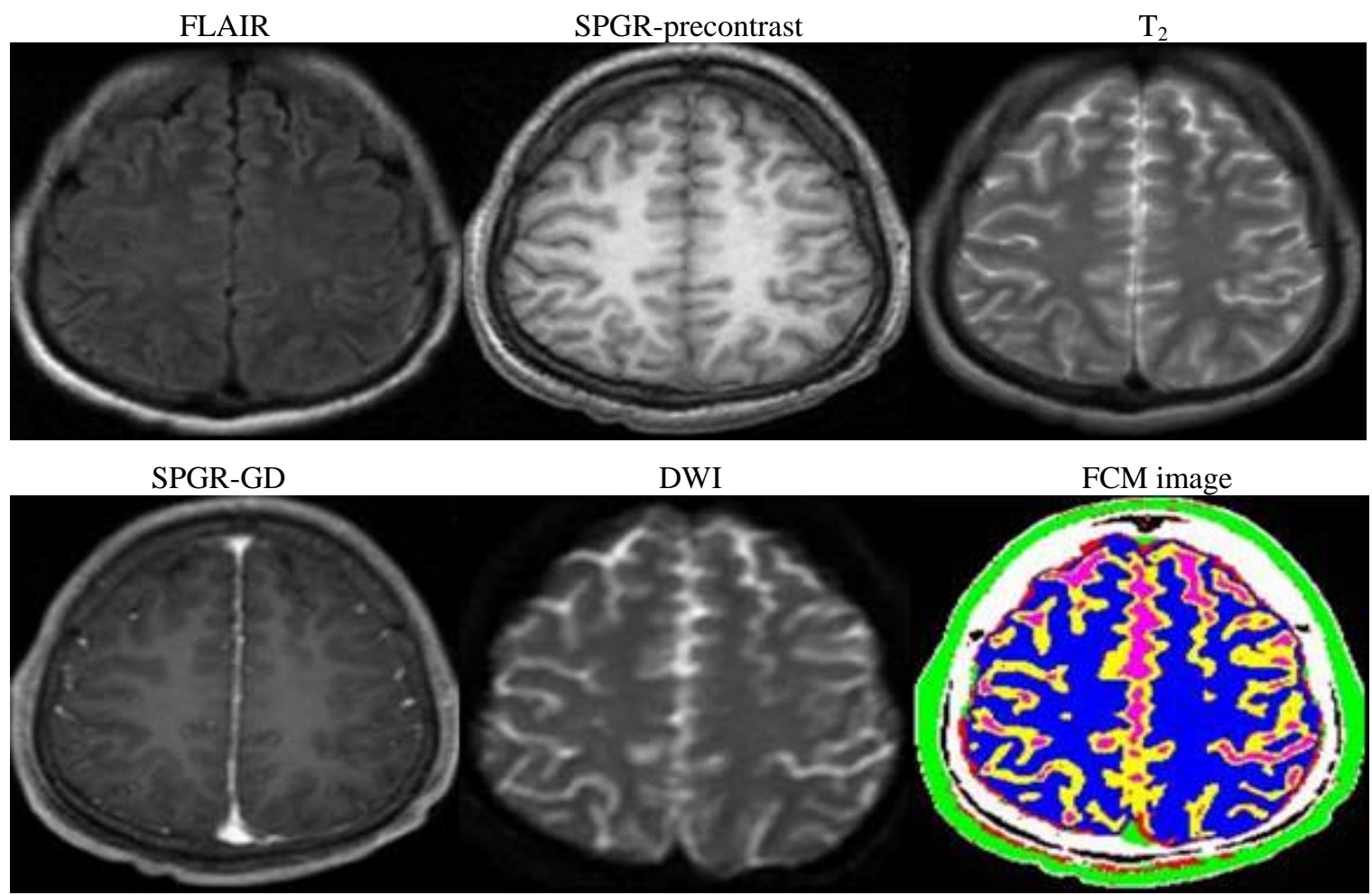

Figure A-30: MRI volume data sets segmented into six classes (tissue) types. 
Normal subject (N4): Inputs to FCM (FLAIR, T2, SPGR, SPGRGD, DWI)
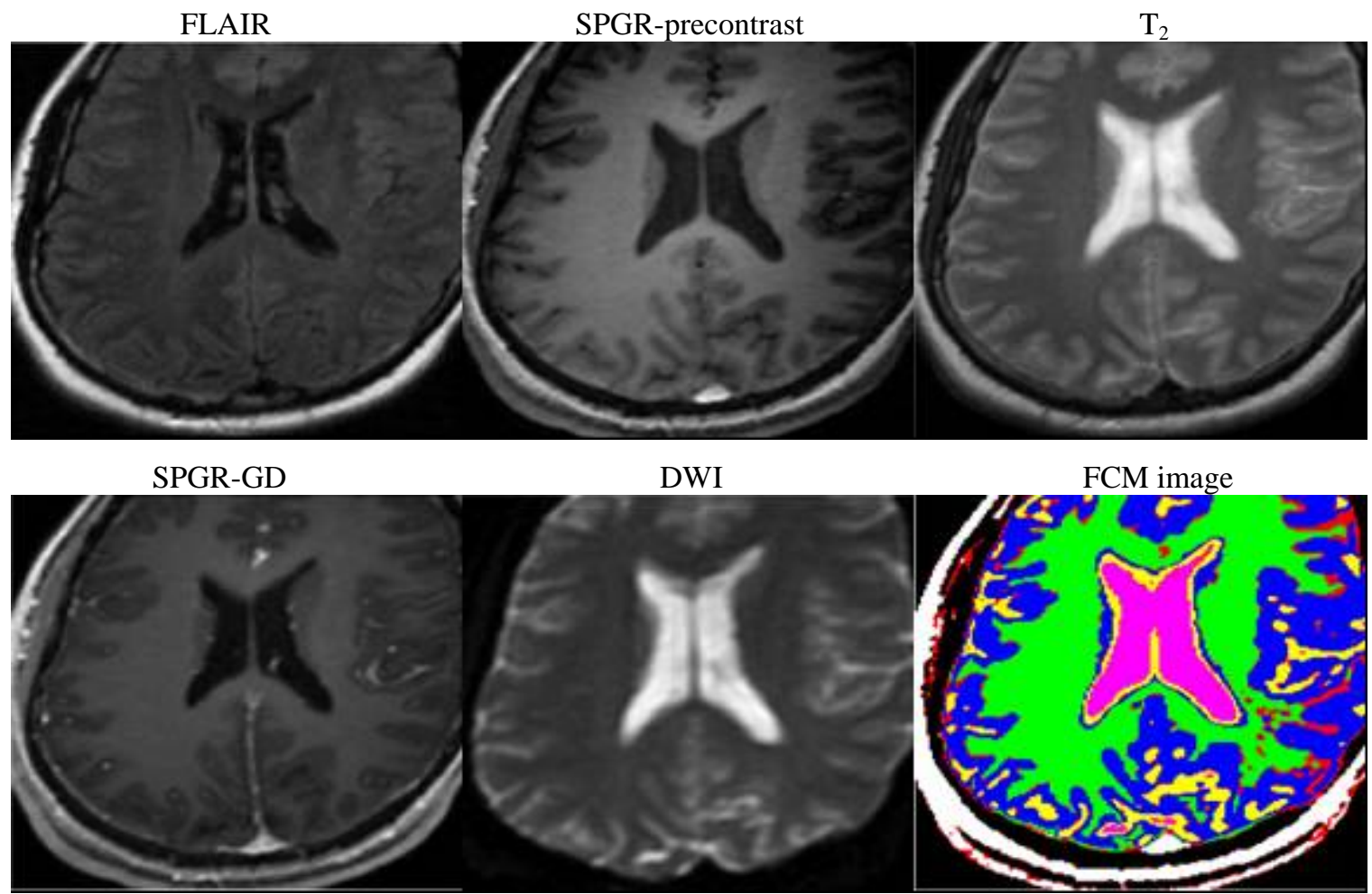

Figure A-31: MRI volume data sets segmented into six classes (tissue) types.
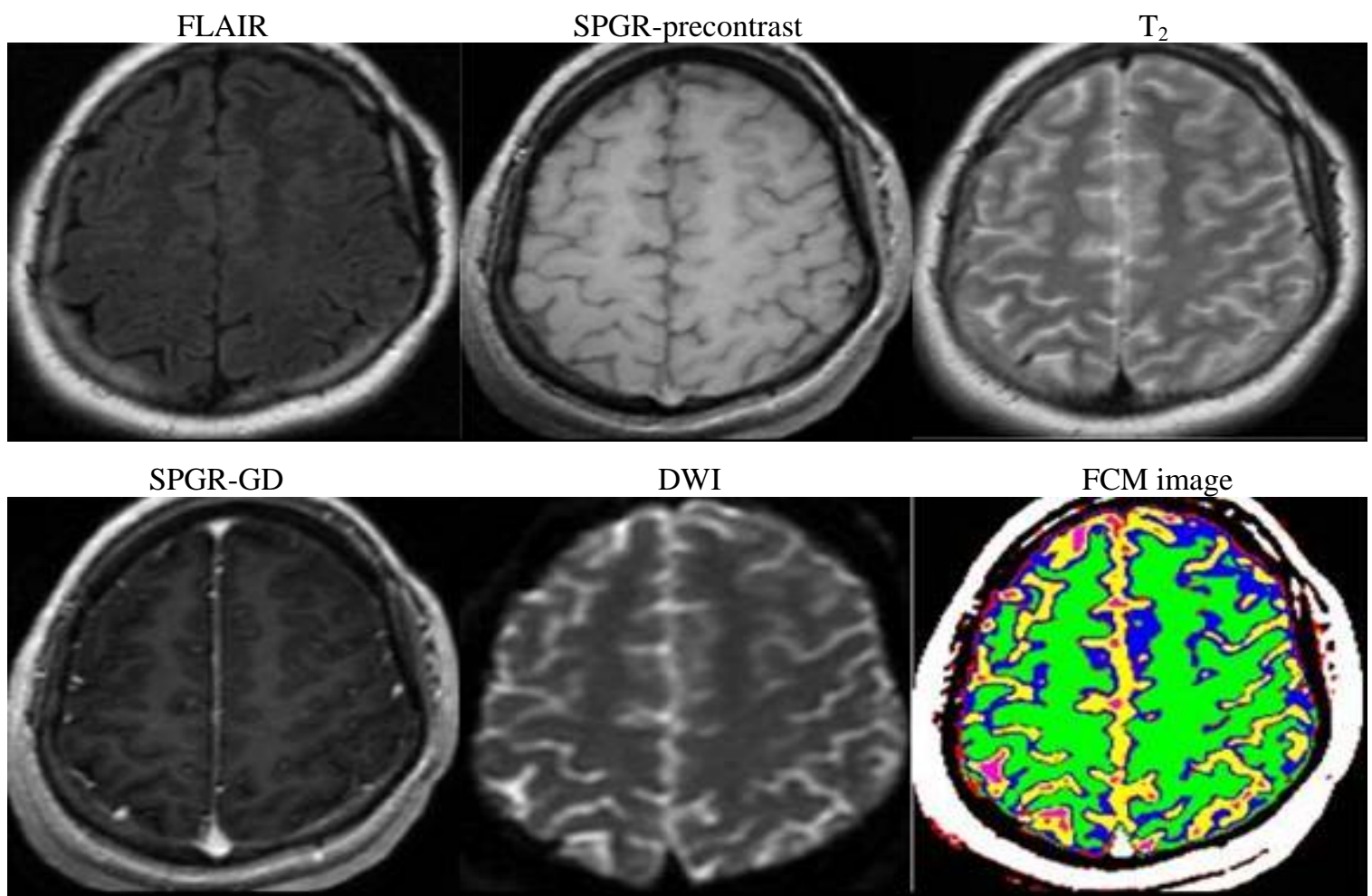

Figure A-32: MRI volume data sets segmented into six classes (tissue) types. 
Tumor patient (P1): Inputs to FCM (FLAIR, T2, SPGR, SPGRGD, DWI)
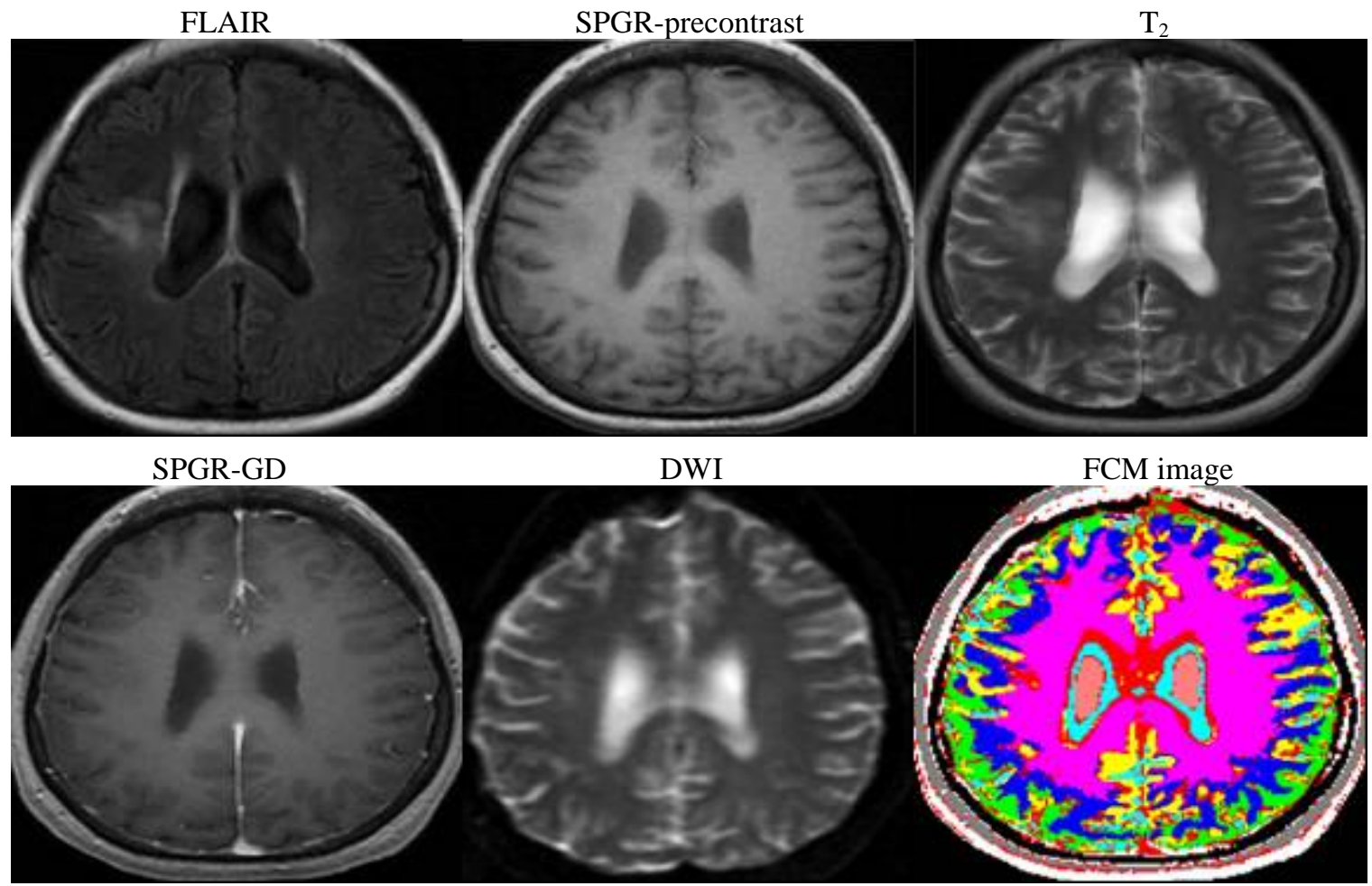

Figure A-33: MRI volume data sets segmented into nine classes (tissue) types.

Tumor patient (P2): Inputs to FCM (FLAIR, T2, SPGR, SPGRGD, DWI)
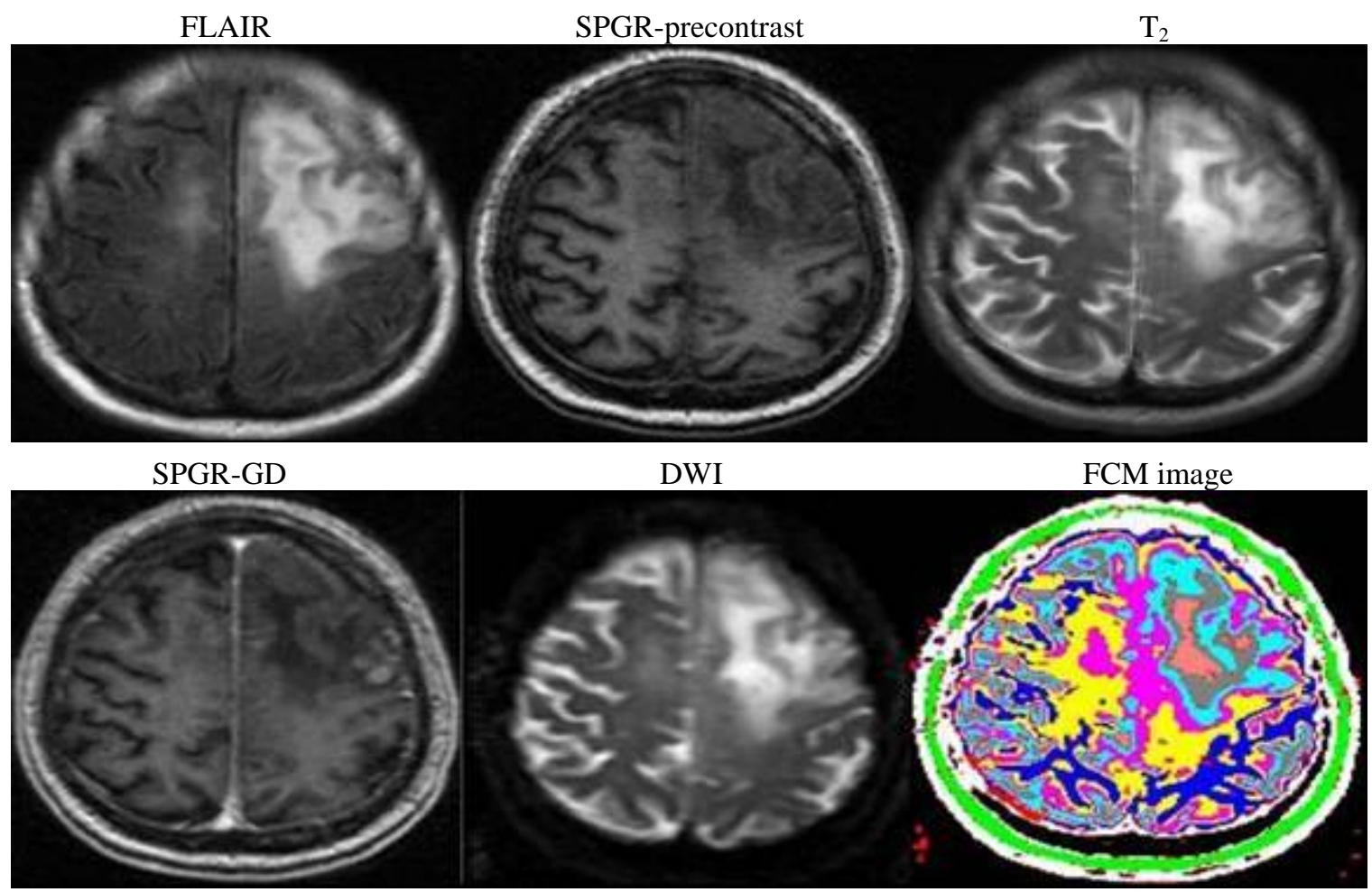

Figure A-34: MRI volume data sets segmented into nine classes (tissue) types. 
Tumor patient (P3): Inputs to FCM (FLAIR, T2, SPGR, SPGRGD, DWI)
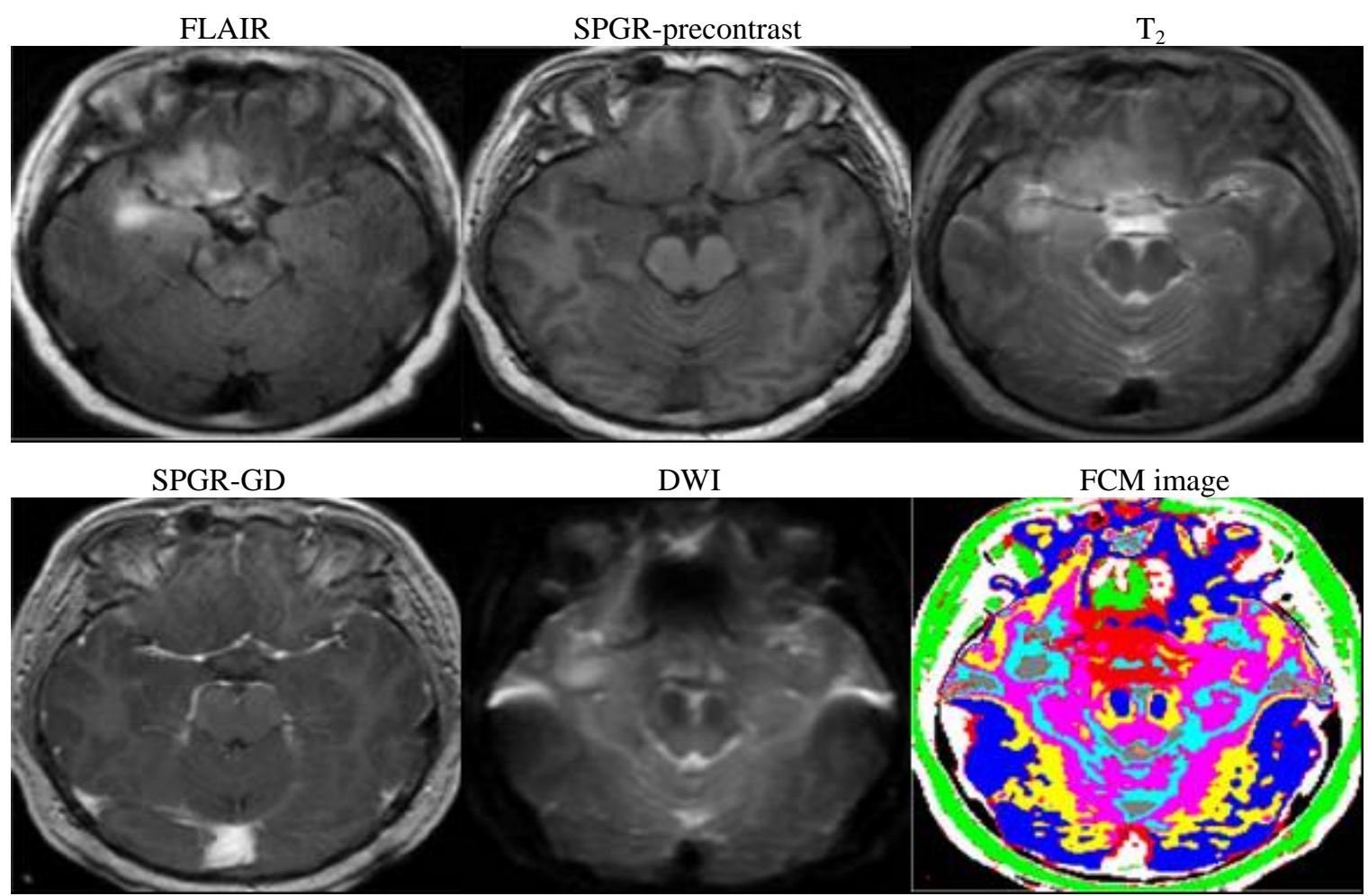

Figure A-35: MRI volume data sets segmented into nine classes (tissue) types. 
Normal subject (N1): Comparison between FCM and SPM segmented images using five feature vectors.

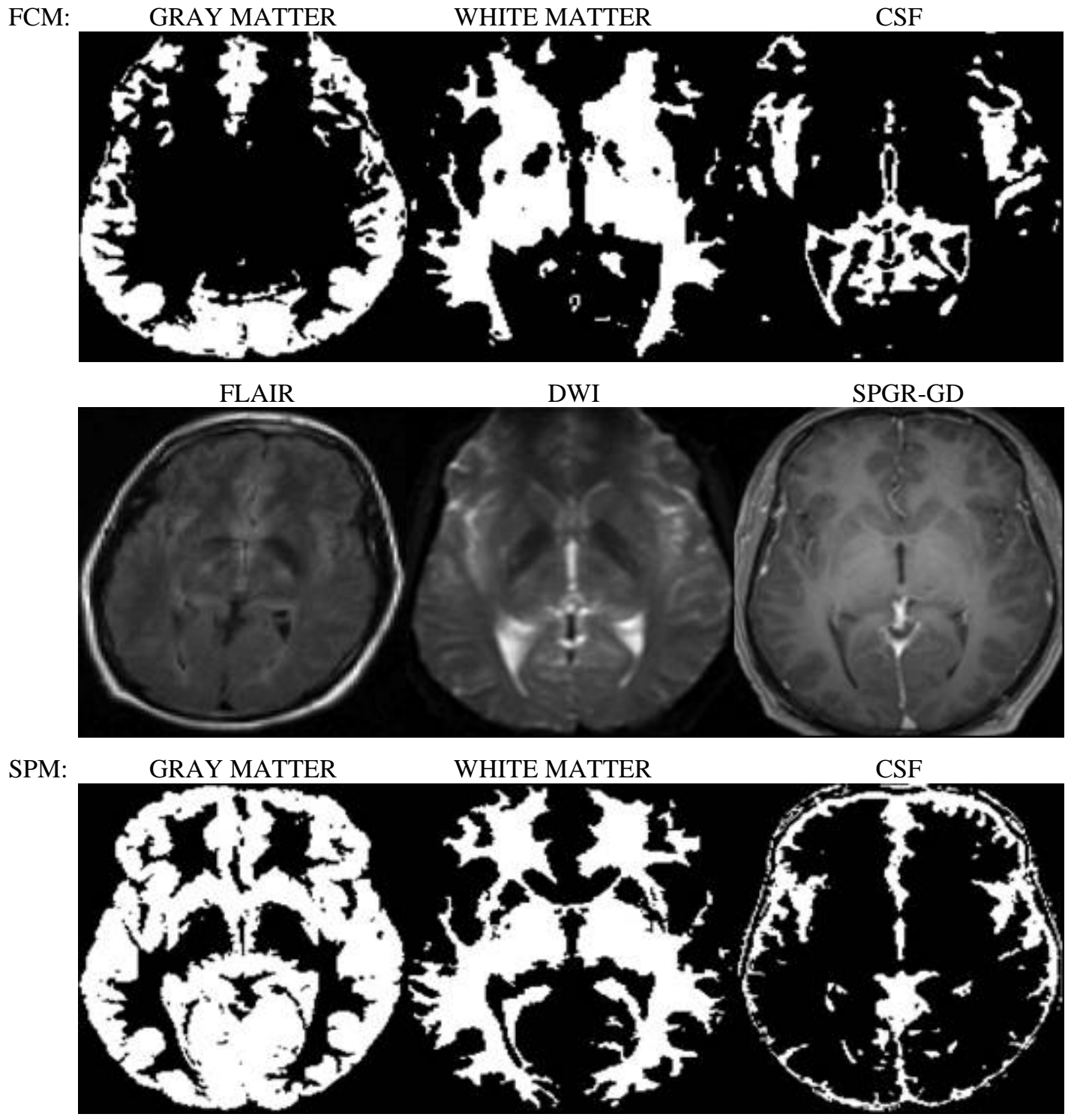

Diff. image: GRAY MATTER WHITE MATTER CSF

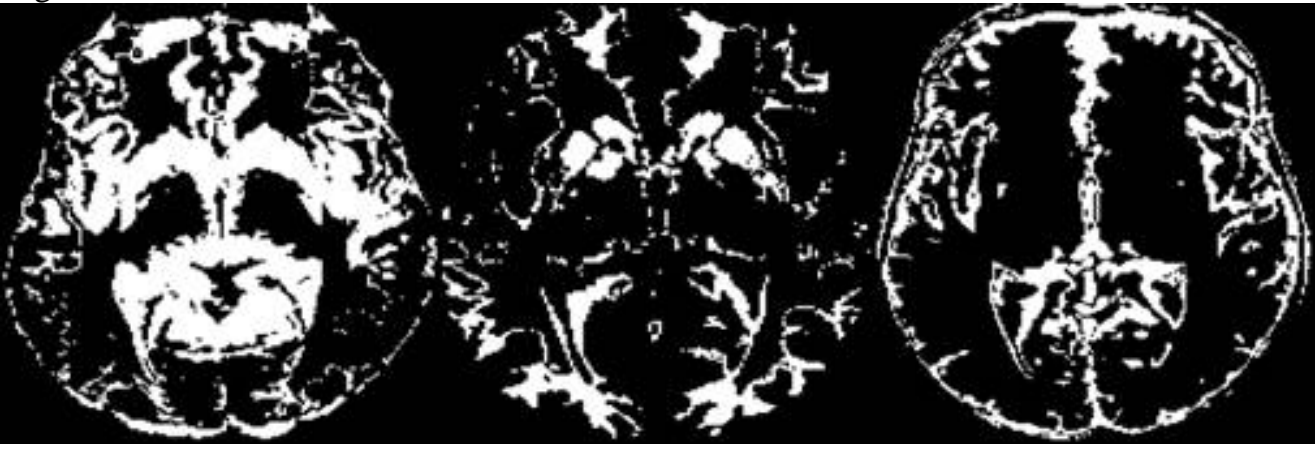

Figure A-36 Comparison of FCM and SPM segmented images using five feature vectors 
Normal subject (N2): Comparison between FCM and SPM segmented images using five feature vectors.

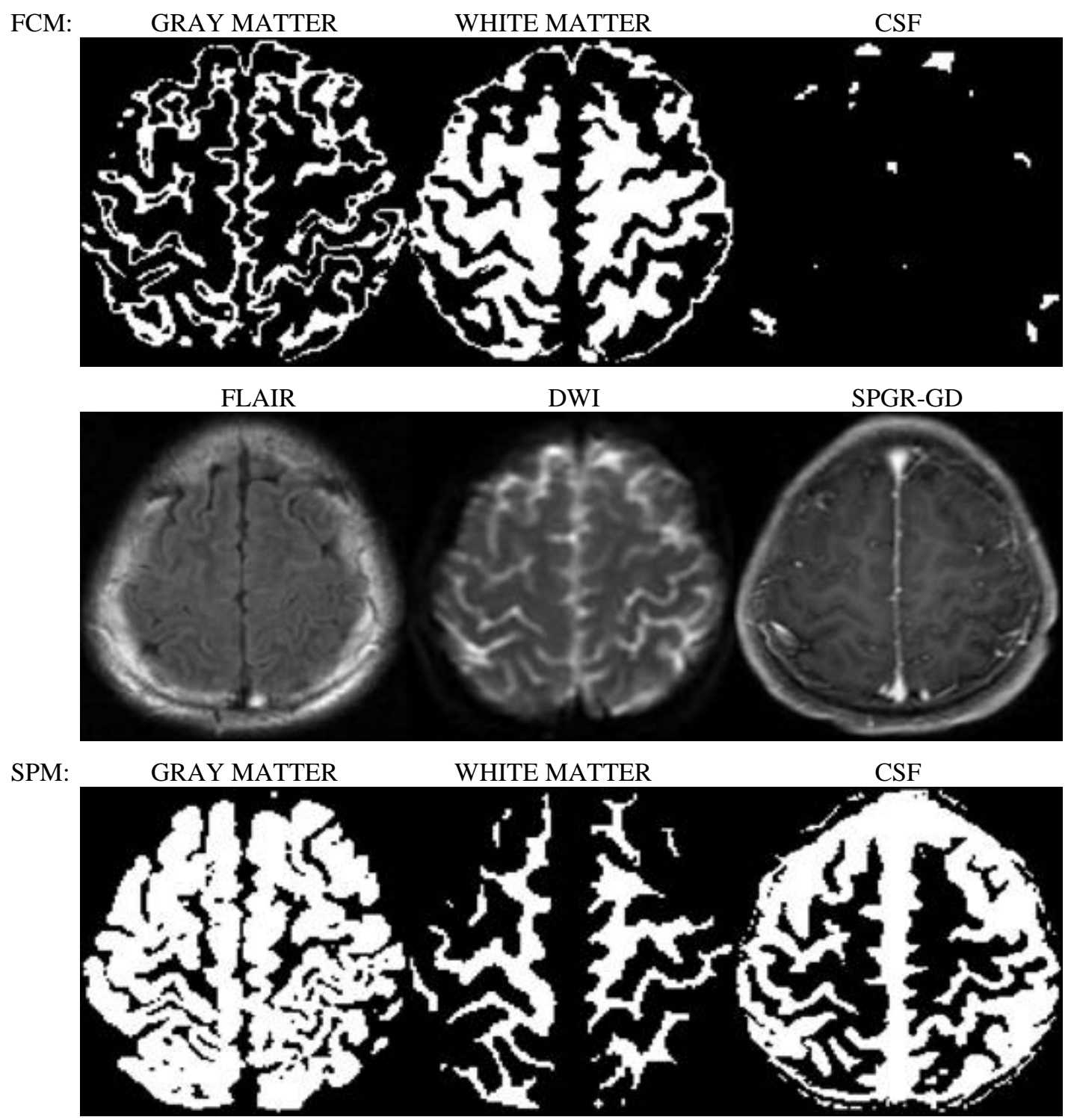

Diff. image: GRAY MATTER

WHITE MATTER

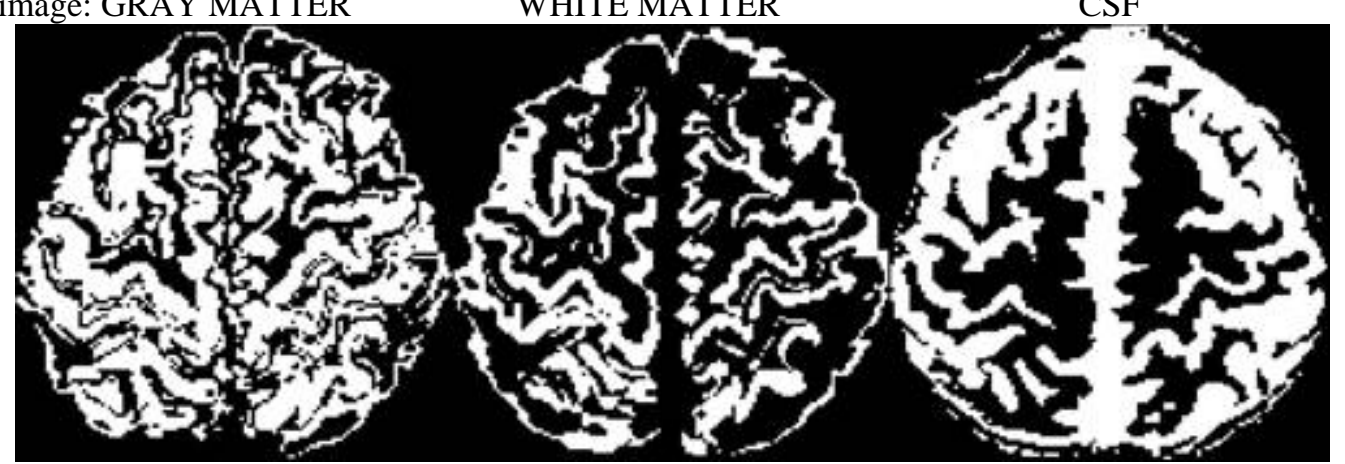

Figure A-37 Comparison of FCM and SPM segmented images using five feature vectors 
Normal subject (N3): Comparison between FCM and SPM segmented images using five feature vectors.

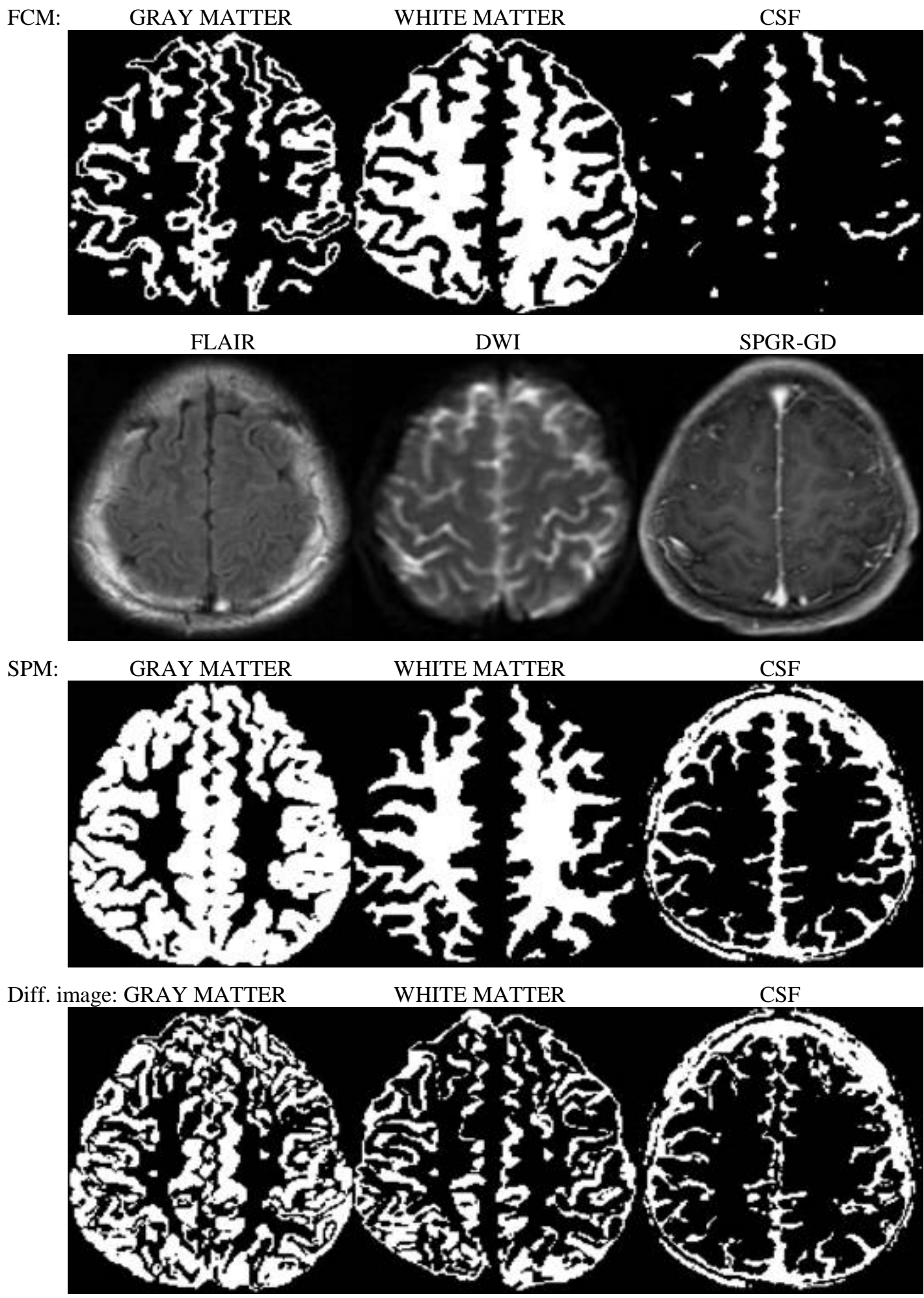

Figure A-38 Comparison of FCM and SPM segmented images using five feature vectors 
Normal subject (N4): Comparison between FCM and SPM segmented images using five feature vectors.

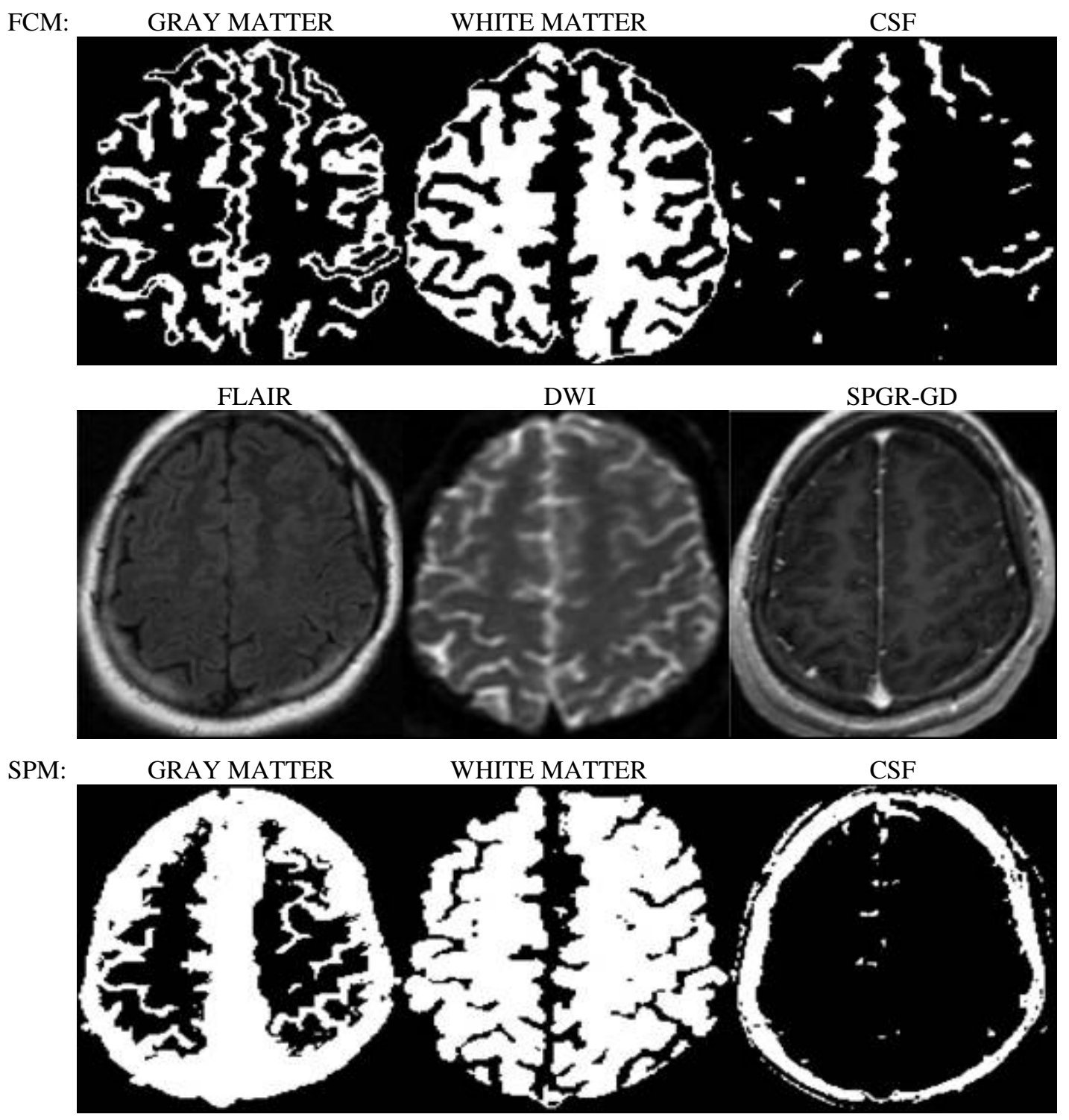

Diff. image: GRAY MATTER

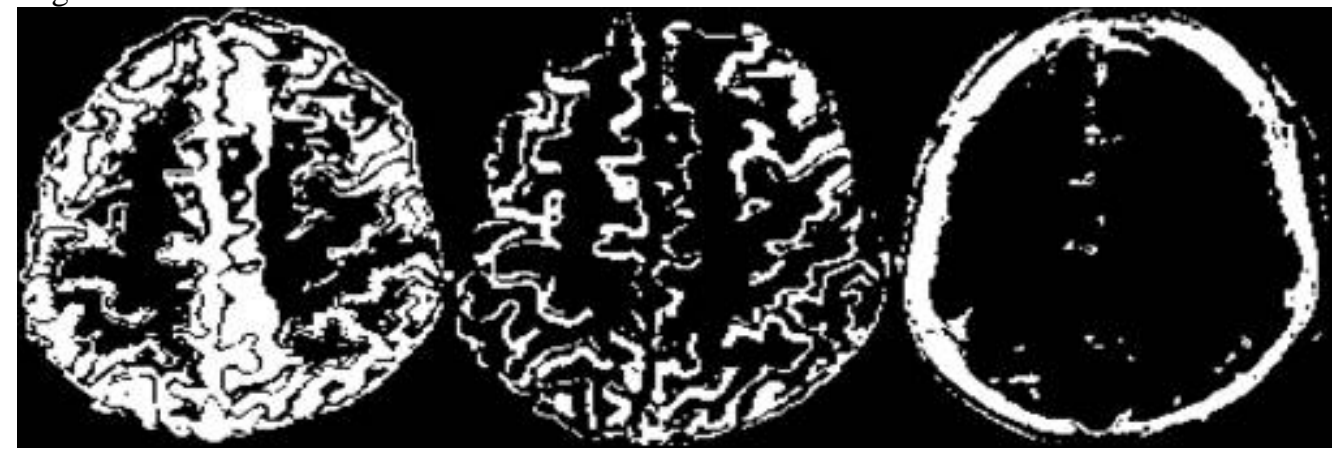

Figure A-39 Comparison of FCM and SPM segmented images using five feature vectors 
Tumor patient (P1): Comparison between FCM and SPM segmented images using five feature vectors.

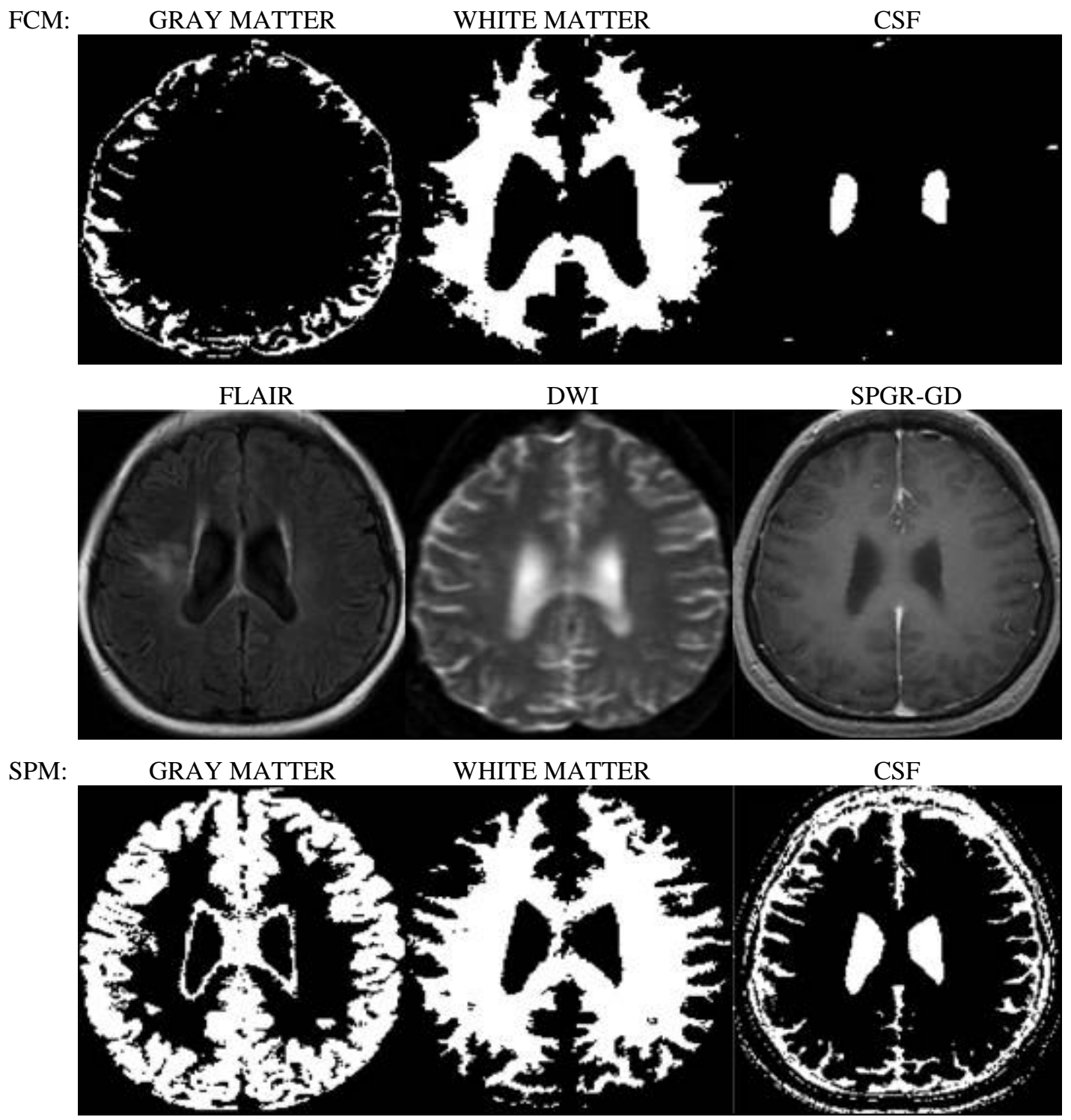

Diff image: GRAY MATTER WHITE MATTER CSF

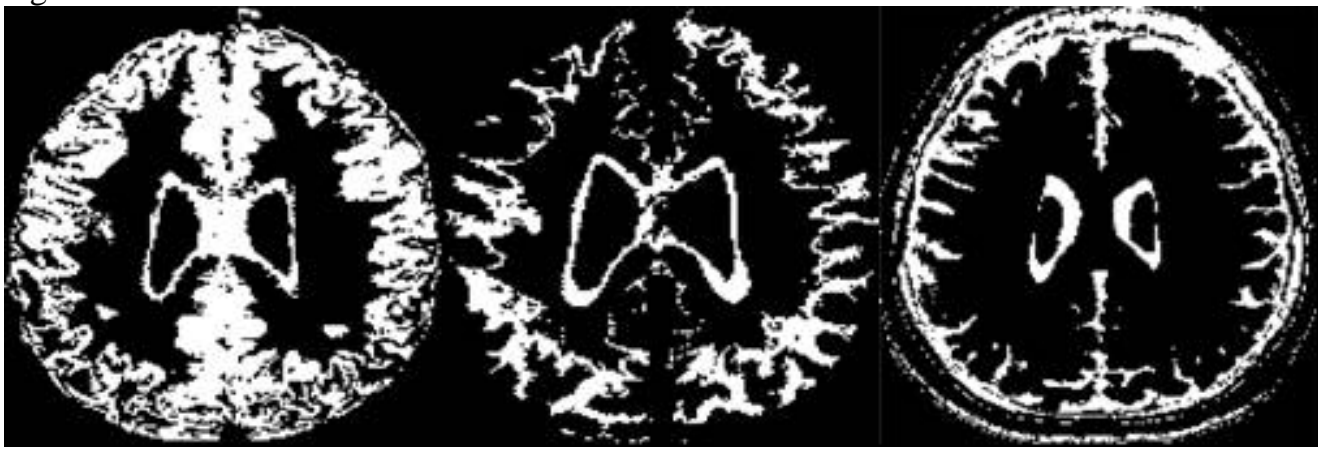

Figure A-40 Comparison of FCM and SPM segmented images using five feature vectors 
Tumor patient (P2): Comparison between FCM and SPM segmented images using five feature vectors.

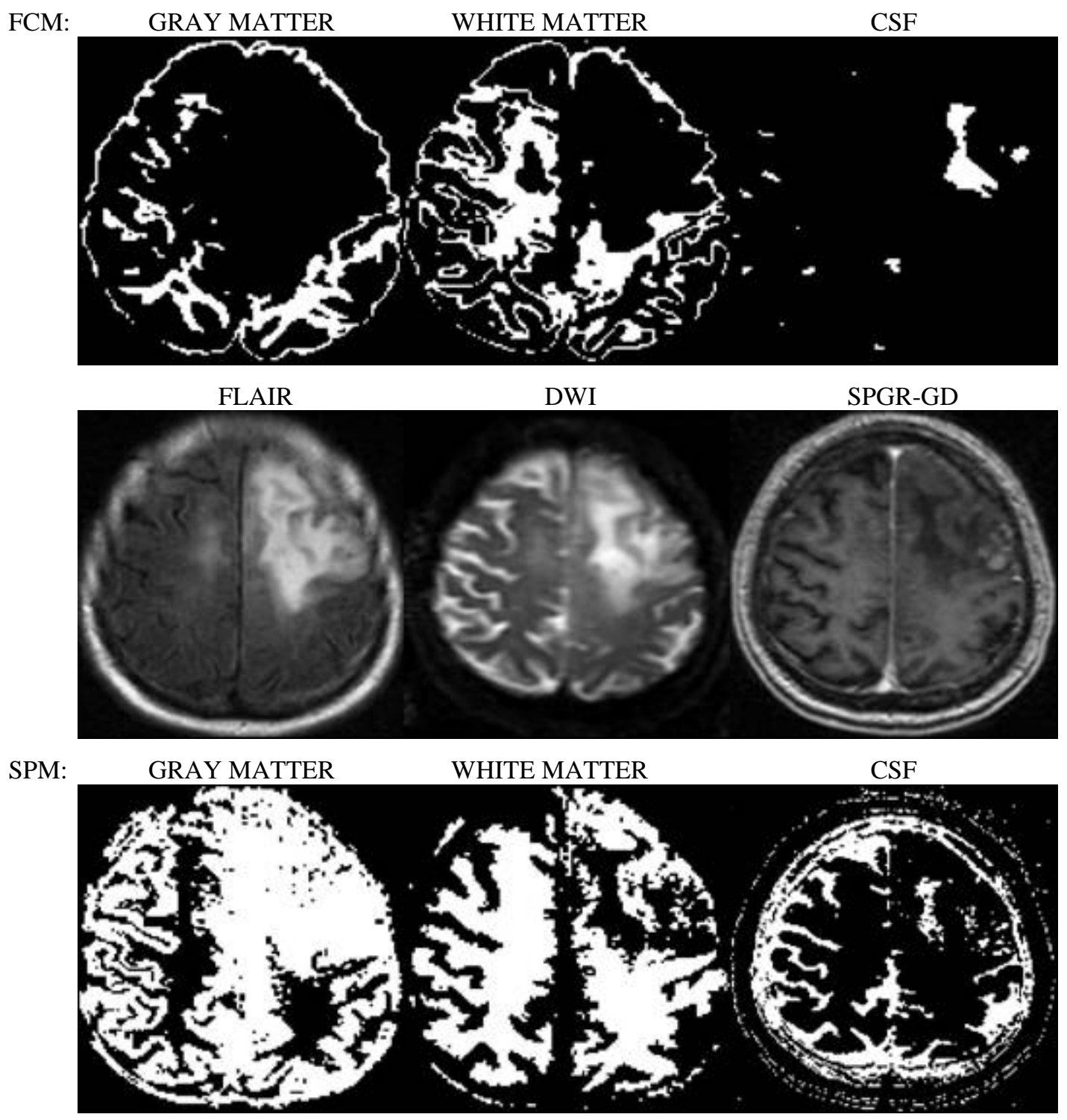

Diff image: GRAY MATTER WHITE MATTER CSF

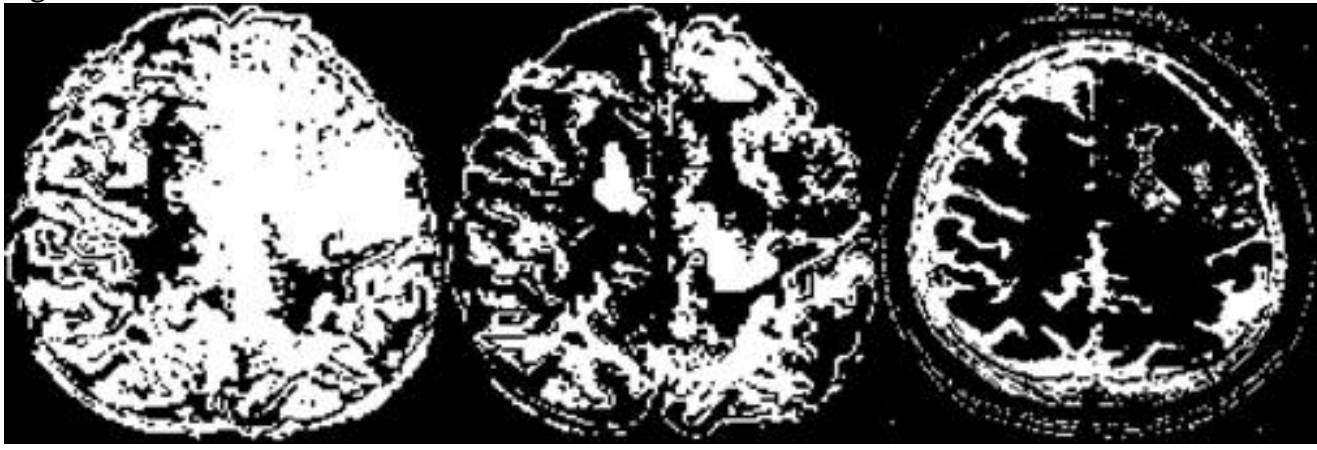

Figure A-41 Comparison of FCM and SPM segmented images using five feature vectors 
Tumor patient (P3): Comparison between FCM and SPM segmented images using five feature vectors.
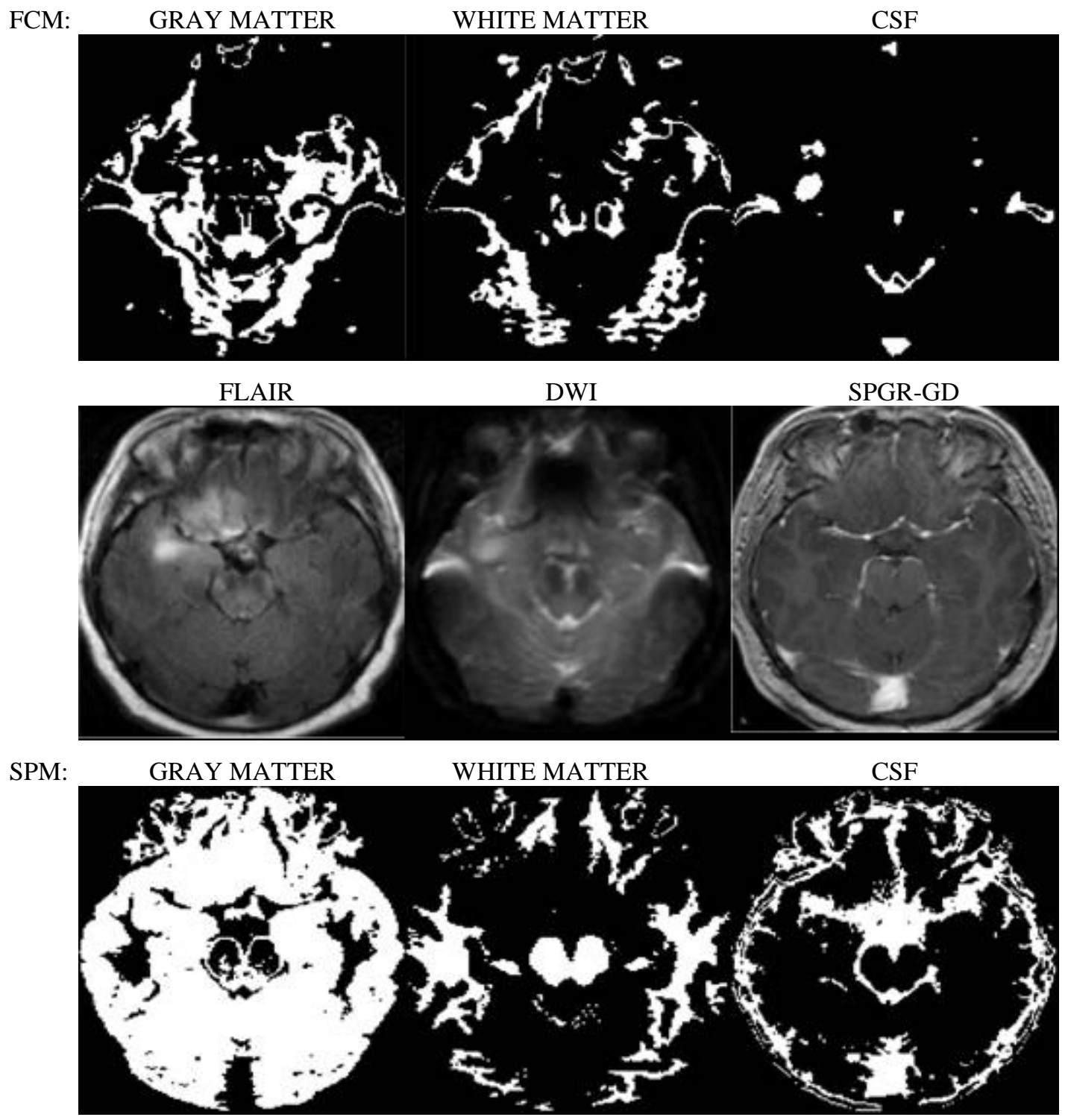

Diff image: GRAY MATTER

WHITE MATTER

CSF

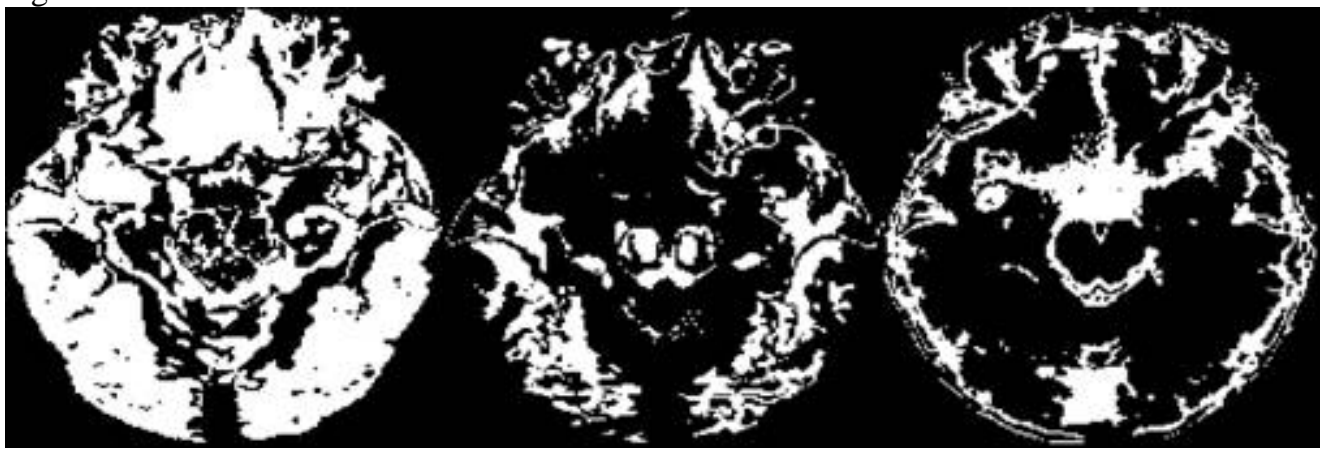

Figure A-42: Comparison of FCM and SPM segmented images using five feature vectors. 